\title{
Absolutely Continuous Spectrum for Quantum Trees
}

\author{
Nalini Anantharaman ${ }^{1}$, Maxime Ingremeau ${ }^{2}$, Mostafa Sabri $^{3}$, Brian Winn $^{4}$ (D) \\ ${ }^{1}$ Université de Strasbourg, CNRS, IRMA UMR 7501, 67000 Strasbourg, France. \\ E-mail: anantharaman@math.unistra.fr \\ ${ }^{2}$ Laboratoire J.A.Dieudonné, UMR CNRS-UNS 7351, Université Côte d'Azur, 06108 Nice, France. \\ E-mail: maxime.ingremeau@univ-cotedazur.fr \\ 3 Department of Mathematics, Faculty of Science, Cairo University, Giza 12613, Egypt. \\ E-mail: mmsabri@ sci.cu.edu.eg \\ 4 Department of Mathematical Sciences, Loughborough University, Leicestershire LE11 3TU, UK. \\ E-mail: b.winn@lboro.ac.uk
}

Received: 2 April 2020 / Accepted: 15 January 2021

Published online: 18 February 2021 - (C) The Author(s) 2021

\begin{abstract}
We study the spectra of quantum trees of finite cone type. These are quantum graphs whose geometry has a certain homogeneity, and which carry a finite set of edge lengths, coupling constants and potentials on the edges. We show the spectrum consists of bands of purely absolutely continuous spectrum, along with a discrete set of eigenvalues. Afterwards, we study random perturbations of such trees, at the level of edge length and coupling, and prove the stability of pure AC spectrum, along with resolvent estimates.
\end{abstract}

\section{Introduction}

Our aim in this paper is to establish the existence of bands of purely absolutely continuous (AC) spectrum for a large family of quantum trees. One of our motivations is to provide a collection of examples relevant for the Quantum Ergodicity result proven in [6].

For discrete trees, the problem is quite well understood when the tree is somehow homogeneous. The adjacency matrix of the $(q+1)$-regular tree $\mathbb{T}_{q}$ has pure AC spectrum $[-2 \sqrt{q}, 2 \sqrt{q}]$ as is well-known [21]. If we fix a root $o \in \mathbb{T}_{q}$ and regard the tree as descending from $o$, then the subtree descending from any offspring is the same (each is a $q$-ary tree), except for the subtree at the origin (which has $(q+1)$ children). We say that $\mathbb{T}_{q}$ has two "cone types". It was shown in [19] that if $\mathbb{T}$ is a general tree with finitely many cone types, such that each vertex has a child of its own type, and all types arise in each progeny subtree, then the spectrum consists of bands of pure AC spectrum. This problem was revisited in [5], where these assumptions were relaxed to allow $\mathbb{T}$ to be any universal cover of a finite graph of minimal degree at least 2 (see also [9] for similar results concerning more general operators on these discrete trees). In this case however, besides the bands of $\mathrm{AC}$ spectrum, a finite number of eigenvalues may appear.

A natural question is whether AC spectrum survives if we add a potential. This is motivated by the famous Anderson model [8] where random independent, identicallydistributed potentials are attached at lattice sites. It remains a major open problem to prove such stability for the Anderson model on the Euclidean lattice $\mathbb{Z}^{d}, d \geq 3$ [30]. 
The first mathematical proof showing the stability of pure AC spectrum was obtained in [22] in the case of regular trees (Bethe lattice) under weak random perturbations, thus providing the first example of spectral delocalization for an Anderson model. More general trees were subsequently treated in $[16,20]$, always in the setting of discrete Schrödinger operators. The stability of AC spectrum under perturbation by a non-random radial potential was proved in [19] in case of non-regular trees of finite cone type.

In this article we consider quantum trees, i.e. each edge is endowed some length $L_{e}$ and we study differential operators acting on the edges with appropriate boundary conditions at the vertices specified by certain coupling constants. The presence of AC spectrum for quantum trees appears to have been studied less systematically than in the case of discrete Schrödinger operators. In case of regular trees $\mathbb{T}_{q}$, it was shown in [12] that the quantum tree obtained by endowing each edge with the same length $L$, the same symmetric potential $W$ on the edges and the same coupling constant $\alpha$ at the vertices, has a spectrum consisting of bands of pure AC spectrum, along with eigenvalues between the bands. The setting was a bit generalized quite recently in [13], where each vertex in a $2 q$ regular tree is surrounded by the same set of lengths $\left(L_{1}, \ldots, L_{q}\right)$, each length repeated twice, similarly the same set of symmetric potentials $\left(W_{1}, \ldots, W_{q}\right)$, and the boundary conditions are taken to be Kirchhoff. The nature of the spectrum is partly addressed, but the possibility that it consists of a discrete set of points is not excluded. Finally, it was shown in [1] that the AC spectrum of the equilateral quantum tree [12] remains stable under weak random perturbation of the edge lengths. The theorem however does not yield purity of the AC spectrum in some interval; one can only infer that the Lebesgue measure of the perturbed $\mathrm{AC}$ spectrum is close to the unperturbed one. We also mention the papers $[17,28]$ which consider radial quantum trees, for which a reduction to a half-line model can be performed.

Our aim here is twofold. First, go beyond regular graphs. We are mainly interested in the case where the tree is the universal cover of some compact quantum graph. This implies the set of different lengths, potentials and coupling constants is finite, but the situation can be much more general than the special Cayley graph setting considered in [13]. We show in this framework that the spectrum will consist of (nontrivial) bands of pure AC spectrum, plus some discrete set of eigenvalues. Next, we consider random perturbations of these trees. We can perturb both the edge lengths and coupling constants. This setting is more general than [1], where the tree was regular and the coupling constants were zero. But our main motivation here is especially to derive the purity of the perturbed AC spectrum, along with a strong control on the resolvent, which is an important ingredient to prove quantum ergodicity for large quantum graphs. We do this in a companion paper [6].

\subsection{Some definitions.}

1.1.1. Quantum graphs Let $G=(V, E)$ be a graph with vertex set $V$ and edge set $E$. We will assume that there are no self-loops and that there is at most one edge between any two vertices, so that we can see $E$ as a subset of $V \times V$. For each vertex $v \in V$, we denote by $d(v)$ the degree of $v$. We let $B=B(G)$ be the set of oriented edges (or bonds), so that $|B|=2|E|$. If $b \in B$, we shall denote by $\hat{b}$ the reverse bond. We write $o_{b}$ for the origin of $b$ and $t_{b}$ for the terminus of $b$. We define the map $e: B \longrightarrow E$ by $e\left(\left(v, v^{\prime}\right)\right)=\left\{v, v^{\prime}\right\}$. An orientation of $G$ is a map or $: E \longrightarrow B$ such that $e \circ$ or $=I d_{E}$.

A length graph $(V, E, L)$ is a connected combinatorial graph $(V, E)$ endowed with a map $L: E \rightarrow(0, \infty)$. If $b \in B$, we denote $L_{b}:=L(e(b))$. 
A quantum graph $\mathbf{Q}=(V, E, L, W, \alpha)$ is the data of:

- A length graph $(V, E, L)$,

- A potential $W=\left(W_{b}\right)_{b \in B} \in \bigoplus_{b \in B} C^{0}\left(\left[0, L_{b}\right] ; \mathbb{R}\right)$ satisfying for $x \in\left[0, L_{b}\right]$,

$$
W_{\widehat{b}}\left(L_{b}-x\right)=W_{b}(x) .
$$

- Coupling constants $\alpha=\left(\alpha_{v}\right)_{v \in V} \in \mathbb{R}^{V}$.

The underlying metric graph is the quotient

$$
\mathscr{G}:=\left\{x=\left(b, x_{b}\right) ; b \in B, x_{b} \in\left[0, L_{b}\right]\right\} / \sim,
$$

where $\left(b, x_{b}\right) \sim\left(b^{\prime}, x_{b^{\prime}}^{\prime}\right)$ if $b^{\prime}=\hat{b}$ and $x_{b^{\prime}}^{\prime}=L_{b}-x_{b}$.

A function on the graph will be a map $f: \mathscr{G} \longrightarrow \mathbb{R}$. It can also be identified with a collection of maps $\left(f_{b}\right)_{b \in B}$ such that $f_{b}\left(L_{b}-\cdot\right)=f_{\hat{b}}(\cdot)$. We say that $f$ is supported on $e$ for some $e \in E$ if $f_{b} \equiv 0$ unless $e(b)=e$.

If each $f_{b}$ is positive and measurable, we define $\int_{\mathscr{G}} f(x) \mathrm{d} x:=\frac{1}{2} \sum_{b \in B} \int_{0}^{L_{b}} f_{b}\left(x_{b}\right) \mathrm{d} x_{b}$. We may then define the spaces $L^{p}(\mathscr{G})$ for $p \in[1, \infty]$ in the usual way. tion).

Condition (1.1) simply requires $W$ to be well-defined on $\mathscr{G}$ (no symmetry assump-

When $G=(V, E)$ is a tree, i.e., contains no cycles (which will be the case in most of the paper), we say that $\mathbf{Q}$ is a quantum tree, and we denote it by the letter $\mathbf{T}$ rather than $\mathbf{Q}$, while the set $\mathscr{G}$ is called a metric tree and is denoted by $\mathscr{T}$.

1.1.2. Orienting quantum trees Let $\mathbb{T}$ be a combinatorial tree, that is, a graph containing no cycles. We denote its vertex set by $V(\mathbb{T})$ or just $V$, its edge set by $E(\mathbb{T})$, and its set of oriented edges by $B(\mathbb{T})$. In all the paper, we will often write $v \in \mathbb{T}$ instead of $v \in V(\mathbb{T})$ to lighten the notations.

In this paragraph, we explain how we can present the tree $\mathbb{T}$ in a coherent view, that is to say, fix an oriented edge $b_{o} \in B(\mathbb{T})$, and give an orientation to all the other edges of $\mathbb{T}$, by asking that they "point in the same direction as $b_{o}$ ".

More precisely, let us fix once and for all an oriented edge $b_{o} \in B(\mathbb{T})$, corresponding to an edge $e_{o} \in E(\mathbb{T})$. If we remove the edge $e_{o}$ from $\mathbb{T}$, we obtain two connected components which are still combinatorial trees. We will write $\mathbb{T}_{b_{o}}^{+}$for the connected component containing $t_{b_{o}}$, and $\mathbb{T}_{b_{o}}^{-}$for the component containing $o_{b_{o}}$.

Let $v \in \mathbb{T}_{b_{o}}^{+}$be at a distance $n$ from $t_{b_{o}}$. Amongst the neighbours of $v$, one of them is at distance $n-1$ from $t_{b_{o}}$ : we denote it by $v_{-}$, and say that $v_{-}$is the parent of $v$. The other neighbours of $v$ are at a distance $n+1$ from $t_{b_{o}}$, and are called the children of $v$. The set of children of $v$ is denoted by $\mathscr{N}_{v}^{+}$. On the contrary, if $v \in \mathbb{T}_{b_{o}}^{-}$is at distance $n$ from $o_{b_{o}}$, its unique neighbour at a distance $n-1$ from $o_{b_{o}}$ is called the child of $v$, and denoted by $v_{+}$, and its other neighbours are its parents, whose set we denote by $\mathscr{N}_{v}^{-}$. These definitions are natural if we see the tree at the left of Fig. 1 as a genealogical tree.

Let $V^{*}=\left(V(\mathbb{T}) \backslash\left\{o_{b_{o}}, t_{b_{o}}\right\}\right) \cup\{o\}$. We define a map $\mathfrak{b}: V^{*} \longrightarrow B(\mathbb{T})$ as follows: we set $\mathfrak{b}(o)=b_{o}$, and, if $v \in \mathbb{T}_{b_{o}}^{+}$, then $\mathfrak{b}(v)=\left(v_{-}, v\right)$, while if $v \in \mathbb{T}_{b_{o}}^{-}$, then $\mathfrak{b}(v)=\left(v, v_{+}\right)$. One easily sees that $e \circ \mathfrak{b}: V^{*} \longrightarrow E(\mathbb{T})$ is a bijection, so that $\mathfrak{b} \circ(e \circ \mathfrak{b})^{-1}$ is an orientation of $\mathbb{T}$. The map $\mathfrak{b}$ serves to index all oriented edges: those in $\mathbb{T}_{b_{o}}^{+}$by their terminus, those in $\mathbb{T}_{b_{o}}^{-}$by their origin, and $b_{o}$ by its "midpoint" $o$. The 
latter makes sense once we turn $\mathbb{T}$ into a quantum tree $\mathbf{T}$. We denote $L_{v}:=L_{\mathfrak{b}(v)}$ and $W_{v}:=W_{\mathfrak{b}(v)}$. The metric tree $\mathscr{T}$ can be identified with the set

$$
\mathscr{T} \equiv \bigsqcup_{v \in V^{*}}\left[0, L_{v}\right]=\left\{x=\left(v, x_{v}\right) \mid v \in V^{*}, x_{v} \in\left[0, L_{v}\right]\right\} .
$$

A function on $\mathscr{T}$ will then be the data of $\psi=\left(\psi_{v}\right)_{v \in V^{*}}$, where each $\psi_{v}$ is a function of the variable $x_{v} \in\left[0, L_{v}\right]$.

On a quantum tree, we consider the Schrödinger operator

$$
\left(\mathscr{H}_{\mathbf{T}} \psi_{v}\right)\left(x_{v}\right)=-\psi_{v}^{\prime \prime}\left(x_{v}\right)+W_{v}\left(x_{v}\right) \psi_{v}\left(x_{v}\right)
$$

with domain $D\left(\mathscr{H}_{\mathbf{T}}\right)$, the set of functions $\left(\psi_{v}\right) \in \bigoplus_{v \in V^{*}} W^{2,2}\left(0, L_{v}\right)$ satisfying the socalled $\delta$-conditions. Namely, for all $v \in \mathbb{T}_{b_{o}}^{+}$,

$$
\psi_{v}\left(L_{v}\right)=\psi_{v_{+}}(0) \forall v_{+} \in \mathscr{N}_{v}^{+} \quad \text { and } \quad \sum_{v_{+} \in \mathscr{N}_{v}^{+}} \psi_{v_{+}}^{\prime}(0)=\psi_{v}^{\prime}\left(L_{v}\right)+\alpha_{v} \psi_{v}\left(L_{v}\right) \text {, }
$$

while for all $v \in \mathbb{T}_{b_{o}}^{-}$,

$$
\psi_{v_{-}}\left(L_{v_{-}}\right)=\psi_{v}(0) \forall v_{-} \in \mathscr{N}_{v}^{-} \quad \text { and } \quad \sum_{v_{-} \in \mathscr{N}_{v}^{-}} \psi_{v-}^{\prime}\left(L_{v_{-}}\right)+\alpha_{v} \psi_{v}(0)=\psi_{v}^{\prime}(0) \text {. }
$$

Finally, we have $\psi_{o}\left(L_{o}\right)=\psi_{o_{+}}(0) \forall o_{+} \in \mathscr{N}_{o}^{+}, \sum_{o_{+}} \psi_{o_{+}}^{\prime}(0)=\psi_{o}^{\prime}\left(L_{o}\right)+\alpha_{t_{b_{o}}} \psi_{o}\left(L_{o}\right)$, and $\psi_{o_{-}}\left(L_{o_{-}}\right)=\psi_{o}(0), \sum_{o_{-}} \psi_{o_{-}}^{\prime}\left(L_{o_{-}}\right)+\alpha_{o_{b_{o}}} \psi_{o}(0)=\psi_{o}^{\prime}(0)$. In a common convention we will refer to the $\alpha_{v}=0$ case as the Kirchhoff-Neumann condition.

Remark 1.1. The above conventions mean that we see $\mathbb{T}$ as a doubly infinite genealogical tree. This is what we called the coherent view; it can also be pictured by saying that we imagine an electric flow moving from $\mathbb{T}_{b_{o}}^{-}$to $\mathbb{T}_{b_{o}}^{+}$.

There is another way of orienting the graph which we call the twisted view. This is done by turning $b_{o}$ into a V-shape and viewing $V(\mathbb{T})$ as offspring of $o$. See Fig. 1 for an illustration; here one should think that $o$ is a source from which the electric flows moves outwards. When necessary to highlight this genealogical structure, we will write $\mathbb{T}_{o}^{+}$for the set of offsprings of $o$. Each vertex $v$ has a single parent $v_{-}$and several children; all the edges take the form $\left\{v, v_{-}\right\}$for a unique $v$.

The link between the two views is immediate: functions on $\mathbb{T}_{b_{o}}^{+}$in both views coincide, while on $\mathbb{T}_{b_{o}}^{-}$, one replaces $b$ by $\hat{b}$ and derivatives take a sign. Here $\hat{b}=\left(t_{b}, o_{b}\right)$ is the edge reversal of $b$. Hence, in the twisted view, ${ }^{1}$ all functions in the domain of $\mathscr{H}$ satisfy (1.4).

1 These views of the tree have the advantage of avoiding the assumption that $\mathbb{T}$ has a special "root" vertex of degree one [1,28]. Such assumption simplifies the orientation a bit, but is not satisfied in many natural situations. As will be clear later, we will only need to study functions supported in $\mathbf{T}_{b_{o}}^{ \pm}$, which is why we did not specify what happens on $b_{o}$. But one could specify that $\psi_{o}$ from the coherent view becomes $\left(\psi_{o}^{(1)}, \psi_{o}^{(2)}\right)$ in the twisted view, with $\psi_{o}^{(1)}(x)=\psi_{o}\left(x+\frac{L_{o}}{2}\right)$ and $\psi_{o}^{(2)}(x)=\psi_{o}\left(\frac{L_{o}}{2}-x\right)$, for $x \in\left[0, \frac{L_{o}}{2}\right]$. 


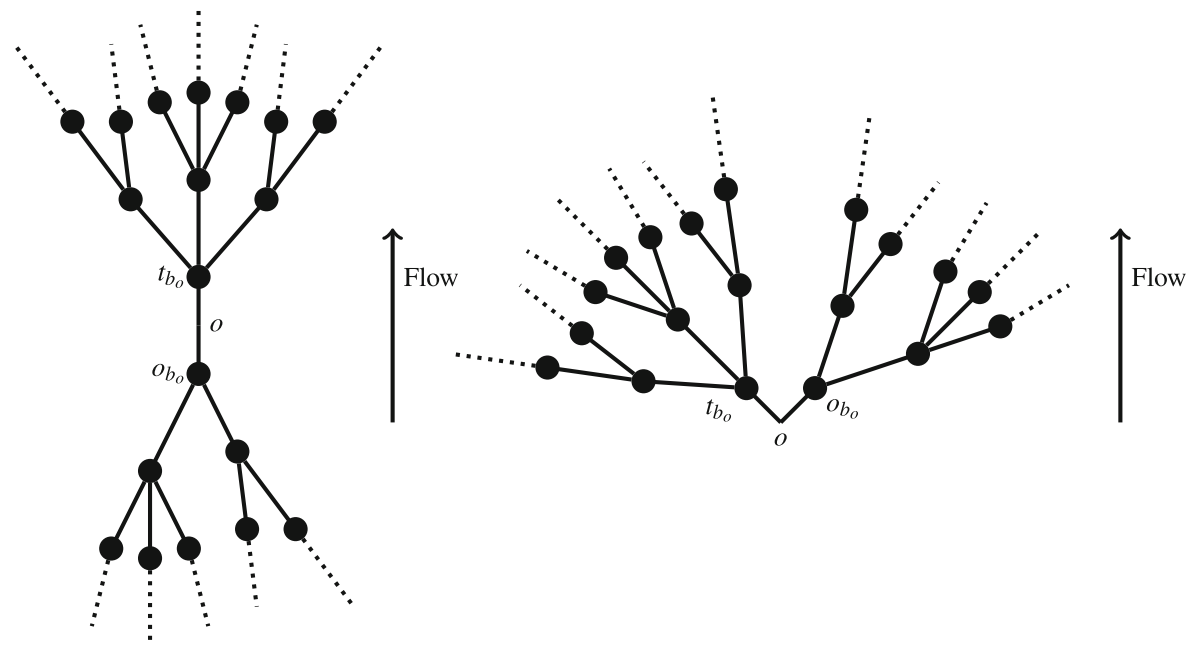

Coherent view

Twisted view

Fig. 1. The two views of a tree

Given $v \in V^{*}, z \in \mathbb{C}$, let $C_{z}(x)$ and $S_{z}(x)$ be a basis of solutions of the problem $-\psi_{v}^{\prime \prime}+W_{v} \psi_{v}=z \psi_{v}$ satisfying

$$
\left(\begin{array}{ll}
C_{z}(0) & S_{z}(0) \\
C_{z}^{\prime}(0) & S_{z}^{\prime}(0)
\end{array}\right)=\left(\begin{array}{ll}
1 & 0 \\
0 & 1
\end{array}\right)
$$

Then any solution $\psi_{v}$ of the problem satisfies

$$
\left(\begin{array}{l}
\psi_{v}\left(L_{v}, z\right) \\
\psi_{v}^{\prime}\left(L_{v}, z\right)
\end{array}\right)=M_{z}(v)\left(\begin{array}{l}
\psi_{v}(0, z) \\
\psi_{v}^{\prime}(0, z)
\end{array}\right) \quad \text { where } M_{z}(v)=\left(\begin{array}{ll}
C_{z}\left(L_{v}\right) & S_{z}\left(L_{v}\right) \\
C_{z}^{\prime}\left(L_{v}\right) & S_{z}^{\prime}\left(L_{v}\right)
\end{array}\right)
$$

If $W_{v} \equiv 0$ then the basis of solutions is

$$
S_{z}(x)=\frac{\sin \sqrt{z} x}{\sqrt{z}}, \quad C_{z}(x)=\cos \sqrt{z} x
$$

if $W_{v}(x)=c_{1}+c_{2} \cos \left(2 \pi x / L_{v}\right)$ then $S_{z}, C_{z}$ would be Mathieu functions. It is a standard fact that $S_{z}(x), C_{z}(x)$ are analytic functions of $z \in \mathbb{C}$ (see for instance [26, Chapter 1]).

1.2. Trees of finite cone type. We define a cone in $\mathbb{T}$ to be a subtree of the form $\mathbb{T}_{b}^{+}$or $\mathbb{T}_{b}^{-}$, for some $b \in B(\mathbb{T})$. Each cone $\mathbb{T}_{b}^{+}$has an origin $t_{b}$, and each cone $\mathbb{T}_{b}^{-}$has an end $o_{b}$.

We say that two quantum cones $\mathbf{T}_{b}^{+}$and $\mathbf{T}_{b^{\prime}}^{+}$are isomorphic if there is an isomorphism of combinatorial graphs $\varphi: \mathbb{T}_{b}^{+} \rightarrow \mathbb{T}_{b^{\prime}}^{+}$such that $L_{\varphi(v)}=L_{v}, W_{\varphi(v)}=W_{v}$ and $\alpha_{\varphi(v)}=\alpha_{v}$ for all $v \in \mathbb{T}_{b}^{+}$. Isomorphic $\mathbf{T}_{b}^{-}$and $\mathbf{T}_{b^{\prime}}^{-}$are defined the same way.

We say that $\mathbf{T}$ is a tree of finite cone type if there exists $b_{o} \in B(\mathbb{T})$ such that:

(i) There are finitely many non-isomorphic quantum cones $\mathbf{T}_{\left(v_{-}, v\right)}^{+}$as $v \in \mathbb{T}_{b_{o}}^{+}$. 
(ii) There are finitely many non-isomorphic quantum cones $\mathbf{T}_{\left(w, w_{+}\right)}^{-}$as $w \in \mathbb{T}_{b_{o}}^{-}$.

Here $\left(t_{b_{o}}\right)_{-}=o_{b_{o}}$ and $\left(o_{b_{o}}\right)_{+}=t_{b_{o}}$. Note that in a regular tree, all cones $\mathbb{T}_{b}^{ \pm}$are isomorphic, but a necessary condition for it to be a quantum tree of finite cone type, is that its edges and vertices be endowed with finitely many lengths, potentials and coupling constants. $^{2}$

If $\mathbf{T}$ is a tree of finite cone type, with $b_{o} \in B(\mathbb{T})$ fixed, we may introduce a type function $\ell: \mathbb{T}_{b_{o}}^{+} \rightarrow \mathbb{N}_{0}=\mathbb{N} \cup\{0\}$, taking values in a finite set, such that $\ell(v)=\ell(w)$ iff $\mathbf{T}_{\left(v_{-}, v\right)}^{+} \equiv \mathbf{T}_{\left(w_{-}, w\right)}^{+}$as quantum trees. Similarly, $\ell: \mathbb{T}_{b_{o}}^{-} \rightarrow \mathbb{N}_{0}$ satisfies $\ell(v)=\ell(w)$ iff $\mathbf{T}_{\left(v, v_{+}\right)}^{-} \equiv \mathbf{T}_{\left(w, w_{+}\right)}^{-}$. Note that if $\ell(v)=\ell(w)$, then $W_{v}=W_{w}, L_{v}=L_{w}$ and $\alpha_{v}=\alpha_{w}$, since the corresponding isomorphism respects this information. Hence, any coherent quantum tree $\mathbf{T}$ of finite cone type comes with the following structure:

(a) A fixed $b_{o} \in B(\mathbb{T})$.

(b) Two finite sets of labels $\mathfrak{A}^{+}=\left\{i_{1}, \ldots, i_{m}\right\}, \mathfrak{A}^{-}=\left\{j_{1}, \ldots, j_{n}\right\}$ and two matrices $M=\left(M_{i, j}\right)_{i, j \in \mathfrak{A}^{+}}, N=\left(N_{i, j}\right)_{i, j \in \mathfrak{A}^{-}}$. If $v \in \mathbb{T}_{b_{o}}^{+}$has type $j$, it has $M_{j, k}$ children of type $k$. If $w \in \mathbb{T}_{b_{o}}^{-}$has type $j$, it has $N_{j, k}$ parents of type $k$.

(c) Finite sets $\left\{L_{i}\right\}_{i \in \mathfrak{A}^{ \pm}},\left\{W_{i}\right\}_{i \in \mathfrak{A}^{ \pm}}$and $\left\{\alpha_{i}\right\}_{i \in \mathfrak{A}^{ \pm}}$encoding the lengths, potentials and coupling constants, respectively. More precisely, $b_{o}$ is endowed a special length $L_{o}$ and potential $W_{o}$. If $\left(v_{-}, v\right) \in \mathbb{T}_{b_{o}}^{+}$with $\ell(v)=i$, then $L_{v}=L_{i}, W_{v}=W_{i}$ and $\alpha_{v}=\alpha_{i}$. The same attribution is made if $\left(v, v_{+}\right) \in \mathbb{T}_{b_{o}}^{-}$with $\ell(v)=i$.

If we take the twisted view instead, we only need one alphabet $\mathfrak{A}=\mathfrak{A}^{+} \cup \mathfrak{A}^{-}$and

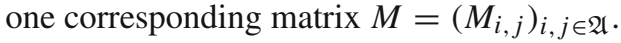

A trivial example is the equilateral, $(q+1)$-regular quantum tree, with identical symmetric potentials $W$ on each edge and identical coupling constant $\alpha$ on each vertex [12]. In this case, all vertices in $\mathbb{T}_{b_{o}}^{ \pm}$have the same type, and we get two $1 \times 1$ matrices $M=N=(q)$.

An important class of examples comes from universal covers of finite undirected graphs. More precisely, if $G$ is a finite undirected graph and $\mathbb{T}$ is its universal cover, then $\mathbb{T}$ is a combinatorial tree satisfying condition (i). If we endow $G$ with a quantum structure $\mathbf{G}$ and lift it to $\mathbb{T}$ in the natural way, then the corresponding $\mathbf{T}$ will be a quantum tree of finite cone type.

Quantum trees of finite cone type satisfying (a)-(c) will be our basic, "unperturbed" trees. We denote the Schrödinger operator (1.2) acting in this setting as $\mathscr{H}_{0}$. It is selfadjoint, as a consequence of Theorem 17 in [23]. Later on, we shall study random perturbations of these trees, and denote the corresponding self-adjoint operator by $\mathscr{H}_{\varepsilon}{ }^{\omega}$, where $\varepsilon$ is the strength of the disorder.

We make the following assumption on $\mathbb{T}$ :

(C1*) For any $k, l \in \mathfrak{A}^{+}$, there is $n=n(k, l)$ such that $\left(M^{n}\right)_{k, l} \geq 1$. Similarly, for $i, j \in \mathfrak{A}^{-}$, there is $n=n(i, j)$ with $\left(N^{n}\right)_{i, j} \geq 1$.

See Remark 1.2 below for a discussion of this condition. We may now state a first theorem, which describes the structure of the spectrum of $\mathscr{H}_{0}=\mathscr{H}_{\mathbf{T}}$ on a tree $\mathbf{T}$ of finite cone type. We denote by $G_{0}^{z}(x, y)=\left(\mathscr{H}_{0}-z\right)^{-1}(x, y)$ the Green's function of $\mathscr{H}_{0}$.

\footnotetext{
2 Also note that it is not required that there are finitely many non-isomorphic quantum trees $\mathbf{T}_{\left(v_{-}, v\right)}^{-}$as $v \in \mathbb{T}_{b_{o}}^{+}$. To illustrate this point, consider the binary tree (so each vertex has 3 neighbors except for the special root $\star$ with 2 neighbors), let $b_{o}=(\star, v)$, with $v$ either neighbor. Then all cones $\mathbb{T}_{b}^{+} \subset \mathbb{T}_{b_{o}}^{+}$look the same; they are binary trees. However, the backward cones $\mathbb{T}_{b}^{-}$are distinct in each generation, because they "see" the special root at distinct distances. Despite this, $\mathbb{T}$ has finite cone type.
} 
Theorem 1.1. Let $M, N$ satisfy $(\boldsymbol{C 1} *)$. Then the spectrum of $\mathscr{H}_{0}$ consists of a disjoint union of closed intervals and of isolated points: $\sigma\left(\mathscr{H}_{0}\right)=\left(\bigsqcup_{r} I_{r}\right) \cup \mathfrak{P}$, where the $I_{r}$ are closed intervals, and $\mathfrak{P}$ is a discrete set. The spectrum is purely absolutely continuous in the interior of each band $\stackrel{\circ}{I}_{r}$. For $\lambda \in \stackrel{\circ}{I}_{r}$, and for any $v \in \mathbb{T}$, the limit $G_{0}^{\lambda+\mathrm{i} 0}(v, v)$ exists and satisfies $\operatorname{Im} G_{0}^{\lambda+\mathrm{i} 0}(v, v)>0$, where $G_{0}^{z}$ is the Green's function of $\mathscr{H}_{0}$.

Let $R_{z, 0}^{ \pm}$be the Weyl-Titchmarsh functions of $\mathscr{H}_{0}$ as defined in [1], see (2.2). Let $R_{\lambda, 0}^{ \pm}=R_{\lambda+\mathrm{i} 0,0}^{ \pm}$when the limit exists. Theorem 1.1 implies that $\operatorname{Im} R_{\lambda, 0}^{+}(v)+\operatorname{Im} R_{\lambda, 0}^{-}(v)>$ 0 in $\stackrel{\circ}{I}_{r}$. We will need the stronger property that $\operatorname{Im} R_{\lambda, 0}^{+}(v)>0$ for all $v$. For this, we introduce the following strengthening of $\left(\mathrm{C1}^{*}\right)$.

(C1) The quantum tree $\mathbf{T}$ is the universal cover of a finite quantum graph $\mathbf{G}$ of minimal degree $\geq 2$ which is not a cycle.

Remark 1.2. Condition (C1*) means that on $\mathbb{T}_{b_{o}}^{+}$, any cone type $l \in \mathfrak{A}^{+}$appears as offspring of any $k \in \mathfrak{A}^{+}$after a finite number of generations, and similarly for $\mathbb{T}_{b_{o}}^{-}$. It is not required that cone types in $\mathfrak{A}^{-}$appear in $\mathbb{T}_{b_{o}}^{+}$- we only need the matrices $M$ and $N$ to be separately irreducible. We can also allow for "rooted" trees where the root $o$ has degree one. In this case the situation is a bit simpler actually; we only have to deal with one matrix $M$. Condition $\left(\mathbf{C} \mathbf{1}^{*}\right)$ applies in particular to trees with a "radial periodic" data, i.e. data that are periodic functions of the distance to the origin (such as some examples appearing in [28]).

Assumption (C1) implies (C1*) (see Remark 3.2), and is in fact more restrictive. In particular, T is "unimodular", that is, all data is somehow homogeneous as we move along the tree. This excludes for example the binary tree and more generally radial periodic trees, where the root plays a special role. However, such unimodular trees are still very general, they are actually the most interesting for us, and many techniques (such as a reduction to a half-line model) fail to tackle them. Even in the very simple case where the base graph $\mathbf{G}$ is regular but the edge lengths are not equal, the lifted structure in general will be neither radial periodic, nor identical around each vertex (in contrast to [13]).

Note that the case where $\mathbf{G}$ is a cycle is already known when the couplings are zero. In this case $\mathscr{H}_{\mathbf{T}}$ is just a periodic Schrödinger operator on $\mathbb{R}$ (of period $\leq|\mathbf{G}|$ ), it is well-known that the spectrum is purely AC in this case [27, Sect. XIII.16].

Theorem 1.2. If $\mathbf{T}$ satisfies $(\mathbf{C 1})$, then the spectrum of $\mathscr{H}_{0}$ consists of a disjoint union of closed intervals and of isolated points: $\sigma\left(\mathscr{H}_{0}\right)=\left(\bigsqcup_{r} I_{r}\right) \cup \mathfrak{P}$, where the $I_{r}$ are closed intervals, and $\mathfrak{P}$ is a discrete set. The spectrum is purely absolutely continuous in the interior of each band $\stackrel{\circ}{I}_{r}$. For $\lambda \in \stackrel{\circ}{I}_{r}$, the limit $R_{\lambda+\mathrm{i} 0,0}^{+}(v)$ exists for any $v \in V$ and satisfies $\operatorname{Im} R_{\lambda, 0}^{+}(v)>0$.

Remark 1.3. In Theorems 1.1 and 1.2, it is not excluded in principle that $\bigcup_{r} I_{r}=\emptyset$, i.e. the spectrum consists of isolated points. We think this never happens for infinite quantum trees of finite cone type with the $\delta$-conditions we consider, i.e. we believe these should always have some continuous spectrum. We did not find such a result in the literature however. This is why we dedicate Sect. 4 to prove the following: if T satisfies either:

(1) Assumption (C1) and has a single data $(L, \alpha, W)$ (all edges carry the same length, coupling and symmetric potential),

(2) Or has a general data $\left(L_{e}, \alpha_{o_{e}}, W_{e}\right)_{e \in E(\mathbf{G})}$, but the finite graph $G$ is moreover Hamiltonian, 
then $\mathscr{H}_{\mathbf{T}}$ always has some continuous spectrum, i.e. $\bigcup_{r} \stackrel{\circ}{I}_{r} \neq \emptyset$. Recall that a finite graph is Hamiltonian if it has a cycle that visits each vertex exactly once. Note that as a discrete tree, $\mathbb{T}$ may cover many different graphs. We only need one of these finite graphs to be Hamiltonian. For example, we can consider any regular tree, despite the fact that some regular graphs (like the Petersen graph) are not Hamiltonian.

In particular, the Cayley tree considered in [13] can be realized as the universal cover of the complete bipartite graph $K_{2 q, 2 q}$, which is Hamiltonian. For this, use the fact that $K_{2 q, 2 q}$ has a proper $2 q$-edge-colouring and put the same length/potential on edges of the same colour. The lift of this is then a tree which has the same data around each vertex, and we may take $L_{q+j}=L_{j}, W_{q+j}=W_{j}$ to be in the setting [13]. Then our theorems imply this tree has nontrivial bands of pure AC spectrum, thus enriching the results of [13]. Again, this is just one very special application of our framework.

1.3. Random perturbations of trees of finite cone type. Fix a quantum tree $\mathbf{T}$ satisfying (C1). As explained in Remark 3.2, any such tree is a tree of finite cone type. We fix an edge $e \in E(\mathbb{T})$, and see our quantum tree in the twisted view (in which all vertices are descendent of a vertex $o$ ), so as to deal with a single alphabet $\mathfrak{A}$ and a corresponding matrix $M$. We denote the lengths and coupling constants of the unperturbed tree $\mathbf{T}$ by $\left(L_{v}^{0}\right)_{v \in V^{*}}$ and $\left(\alpha_{v}^{0}\right)_{v \in V}$. These can also be denoted $\left(L_{i}^{0}\right)_{i \in \mathfrak{A} \cup\{o\}}$ and $\left(\alpha_{i}^{0}\right)_{i \in \mathfrak{A} \text {. We assume }}$ there are no potentials on the edges and the couplings are nonnegative:

$$
W_{v}^{0} \equiv 0 \quad \text { and } \quad \alpha_{v}^{0} \geq 0
$$

We now want to analyze random perturbations of $\mathbf{T}$. For this purpose, we introduce a probability space $(\Omega, \mathscr{F}, \mathbb{P})$, a family of random variables $\omega \in \Omega \mapsto\left(L_{v}^{\omega}\right)_{v \in V^{*}}$ representing random lengths, and a family of random variables $\omega \in \Omega \mapsto\left(\alpha_{v}^{\omega}\right)_{v \in V}$ representing random coupling constants. In principle we could also consider random potentials $\omega \in \Omega \mapsto\left(W_{v}^{\omega}\right)_{v \in V^{*}}$, however here we assume there are no potentials on the edges even after perturbation. We also assume the perturbed couplings are nonnegative:

$$
W_{v}^{\omega} \equiv 0 \quad \text { and } \quad \alpha_{v}^{\omega} \geq 0
$$

We make the following assumptions on the random perturbation (see Remark 1.1 for the notation $\mathbb{T}_{o}^{+}$):

(P0) The operator $\mathscr{H}_{\varepsilon}^{\omega}$ is the Laplacian on the edges acting on $\bigoplus W^{2,2}\left(0, L_{v}^{\omega}\right)$, satisfying $\delta$-conditions with coupling constants $\left(\alpha_{v}^{\omega}\right)_{v \in \mathbb{T}}$, which are assumed to satisfy

$$
L_{v}^{\omega} \in\left[L_{v}^{0}-\varepsilon, L_{v}^{0}+\varepsilon\right] \text { and } \alpha_{v}^{\omega} \in\left[\alpha_{v}^{0}-\varepsilon, \alpha_{v}^{0}+\varepsilon\right] .
$$

(P1) For all $v, w \in \mathbb{T}_{o}^{+}$, the random variables $\left(\alpha_{v}^{\omega}, L_{v}^{\omega}\right)$ and $\left(\alpha_{w}^{\omega}, L_{w}^{\omega}\right)$ are independent if the forward trees of $v$ and $w$ do not intersect, i.e. if $\mathbb{T}_{\left(v_{-}, v\right)}^{+} \cap \mathbb{T}_{\left(w_{-}, w\right)}^{+}=\emptyset$.

(P2) For all $v, w \in \mathbb{T}_{o}^{+}$that share the same label, the restrictions of the random variables $\left(\alpha^{\omega}, L^{\omega}\right)$ to the isomorphic forward trees of $v$ and $w$ are identically distributed.

Remark 1.4. Assumptions (P1) and (P2) hold, in particular, for independent identically distributed random variables (which is the main case we have in mind). 
We shall consider intervals $I$ lying in the interior of the unperturbed AC spectrum:

$$
\Sigma=\bigcup_{r} \stackrel{\circ}{I}_{r}
$$

where $I_{r}$ are given in Theorem 1.2.

We will also need to ensure that the various $\sin \left(\sqrt{\lambda} L_{v}\right)$ do not vanish. More precisely, by (P0), the perturbed lengths all lie in $\bigcup_{j \in \mathfrak{A} \cup\{0\}}\left[L_{j, \min }(\varepsilon), L_{j, \max }(\varepsilon)\right]$, where $L_{j, \min }(\varepsilon)=L_{j}^{0}-\varepsilon$ and $L_{j, \max }(\varepsilon)=L_{j}^{0}+\varepsilon$. We then assume

$$
I \cap \mathscr{D}=\varnothing,
$$

where the set $\mathscr{D}=\mathscr{D}_{\varepsilon}$ is a "thickening" of the Dirichlet spectrum, given by

$$
\mathscr{D}=\bigcup_{j \in \mathfrak{A} \cup\{o\}} \bigcup_{n \geq 0}\left[\frac{\pi^{2} n^{2}}{L_{j, \max }^{2}(\varepsilon)}, \frac{\pi^{2} n^{2}}{L_{j, \min }^{2}(\varepsilon)}\right] .
$$

This ensures that $\sin \left(\sqrt{\lambda} L_{v}^{\omega}\right), \sin \left(\sqrt{\lambda} L_{v}^{0}\right) \neq 0$ for any $\lambda \in I, v \in \mathbb{T}$ and $\omega$.

Recall that the Weyl-Titchmarsh functions $R_{z}^{+}(v)$ will be introduced in (2.2). Introduce the following condition:

(Green-s) There is a non-empty open set $I_{1}$ and some $s>0$ such that for all $b \in \mathbb{T}$,

$$
\sup _{\lambda \in I_{1}, \eta \in(0,1)} \mathbb{E}\left(\left|\operatorname{Im} R_{\lambda+\mathrm{i} \eta}^{+}\left(o_{b}\right)\right|^{-s}\right)<\infty .
$$

Condition (Green-s) implies in particular that the spectrum in $I_{1}$ is purely AC, as long as it stays away from the Dirichlet spectrum, see "Appendix A.2". Here (Green-s) refers to "Green's function" and the moment value $s$. In fact, such inverse bounds on the WT function imply moments bounds on the Green's function; see Corollary 2.1.

Introduce the following assumptions:

(C0) The minimal degree of $\mathbb{T}$ is at least 3.

(C2) For each $k \in \mathfrak{A}$, there is $k^{\prime}$ with $M_{k, k^{\prime}} \geq 1$ such that for any $l \in \mathfrak{A}: M_{k, l} \geq 1$ implies $M_{k^{\prime}, l} \geq 1$.

The second assumption ensures that each vertex $v \in \mathbb{T}$ has at least one child $v^{\prime}$ such that each label found in $\mathscr{N}_{v}^{+}$can also be found in $\mathscr{N}_{v^{\prime}}^{+}$. See [5] for examples of such trees.

Theorem 1.3. Let $\mathbf{T}$ satisfy $(\boldsymbol{C O}),(\boldsymbol{C 1}),(\boldsymbol{C} 2)$ and $(\alpha, L)$ satisfy $(\boldsymbol{P 0}),(\boldsymbol{P 1})$ and $(\boldsymbol{P 2})$, and be without edge potentials. Let $I \subset \Sigma$ be compact with $I \cap \mathscr{D}=\emptyset$. Then for any $s>1$, we may find $\varepsilon_{0}(I, s)$ such that (Green-s) holds on I for any $\varepsilon \leq \varepsilon_{0}$. In particular, $\sigma\left(\mathscr{H}_{\varepsilon}{ }^{\omega}\right)$ has purely absolutely continuous spectrum almost-surely in I.

The "in particular" part is due to Theorem A.1.

In the above theorem, the disorder window $\varepsilon_{0}(I, s)$ depends on the value of the moment $s$. We can actually obtain a disorder window valid uniformly for all $s$, but at the price of assuming some regularity on the $\delta$-potential:

(P3) For any $v \in \mathbb{T}, \ell(v)=j, j \in \mathfrak{A}$, the distribution $v_{j}$ of $\alpha_{v}^{\omega}$ is Hölder continuous: there exist $C_{v}>0$ and $\beta \in(0,1]$ such that for any bounded $I \subset \mathbb{R}$,

$$
\max _{j \in \mathfrak{A}} v_{j}(I) \leq C_{v} \cdot|I|^{\beta} .
$$


This holds e.g. if the $v_{j}$ are absolutely continuous with a bounded density (then $\beta=1$ ).

Theorem 1.4. Suppose in addition to the assumptions of Theorem 1.3 that (P3) is satisfied. Then there exists $\varepsilon_{0}(I)$ such that for any $\varepsilon \leq \varepsilon_{0}$ and any $s \geq 1$, (Green-s) holds on $I$.

We finally mention that assumptions (1.7)-(1.8) in the last two theorems come from our choice to work with the quantity $\frac{R_{z}^{+}\left(o_{b}\right)}{\sqrt{z}}$ in the proof, as it behaves quite nicely with respect to Cayley transforms and transmission from edge to edge. The sign assumption $\alpha_{v} \geq 0$ (and where relevant $W_{v} \geq 0$ ) ensures that $\operatorname{Im} \frac{R_{z}^{+}\left(o_{b}\right)}{\sqrt{z}}>0$ when $\operatorname{Im} z>0$ (see the proof of Lemma 2.3 in "Appendix A.1") This "Herglotz" property is a necessary input for our proof.

\section{Green's Function on Quantum Trees}

The aim of this section is to derive quantum analogs for the well-known recursive formulas of the Green's function on combinatorial trees. These identities will play a key role in the spectral analysis of the quantum tree, and may be of independent interest. In fact, we shall also need them when studying quantum ergodicity in [6]. Some of these identities appeared before in [1].

In all this section, we fix a quantum tree $\mathbf{T}$, and denote by $W_{\max }^{2,2}(\mathbf{T})$ the set of $\psi=\left(\psi_{v}\right)$ such that $\psi_{v} \in W^{2,2}\left(0, L_{v}\right), \sum_{v}\left\|\psi_{v}\right\|_{W^{2,2}}^{2}<\infty$.

If $b \in B(\mathbb{T})$, we write, as in Sect. $1.1, \mathbb{T}_{b}^{+}$for the connected component of $\mathbb{T} \backslash\{e(b)\}$ containing $t_{b}$, and $\mathbb{T}_{b}^{-}$for the component of $\mathbb{T} \backslash\{e(b)\}$ containing $o_{b}$, where $\mathbb{T} \backslash\{e(b)\}$ is the combinatorial tree $\mathbb{T}$ to which we removed the edge corresponding to $b$.

If $x=\left(b, x_{b}\right) \in \mathscr{T}$, we define a quantum tree $\mathbf{T}_{x}^{+}$by $\mathbf{T}_{x}^{+}=\left[x_{b}, t_{b}\right] \cup \mathbf{T}_{b}^{+}$. More precisely, add a vertex $v_{x}$ at $x$, let $V\left(\mathbf{T}_{x}^{+}\right)=V\left(\mathbf{T}_{b}^{+}\right) \cup\left\{v_{x}\right\}, E\left(\mathbf{T}_{x}^{+}\right)=E\left(\mathbf{T}_{b}^{+}\right) \cup\left\{v_{x}, t_{b}\right\}$, $L_{\left\{v_{x}, t_{b}\right\}}=L_{b}-x_{b}, W_{\left(v_{x}, t_{b}\right)}=\left.\left(W_{b}\right)\right|_{\left[L_{b}-x_{b}, L_{b}\right]}, \alpha_{v_{x}}=0$, and the lengths, potentials and coupling constants be the same as those of $\mathbf{T}_{b}^{+}$on the rest of the edges. In a similar fashion, we define $\mathbf{T}_{x}^{-}=\mathbf{T}_{b}^{-} \cup\left[o_{b}, x_{b}\right]$.

Let $u=\left(b, u_{b}\right) \in \mathscr{T}$. By [1, Theorem 2.1], which remains true in our context, if we define $\mathscr{H}_{\mathbf{T}_{u}^{ \pm}}^{\max }$ on $\mathscr{T}_{u}^{ \pm}$to be the Schrödinger operator $-\Delta+W$ with domain $D\left(\mathscr{H}_{\mathbf{T}_{u}^{ \pm}}^{\max }\right)$, the set of $\psi \in W_{\max }^{2,2}\left(\mathbf{T}_{u}^{ \pm}\right)$satisfying $\delta$-conditions on inner vertices of $\mathbf{T}_{u}^{ \pm}$, then for any $z \in \mathbb{C}^{+}:=\mathbb{H}:=\{z \in \mathbb{C}: \operatorname{Im} z>0\}$, there are unique $z$-eigenfunctions $V_{z ; u}^{+} \in$ $D\left(\mathscr{H}_{\mathbf{T}_{u}^{+}}^{\max }\right), U_{z ; u}^{-} \in D\left(\mathscr{H}_{\mathbf{T}_{u}^{-}}^{\max }\right)$ satisfying $U_{z ; u}^{-}(u)=V_{z ; u}^{+}(u)=1$. Complex eigenvalues exist because $\mathscr{H}_{\mathbf{T}_{u}^{ \pm}}^{\max }$ is not self-adjoint, as there are no domain conditions at $u$.

Note that, by uniqueness, if $v, v^{\prime} \in \mathscr{T}$ with $v \in \mathscr{T}_{v^{\prime}}^{-}$, then on $\mathscr{T}_{v}^{-}$, the functions $U_{z ; v}^{-}$ and $U_{z ; v^{\prime}}^{-}$must be proportional. Similarly, $V_{z ; o}^{+}$and $V_{z ; o^{\prime}}^{+}$must be proportional on $\mathscr{T}_{o}^{+}$if $o \in \mathscr{T}_{o^{\prime}}^{+}$. Therefore, the quantity introduced in (2.1) below is independent of the choice of $o$ and $v$.

Lemma 2.1. Let $z \in \mathbb{C}^{+}$. The resolvent $\left(\mathscr{H}_{\mathbf{T}}-z\right)^{-1}$ of $\mathscr{H}_{\mathbf{T}}$ is an integral operator with kernel $G^{z}(x, y)$ defined as follows. Given $x, y \in \mathscr{T}$, fix o, $v$ such that $x, y \in \mathscr{T}_{o}^{+} \cap \mathscr{T}_{v}^{-}$. Then

$$
G^{z}(x, y)= \begin{cases}\frac{U_{z ; v}^{-}(x) V_{z ; o}^{+}(y)}{\mathscr{W}_{v o}^{z}(y)} & \text { if } y \in \mathscr{T}_{x}^{+}, \\ \frac{U_{z ; v}^{-}(y) V_{z ; o}^{+}(x)}{\mathscr{W}_{v, o}^{z}(y)} & \text { if } y \in \mathscr{T}_{x}^{-},\end{cases}
$$


where $\mathscr{W}_{v, o}^{z}(x)$ is the Wronskian

$$
\mathscr{W}_{v, o}^{z}(x)=V_{z ; o}^{+}(x)\left(U_{z ; v}^{-}\right)^{\prime}(x)-\left(V_{z ; o}^{+}\right)^{\prime}(x) U_{z ; v}^{-}(x) .
$$

Versions of this lemma previously appeared in [1, Lemma A.2] and [17, Lemma D.15]. We give the proof in "Appendix A" for completeness.

Since for each $z \in \mathbb{C}^{+}, G^{z}$ satisfies the $\delta$-boundary conditions in each of its arguments, we deduce that, whenever $o_{b}=o_{b^{\prime}}=v$, we have $G^{z}((b, 0), \cdot)=G^{z}\left(\left(b^{\prime}, 0\right), \cdot\right)$ and $G^{z}(\cdot,(b, 0))=G^{z}\left(\cdot,\left(b^{\prime}, 0\right)\right)$. These quantities will therefore be denoted by $G^{z}(v, \cdot)$ and $G^{z}(\cdot, v)$ respectively.

As in [1], we define the Weyl-Titchmarch (WT) functions (which are analogous to Dirichlet-to-Neumann functions) for $x \in \mathscr{T}$ by

$$
R_{z}^{+}(x)=\frac{\left(V_{z ; o}^{+}\right)^{\prime}(x)}{V_{z ; o}^{+}(x)} \text { and } R_{z}^{-}(x)=\frac{-\left(U_{z ; v}^{-}\right)^{\prime}(x)}{U_{z ; v}^{-}(x)}
$$

Note that we take here the coherent point of view, which is why there is a negative sign in the definition of $R_{z}^{-}(x)$.

Given an oriented edge $b=\left(o_{b}, t_{b}\right)$, we define

$$
\zeta^{z}(b)=\frac{G^{z}\left(o_{b}, t_{b}\right)}{G^{z}\left(o_{b}, o_{b}\right)} .
$$

Remark 2.1. If $a<\inf \sigma\left(\mathscr{H}_{\mathbf{T}}\right)$, then $\zeta^{z}(b)$ is well-defined on $\mathbb{C} \backslash(a, \infty)$, i.e. the denominator does not vanish, as follows from (2.1) and the proof of [1, Theorem 2.1(ii)]. The proof also shows that $z \mapsto \zeta^{z}(b)$ is holomorphic on $\mathbb{C} \backslash(a, \infty)$ and real-valued on $(-\infty, a]$.

In the case of combinatorial trees, $\zeta^{z}(b)$ coincides with what was denoted $\zeta_{o_{b}}^{z}\left(t_{b}\right)$ in [3]. In fact, by the multiplicative property of the Green function, we have $\frac{G^{z}\left(o_{b}, t_{b}\right)}{G^{z}\left(o_{b}, o_{b}\right)}=$ $\frac{G^{z}\left(o_{b}, o_{b}\right) \zeta_{o_{b}}^{z}\left(t_{b}\right)}{G^{z}\left(o_{b}, o_{b}\right)}=\zeta_{o_{b}}^{z}\left(t_{b}\right)$.

In the case that $\mathbf{T}$ is the $(q+1)$-regular tree with equilateral edges, with identical coupling constants and potentials, then $\zeta^{z}(b)$ is the quantity $\mu^{-}(z)$ in [12], and is independent of $b$. Moreover, the limit $\mu^{-}(\lambda)=\lim _{\eta \downarrow 0} \mu^{-}(\lambda+i \eta)$ exists in this case, provided that $\lambda$ is not in the Dirichlet spectrum, i.e., that $\sin (\lambda L) \neq 0$.

Finally, for the quantum Cayley graphs of [13], the $\zeta^{z}(b)$ coincide with the multipliers $\mu_{m}(z)$. Hence, there are finitely many distinct $\zeta^{z}(b)$. Moreover, in this setting, $\zeta^{z}(\hat{b})=$ $\zeta^{z}(b)$, and $\zeta^{z}(g b)=\zeta^{z}(b)$, where $g$ is an element of the group acting on the graph.

Given an oriented edge $b$, we will denote by $\mathscr{N}_{b}^{+}$the set of outgoing edges from $b$, i.e. the set of $b^{\prime}$ with $o_{b^{\prime}}=t_{b}$ and $b^{\prime} \neq \hat{b}$.

Lemma 2.2. Let $z \in \mathbb{C}^{+}$. We have the following relations between $\zeta^{z}$ and the $W T$ functions $R_{z}^{ \pm}$:

$$
\begin{aligned}
\zeta^{z}(b)=C_{z}\left(L_{b}\right)+R_{z}^{+}\left(o_{b}\right) S_{z}\left(L_{b}\right), & \zeta^{z}(\hat{b})=S_{z}^{\prime}\left(L_{b}\right)+R_{z}^{-}\left(t_{b}\right) S_{z}\left(L_{b}\right) \\
R_{z}^{+}\left(t_{b}\right)=\frac{S_{z}^{\prime}\left(L_{b}\right)}{S_{z}\left(L_{b}\right)}-\frac{1}{S_{z}\left(L_{b}\right) \zeta^{z}(b)}, & R_{z}^{-}\left(o_{b}\right)=\frac{C_{z}\left(L_{b}\right)}{S_{z}\left(L_{b}\right)}-\frac{1}{S_{z}\left(L_{b}\right) \zeta^{z}(\hat{b})}
\end{aligned}
$$


Moreover,

$$
\begin{aligned}
& \frac{1}{\zeta^{z}(b) S_{z}\left(L_{b}\right)}+\sum_{b^{+} \in \mathscr{N}_{b}^{+}} \frac{\zeta^{z}\left(b^{+}\right)}{S_{z}\left(L_{b^{+}}\right)}=\sum_{b^{+} \in \mathscr{N}_{b}^{+}} \frac{C_{z}\left(L_{b^{+}}\right)}{S_{z}\left(L_{b^{+}}\right)}+\frac{S_{z}^{\prime}\left(L_{b}\right)}{S_{z}\left(L_{b}\right)}+\alpha_{t_{b}}, \\
& \frac{1}{\zeta^{z}(b)}-\zeta^{z}(\hat{b})=\frac{S_{z}\left(L_{b}\right)}{G^{z}\left(t_{b}, t_{b}\right)}, \quad \frac{\zeta^{z}(\hat{b})}{\zeta^{z}(b)}=\frac{G^{z}\left(o_{b}, o_{b}\right)}{G^{z}\left(t_{b}, t_{b}\right)},
\end{aligned}
$$

and

$$
\sum_{b^{+} \in N_{b}^{+}} \frac{C_{z}\left(L_{b^{+}}\right)}{S_{z}\left(L_{b^{+}}\right)}+\frac{S_{z}^{\prime}\left(L_{b}\right)}{S_{z}\left(L_{b}\right)}+\alpha_{t_{b}}=\sum_{t_{b^{\prime}} \sim t_{b}} \frac{\zeta^{z}\left(b^{\prime}\right)}{S_{z}\left(L_{b^{\prime}}\right)}+\frac{1}{G^{z}\left(t_{b}, t_{b}\right)},
$$

where $b^{\prime}=\left(t_{b}, t_{b^{\prime}}\right)$. Given a non-backtracking path $b_{1}, \ldots, b_{k}$ (that is to say, $o_{b_{i+1}}=t_{b_{i}}$ and $t_{b_{i+1}} \neq o_{b_{i}}$ for all $\left.i \in\{1, \ldots, k-1\}\right)$, we have the multiplicative property

$$
G^{z}\left(o_{b_{1}}, t_{b_{k}}\right)=G^{z}\left(o_{b_{1}}, o_{b_{1}}\right) \zeta^{z}\left(b_{1}\right) \cdots \zeta^{z}\left(b_{k}\right)=G^{z}\left(t_{b_{k}}, t_{b_{k}}\right) \zeta^{z}\left(\hat{b}_{1}\right) \cdots \zeta^{z}\left(\hat{b}_{k}\right) .
$$

Finally, for any path $b_{1}, \ldots, b_{k}$, we have

$$
G^{z}\left(o_{b_{1}}, t_{b_{k}}\right)=G^{z}\left(t_{b_{k}}, o_{b_{1}}\right) .
$$

Proof. By (2.1), $\zeta^{z}(b)=\frac{V_{z ; o}^{+}\left(t_{b}\right)}{V_{z ; o}^{+}\left(o_{b}\right)} \cdot \frac{\mathscr{W}_{v, o}^{z}\left(o_{b}\right)}{\mathscr{W}_{v, o}^{z}\left(t_{b}\right)}$. But the Wronskian is constant on $b$, as checked by differentiating it. Moreover, since $V_{z ; o}^{+}\left(x_{b}\right)$ is a $z$-eigenfunction on $b$, we have $V_{z ; o}^{+}\left(t_{b}\right)=C_{z}\left(L_{b}\right) V_{z ; o}^{+}\left(o_{b}\right)+S_{z}\left(L_{b}\right)\left(V_{z ; o}^{+}\right)^{\prime}\left(o_{b}\right)$. Hence, we have as claimed $\zeta^{z}(b)=$ $C_{z}\left(L_{b}\right)+R_{z}^{+}\left(o_{b}\right) S_{z}\left(L_{b}\right)$. Next, $\zeta^{z}(\hat{b})=\frac{G^{z}\left(t_{b}, o_{b}\right)}{G^{z}\left(t_{b}, t_{b}\right)}=\frac{U_{z ; v}^{-}\left(o_{b}\right)}{U_{z ; v}^{-}\left(t_{b}\right)}$ again by constancy of $\mathscr{W}_{v, o}^{z}$ on $b$. Writing (1.6) in the form

$$
\left(\begin{array}{c}
\psi\left(o_{b}, z\right) \\
\psi^{\prime}\left(o_{b}, z\right)
\end{array}\right)=M_{z}(b)^{-1}\left(\begin{array}{c}
\psi\left(t_{b}\right) \\
\psi^{\prime}\left(t_{b}\right)
\end{array}\right)=\left(\begin{array}{cc}
S_{z}^{\prime}\left(L_{b}\right) & -S_{z}\left(L_{b}\right) \\
-C_{z}^{\prime}\left(L_{b}\right) & C_{z}\left(L_{b}\right)
\end{array}\right)\left(\begin{array}{c}
\psi\left(t_{b}\right) \\
\psi^{\prime}\left(t_{b}\right)
\end{array}\right),
$$

we get $U_{z ; v}^{-}\left(o_{b}\right)=S_{z}^{\prime}\left(L_{b}\right) U_{z ; v}^{-}\left(t_{b}\right)-S_{z}\left(L_{b}\right)\left(U_{z ; v}^{-}\right)^{\prime}\left(t_{b}\right)$, so $\zeta^{z}(\hat{b})=S_{z}^{\prime}\left(L_{b}\right)+R_{z}^{-}\left(t_{b}\right) S_{z}$ $\left(L_{b}\right)$ as claimed.

Next, $V_{z ; o}^{+}\left(y_{b}\right)=V_{z ; o}^{+}\left(o_{b}\right) C_{z}\left(y_{b}\right)+\left(V_{z ; o}^{+}\right)^{\prime}\left(o_{b}\right) S_{z}\left(y_{b}\right)$, so

$$
R_{z}^{+}\left(t_{b}\right)=\frac{R_{z}^{+}\left(o_{b}\right) S_{z}^{\prime}\left(L_{b}\right)+C_{z}^{\prime}\left(L_{b}\right)}{R_{z}^{+}\left(o_{b}\right) S_{z}\left(L_{b}\right)+C_{z}\left(L_{b}\right)} .
$$

Using $R_{z}^{+}\left(o_{b}\right)=\frac{\zeta^{z}(b)-C_{z}\left(L_{b}\right)}{S_{z}\left(L_{b}\right)}$, we get

$$
R_{z}^{+}\left(t_{b}\right)=\frac{\zeta^{z}(b) S_{z}^{\prime}\left(L_{b}\right)-C_{z}\left(L_{b}\right) S_{z}^{\prime}\left(L_{b}\right)+C_{z}^{\prime}\left(L_{b}\right) S_{z}\left(L_{b}\right)}{\zeta^{z}(b) S_{z}\left(L_{b}\right)} .
$$

The first part of (2.5) follows by the Wronskian identity $C_{z}(x) S_{z}^{\prime}(x)-C_{z}^{\prime}(x) S_{z}(x)=1$.

For the second part, by (2.11),

$$
R_{z}^{-}\left(o_{b}\right)=\frac{-\left(U_{z ; v}^{-}\right)^{\prime}\left(o_{b}\right)}{U_{z ; v}^{-}\left(o_{b}\right)}=\frac{C_{z}^{\prime}\left(L_{b}\right) U_{z ; v}^{-}\left(t_{b}\right)-C_{z}\left(L_{b}\right)\left(U_{z ; v}^{-}\right)^{\prime}\left(t_{b}\right)}{S_{z}^{\prime}\left(L_{b}\right) U_{z ; v}^{-}\left(t_{b}\right)-S_{z}\left(L_{b}\right)\left(U_{z ; v}^{-}\right)^{\prime}\left(t_{b}\right)}
$$




$$
=\frac{C_{z}\left(L_{b}\right) R_{z}^{-}\left(t_{b}\right)+C_{z}^{\prime}\left(L_{b}\right)}{S_{z}\left(L_{b}\right) R_{z}^{-}\left(t_{b}\right)+S_{z}^{\prime}\left(L_{b}\right)} .
$$

The claim now follows as before using (2.4).

Since $V_{z ; o}^{+}$satisfies the $\delta$-conditions, we have

$$
\sum_{b^{+} \in N_{b}^{+}} R_{z}^{+}\left(o_{b^{+}}\right)=R_{z}^{+}\left(t_{b}\right)+\alpha_{t_{b}}=\frac{S_{z}^{\prime}\left(L_{b}\right)}{S_{z}\left(L_{b}\right)}-\frac{1}{S_{z}\left(L_{b}\right) \zeta^{z}(b)}+\alpha_{t_{b}} .
$$

Recalling (2.4), this proves (2.6).

By (2.1),

$$
\frac{1}{G^{z}\left(t_{b}, t_{b}\right)}=\frac{\mathscr{W}_{v, o}^{z}\left(t_{b}\right)}{U_{z ; v}^{-}\left(t_{b}\right) V_{z ; o}^{+}\left(t_{b}\right)}=\frac{\left(U_{z ; v}^{-}\right)^{\prime}\left(t_{b}\right)}{U_{z ; v}^{-}\left(t_{b}\right)}-\frac{\left(V_{z ; o}^{+}\right)^{\prime}\left(t_{b}\right)}{V_{z ; o}^{+}\left(t_{b}\right)}
$$

so

$$
\frac{1}{G^{z}\left(t_{b}, t_{b}\right)}=-\left(R_{z}^{+}\left(t_{b}\right)+R_{z}^{-}\left(t_{b}\right)\right) .
$$

We have $R_{z}^{+}\left(t_{b}\right)=\frac{S_{z}^{\prime}\left(L_{b}\right)}{S_{z}\left(L_{b}\right)}-\frac{1}{S_{z}\left(L_{b}\right) \zeta^{z}(b)}$ and $R_{z}^{-}\left(t_{b}\right)=\frac{\zeta^{z}(\hat{b})-S_{z}^{\prime}\left(L_{b}\right)}{S_{z}\left(L_{b}\right)}$ by (2.4) and (2.5). Hence,

$$
R_{z}^{+}\left(t_{b}\right)+R_{z}^{-}\left(t_{b}\right)=\frac{-1}{S_{z}\left(L_{b}\right) \zeta^{z}(b)}+\frac{\zeta^{z}(\hat{b})}{S_{z}\left(L_{b}\right)},
$$

proving the first part of (2.7).

For the second part, we showed that $\frac{S_{z}\left(L_{b}\right)}{G^{z}\left(t_{b}, t_{b}\right)}=\frac{1-\zeta^{z}(b) \zeta^{z}(\hat{b})}{\zeta^{z}(b)}$, so replacing $b$ by $\hat{b}$ we deduce that $\frac{S_{z}\left(L_{b}\right)}{G^{z}\left(o_{b}, o_{b}\right)}=\frac{1-\zeta^{z}(\hat{b}) \zeta^{z}(b)}{\zeta^{z}(\hat{b})}$, so $\frac{\zeta^{z}(\hat{b})}{\zeta^{z}(b)}=\frac{G^{z}\left(o_{b}, o_{b}\right)}{G^{z}\left(t_{b}, t_{b}\right)}$.

It follows from (2.13) and (2.14) that

$$
\frac{1}{\zeta^{z}(b) S_{z}\left(L_{b}\right)}=\frac{\zeta^{z}(\hat{b})}{S_{z}\left(L_{b}\right)}+\frac{1}{G^{z}\left(t_{b}, t_{b}\right)} .
$$

Inserting this expression in (2.6), we obtain (2.8).

As previously observed, the Wronskian is constant on each $b$, so $\zeta^{z}(\hat{b})=\frac{U_{z ; v}^{-}\left(o_{b}\right)}{U_{z ; v}^{-}\left(t_{b}\right)}$. Hence,

$$
\begin{aligned}
G^{z}\left(t_{b_{k}}, t_{b_{k}}\right) \zeta^{z}\left(\hat{b}_{1}\right) \cdots \zeta^{z}\left(\hat{b}_{k}\right) & =\frac{U_{z ; v}^{-}\left(v_{k}\right) V_{z ; o}^{+}\left(v_{k}\right)}{\mathscr{W}_{v, o}^{z}\left(v_{k}\right)} \frac{U_{z ; v}^{-}\left(v_{0}\right)}{U_{z ; v}^{-}\left(v_{1}\right)} \cdots \frac{U_{z ; v}^{-}\left(v_{k-1}\right)}{U_{z ; v}^{-}\left(v_{k}\right)} \\
& =\frac{U_{z ; v}^{-}\left(v_{0}\right) V_{z ; o}^{+}\left(v_{k}\right)}{\mathscr{W}_{v, o}^{z}\left(v_{k}\right)}=G^{z}\left(o_{b_{1}}, t_{b_{k}}\right) .
\end{aligned}
$$

By $(2.7), \zeta^{z}\left(b_{1}\right) \cdots \zeta^{z}\left(b_{k}\right)=\zeta^{z}\left(\hat{b}_{1}\right) \cdots \zeta^{z}\left(\hat{b}_{k}\right) \cdot \frac{G^{z}\left(t_{b_{k}}, t_{b_{k}}\right)}{G^{z}\left(o_{b_{1}}, o_{b_{1}}\right)}$, proving the other equality.

Finally, it follows from (2.9) that

$$
G^{z}\left(t_{b_{k}}, o_{b_{1}}\right)=G^{z}\left(t_{b_{k}}, t_{b_{k}}\right) \zeta^{z}\left(\hat{b}_{k}\right) \cdots \zeta^{z}\left(\hat{b}_{1}\right)=G^{z}\left(o_{b_{1}}, t_{b_{k}}\right)
$$


For the following lemma, fix $o, v \in V$ and consider the WT functions (2.2). Assume that $o_{b}, t_{b} \in V\left(\mathbf{T}_{o}^{+}\right) \cap V\left(\mathbf{T}_{v}^{-}\right)$, that is to say, that $b \in B\left(\mathbf{T}_{o}^{+}\right)$and $\hat{b} \in B\left(\mathbf{T}_{v}^{-}\right)$. Let $\mathbf{T}_{o_{b}}^{+} \subseteq \mathbf{T}_{o}^{+}$ and $\mathbf{T}_{t_{b}}^{-} \subseteq \mathbf{T}_{v}^{-}$be the subtrees starting at $o_{b}$ and $t_{b}$, respectively. Let $G_{\mathbf{T}_{o_{b}}^{+}}(x, y)$ be the Green kernel of the $\delta$-problem on $\mathbf{T}_{o_{b}}^{+}$. This means the usual $\delta$-conditions at $v \in V\left(\mathbf{T}_{o_{b}}^{+}\right)$, with $\alpha_{o_{b}}=0$. Similarly, $G_{\mathbf{T}_{t_{b}}^{-}}(x, y)$ is the Green kernel of the $\delta$-problem on $\mathbf{T}_{t_{b}}^{-}$.

We will need the notion of Herglotz functions [14] throughout the paper. A Herglotz function (a.k.a. Nevanlinna function or Pick function) is an analytic function from $\mathbb{C}^{+}$ to $\mathbb{C}^{+}$. Herglotz functions form a positive cone: if $f_{1}, f_{2}$ are Herglotz and $a_{1}, a_{2}$ are positive constants, then $a_{1} f_{1}+a_{2} f_{2}$ is Herglotz. Composition of two Herglotz functions is again a Herglotz function. The functions $z \mapsto \sqrt{z}$ and $z \mapsto-1 / z$ for example are Herglotz. form

Every Herglotz function $f$ has a canonical representation [14, Theorem II.I] of the

$$
f(z)=A z+B+\int_{\mathbb{R}}\left(\frac{1}{t-z}-\frac{t}{1+t^{2}}\right) \mathrm{d} \mathfrak{m}(t),
$$

where $A$ and $B$ are constants and $\mathfrak{m}$ is a Borel measure satisfying $\int_{\mathbb{R}}\left(1+t^{2}\right)^{-1} \mathrm{dm}<\infty$.

Lemma 2.3. Let $b \in \mathbf{T}$ and $z \in \mathbb{C}^{+}$. Let $o, v \in V$ be such that $b \in \mathbf{T}_{o}^{+}$and $\hat{b} \in \mathbf{T}_{v}^{-}$. Then we may express

$$
R_{z}^{+}\left(o_{b}\right)=\frac{-1}{G_{\mathbf{T}_{b}^{+}}^{z}\left(o_{b}, o_{b}\right)} \quad \text { and } \quad R_{z}^{-}\left(t_{b}\right)=\frac{-1}{G_{\mathbf{T}_{t_{b}}^{-}}^{z}\left(t_{b}, t_{b}\right)},
$$

where $G_{\mathbf{T}_{v}^{ \pm}}^{z}(v, v)$ are defined with the Neumann condition at $v$.

The functions $F(z)=R_{z}^{+}\left(o_{b}\right), R_{z}^{-}\left(t_{b}\right)$ and $G^{z}(v, v)$ are Herglotz functions. If all $W_{v} \geq 0$ and $\alpha_{v} \geq 0$, then $\widetilde{F}(z)=\frac{R_{z}^{+}\left(o_{b}\right)}{\sqrt{z}}, \frac{R_{z}^{-}\left(t_{b}\right)}{\sqrt{z}}$ are also Herglotz.

Moreover, we have the following "current" relations:

$$
\sum_{b^{+} \in \mathscr{N}_{b}^{+}} \operatorname{Im} R_{z}^{+}\left(o_{b^{+}}\right) \leq \frac{\operatorname{Im} R_{z}^{+}\left(o_{b}\right)}{\left|\zeta^{z}(b)\right|^{2}} \quad \text { and } \quad \sum_{b^{-} \in \mathscr{N}_{b}^{-}} \operatorname{Im} R_{z}^{-}\left(t_{b^{-}}\right) \leq \frac{\operatorname{Im} R_{z}^{-}\left(t_{b}\right)}{\left|\zeta^{z}(\hat{b})\right|^{2}}
$$

Equality holds in both cases if $\operatorname{Im} z=0$, whenever defined.

Most statements of this lemma appear in [1]. We give the proof in "Appendix A" for completeness. We also deduce that $-\frac{S_{z}^{\prime}\left(L_{b}\right)}{S_{z}\left(L_{b}\right)}$ and $S_{z}\left(L_{b}\right) \zeta^{z}(b) \in \mathbb{C}^{+}$, see Remark A.2.

The following corollary says that the inverse moments of the imaginary part of the WT functions essentially control all relevant spectral quantities on the tree:

Corollary 2.1. Let $I \subset \mathbb{R}$ be compact, $I \cap \mathscr{D}=\emptyset$, and $z \in \mathbb{C}^{+}$. Fix $c_{1}, c_{2}, c_{3}>0$ such that for all $z \in I+\mathrm{i}[0,1], L_{b} \in\left[L_{\min }, L_{\max }\right]$,

$$
c_{1} \leq\left|S_{z}\left(L_{b}\right)\right| \leq c_{2} \quad \text { and } \quad\left|C_{z}\left(L_{b}\right)\right| \leq c_{3} .
$$

Then for any $p \geq 1$, and $b \in \mathbb{T}$,

$$
\left|G^{z}\left(o_{b}, o_{b}\right)\right|^{p} \leq\left|\operatorname{Im} R_{z}^{+}\left(o_{b}\right)\right|^{-p}, \quad\left|\zeta^{z}(b)\right|^{p} \leq c_{1}^{-p} \sum_{b^{+} \in \mathscr{N}_{b}^{+}}\left|\operatorname{Im} R_{z}^{+}\left(o_{b^{+}}\right)\right|^{-p}
$$


and

$$
\left|R_{z}^{+}\left(o_{b}\right)\right|^{p} \leq c_{1}^{-p} 2^{p-1}\left(c_{1}^{-p} \sum_{b^{+} \in \mathscr{N}_{b}^{+}}\left|\operatorname{Im} R_{z}^{+}\left(o_{b^{+}}\right)\right|^{-p}+c_{3}^{p}\right) .
$$

Also note that $R_{z}^{-}\left(t_{b}\right)=R_{z}^{+}\left(o_{\widehat{b}}\right)+\frac{C_{z}\left(L_{b}\right)-S_{z}^{\prime}\left(L_{b}\right)}{S_{z}\left(L_{b}\right)}$ using (2.4), $\mathrm{so}^{3}$ up to choosing $c_{4}>0$ with $\left|S_{z}^{\prime}\left(L_{b}\right)\right| \leq c_{4}$, a control over all $R_{z}^{+}\left(o_{b}\right), b \in \mathbb{T}$, implies a control over all $R_{z}^{-}\left(t_{b}\right)$.

Proof. We have by (2.13), $\left|G^{z}\left(o_{b}, o_{b}\right)\right|^{p}=\left|R_{z}^{+}\left(o_{b}\right)+R_{z}^{-}\left(o_{b}\right)\right|^{-p} \leq\left|\operatorname{Im} R_{z}^{+}\left(o_{b}\right)\right|^{-p}$. By (2.12),

$$
\begin{aligned}
\left|\zeta^{z}(b)\right|^{p} \leq c_{1}^{-p}\left|S_{z}\left(L_{b}\right) \zeta^{z}(b)\right|^{p} & \leq c_{1}^{-p}\left|\alpha_{t_{b}}+\frac{S_{z}^{\prime}\left(L_{b}\right)}{S_{z}\left(L_{b}\right)}-\sum_{b^{+} \in \mathscr{N}_{b}^{+}} R_{z}^{+}\left(o_{b^{+}}\right)\right|^{-p} \\
& \leq c_{1}^{-p}\left|\operatorname{Im}\left(\alpha_{t_{b}}+\frac{S_{z}^{\prime}\left(L_{b}\right)}{S_{z}\left(L_{b}\right)}-\sum_{b^{+} \in \mathscr{N}_{b}^{+}} R_{z}^{+}\left(o_{b^{+}}\right)\right)\right|^{-p} \\
& \leq c_{1}^{-p}\left(\sum_{b^{+} \in \mathscr{N}_{b}^{+}} \operatorname{Im} R_{z}^{+}\left(o_{b^{+}}\right)\right)^{-p} \leq c_{1}^{-p}\left|\operatorname{Im} R_{z}^{+}\left(o_{b^{+}}\right)\right|^{-p},
\end{aligned}
$$

where we used that $-\frac{S_{z}^{\prime}\left(L_{b}\right)}{S_{z}\left(L_{b}\right)}$ and $R_{z}^{+}\left(o_{e}\right)$ are Herglotz in the last line, with $b^{+} \in \mathscr{N}_{b}^{+}$ arbitrary. Hence,

$$
\begin{aligned}
\left|R_{z}^{+}\left(o_{b}\right)\right|^{p} & =\left|\frac{\zeta^{z}(b)-C_{z}\left(L_{b}\right)}{S_{z}\left(L_{b}\right)}\right|^{p} \leq c_{1}^{-p} 2^{p-1}\left(\left|\zeta^{z}(b)\right|^{p}+c_{3}^{p}\right) \\
& \leq c_{1}^{-p} 2^{p-1}\left(c_{1}^{-p}\left|\operatorname{Im} R_{z}^{+}\left(o_{b^{+}}\right)\right|^{-p}+c_{3}^{p}\right) .
\end{aligned}
$$

\section{AC Spectrum for the Unperturbed Tree}

The aim of this section is to prove Theorems 1.1 and 1.2.

Let $\mathbf{T}$ be a quantum tree of finite cone type, with the structure described in Sect. 1.2. Given $\left(v_{-}, v\right) \in B\left(\mathbb{T}_{b_{o}}^{+}\right)$, we denote

$$
\zeta^{z}(v)=\frac{G^{z}\left(v_{-}, v\right)}{G^{z}\left(v_{-}, v_{-}\right)}
$$

This notation is simply analogous to the one introduced in Sect. 1.1.2, and does not mean that $\zeta^{z}$ is a function of the terminus alone. It simply means that each discrete edge in $\mathbb{T}_{b_{o}}^{+}$can be specified by indicating the terminus alone. We also let $\zeta^{z}\left(t_{b_{o}}\right)=\zeta^{z}\left(b_{o}\right)$.

Denote $\zeta_{j}^{z}=\zeta^{z}(v)$ if $\ell(v)=j$. Then (2.6) says that for each $j \in \mathfrak{A}^{+}$,

$$
\frac{1}{\zeta_{j}^{z} S_{z}\left(L_{j}\right)}+\sum_{k=1}^{m} M_{j, k} \frac{\zeta_{k}^{z}}{S_{z}\left(L_{k}\right)}=\sum_{k=1}^{m} M_{j, k} \frac{C_{z}\left(L_{k}\right)}{S_{z}\left(L_{k}\right)}+\frac{S_{z}^{\prime}\left(L_{j}\right)}{S_{z}\left(L_{j}\right)}+\alpha_{j} .
$$

\footnotetext{
3 If $W_{b}$ is symmetric, i.e. $W_{b}\left(L_{b}-x_{b}\right)=W_{b}\left(x_{b}\right)$, then $S_{z}^{\prime}\left(L_{b}\right)=C_{z}\left(L_{b}\right)$, so $R_{z}^{-}\left(t_{b}\right)=R_{z}^{+}\left(o_{\widehat{b}}\right)$.
} 
The matrix elements $M_{j, k}$ were defined in Sect. 1.2(b). The system (3.1) is reminiscent of the finite system of equations that appears in the combinatorial case $[5,19]$ for $\zeta_{j}^{z}=$ $\zeta_{v_{-}}^{z}(v)$. In order to put it in a nicer form, we denote $h_{j}=S_{z}\left(L_{j}\right) \zeta_{j}^{z}$. Then we get the following system of polynomial equations:

$$
\sum_{k=1}^{m} \frac{M_{j, k}}{S_{z}^{2}\left(L_{k}\right)} h_{k} h_{j}-F_{j}(z) h_{j}+1=0, \quad j=1, \ldots, m
$$

where $F_{j}(z)=\alpha_{j}+\sum_{k=1}^{m} M_{j, k} \frac{C_{z}\left(L_{k}\right)}{S_{z}\left(L_{k}\right)}+\frac{S_{z}^{\prime}\left(L_{j}\right)}{S_{z}\left(L_{j}\right)}$.

An analogous system of equations involving the matrix $N=\left(N_{i, j}\right)$ arises when considering cones in $\mathbb{T}_{b_{o}}^{-}$. We restrict ourselves to the above system; the other one is analyzed similarly.

We mention that a similar system of equations in a more special framework appeared recently in [13, Eq. (4.8)]. In this case, one has $M_{j, j}=1$ for each $j$ and $M_{j, k}=2$ for $k \neq j$.

Our aim in the following is to control the values of $\zeta_{j}^{\lambda+i \eta}$ as $\eta \downarrow 0$. For the models $[12,13]$, the $\zeta_{j}^{z}$ are uniformly bounded. The following simple criterion gives a sufficient condition for this to happen. Note the condition $M_{j, j}>0$ below implies that each vertex of label $j$ has at least one offspring of its own type. Later we will relax that restriction.

Lemma 3.1. Suppose $M_{j, j}>0$ for some $j$. Then $\left|\zeta_{j}^{z}\right|<1$ for any $z \in \mathbb{C} \backslash \mathbb{R}$. In fact, $\left|\zeta_{j}^{z}\right|^{2}<\frac{1}{M_{j, j}}$

This lemma parallels the combinatorial case [19, Lemma 3], see [13, Lemma 3.9] for a special case.

Proof. Let $z \in \mathbb{C}^{+}$and $b$ with $\ell\left(t_{b}\right)=j$. Then (2.17) becomes $\sum_{k=1}^{m} M_{j, k} \operatorname{Im} R_{z}^{+}(k) \leq$ $\frac{\operatorname{Im} R_{z}^{+}(j)}{\left|\zeta_{j}^{z}\right|^{2}}$, where $R_{z}^{+}(k):=R_{z}^{+}\left(o_{e}\right)$ if $\ell\left(t_{e}\right)=k$. The inequality is actually strict if $\operatorname{Im} z>$ 0 , as seen from the proof of (2.17). Thus, $\left|\zeta_{j}^{z}\right|^{2}<\frac{\operatorname{Im} R_{z}^{+}(j)}{M_{j, j} \operatorname{Im} R_{z}^{+}(j)}=\frac{1}{M_{j, j}}$.

The case $\operatorname{Im} z<0$ can be adapted without difficulty, in this case $\operatorname{Im} R_{z}^{+}\left(o_{e}\right)$ should be replaced by $\left|\operatorname{Im} R_{z}^{+}\left(o_{e}\right)\right|$ in (2.17).

The lemma implies in particular that $\left|\zeta_{j}^{\lambda+\mathrm{i} 0}\right| \leq \frac{1}{M_{j, j}}$ for any $\lambda \in \mathbb{R}$.

There are many models of interest for which the condition of Lemma 3.1 is not satistfied, so we next consider the general case. Now the limit $\zeta_{j}^{\lambda+\mathrm{i} 0}$ may no longer exist, but we aim to show this problem can only occur on a discrete subset of $\mathbb{R}$.

Proposition 3.1. There is a discrete set $\mathfrak{D} \subset \mathbb{R}$ such that, for all $j=1, \ldots, m$, the solutions $h_{j}(\lambda+\mathrm{i} \eta)=S_{\lambda+\mathrm{i} \eta}\left(L_{j}\right) \zeta_{j}^{\lambda+\mathrm{i} \eta}$ of (3.2) have a finite limit as $\eta \downarrow 0$ for all $\lambda \in \mathbb{R} \backslash \mathfrak{D}$. The map $\lambda \mapsto S_{\lambda}\left(L_{j}\right) \zeta_{j}^{\lambda+\mathrm{i} 0}$ is continuous on $\mathbb{R} \backslash \mathfrak{D}$, and there is a discrete set $\mathfrak{D}^{\prime}$ such that it is analytic on $\mathbb{R} \backslash\left(\mathfrak{D} \cup \mathfrak{D}^{\prime}\right)$.

Proof. We follow the strategy in [5, Sect. 4]. The aim is essentially to decouple the system (3.2) and show that each $h_{j}$ satisfies an algebraic equation $Q_{j}\left(h_{j}\right)=0$. For this, we will use an algebraic tool from [24].

Let $\lambda_{0} \in \mathbb{R}$, and let $P_{j}\left(h_{1}, \ldots, h_{m}\right)=\sum_{k=1}^{m} \frac{M_{j, k}}{S_{z}^{2}\left(L_{k}\right)} h_{k} h_{j}-F_{j}(z) h_{j}+1$. Clearly, $P_{j} \in K\left[h_{1}, \ldots, h_{m}\right]$, where $K=\mathscr{K}_{\lambda_{0}}$ is the field of functions $f(z)$ possessing a 
convergent Laurent series $f(z)=\sum_{j=-n_{0}}^{\infty} a_{j}\left(\lambda_{0}\right)\left(z-\lambda_{0}\right)^{j}$ in some neighbourhood $N_{\lambda_{0}} \subset \mathbb{C}$ of $\lambda_{0}$.

Let $K^{\prime}=\mathscr{J}_{\lambda_{0}}$ be the field of functions $f$ which are meromorphic on $N_{\lambda_{0}} \cap \mathbb{C}^{+}$for some neighbourhood $N_{\lambda_{0}}$ of $\lambda_{0}$. Then $K^{\prime}$ is an extension of $K$, and we know that $S_{z}\left(L_{j}\right) \zeta_{j}^{z}$ belongs to $K^{\prime}$ (see Remark 2.1) and satisfy $P_{j}\left(S_{z} \zeta_{1}^{z}, \ldots, S_{z} \zeta_{m}^{z}\right)=0$. Calculating the Jacobian $\left(\frac{\partial P_{j}}{\partial h_{k}}(h)\right)$, we find

$$
\frac{\partial P_{j}}{\partial h_{k}}=\left\{\begin{array}{lr}
\frac{M_{j, k}}{S_{z}^{2}\left(L_{j}\right)} h_{j}, & k \neq j, \\
\sum_{\ell=1}^{m} \frac{M_{\ell, j}}{S_{z}^{2}\left(L_{\ell}\right)} h_{\ell}+\frac{M_{j, j}}{S_{z}^{2}\left(L_{j}\right)} h_{j}-F_{j}(z), & k=j .
\end{array}\right.
$$

Let

$$
J^{z}=\left.\operatorname{det}\left(\frac{\partial P_{j}}{\partial h_{k}}\right)\right|_{h=S_{z}\left(L_{k}\right) \zeta_{k}^{z}} \cdot
$$

We will show that $z \mapsto J^{z}$ is not the zero element of $K^{\prime}$. For this, we first study the asymptotics of $J^{z}$ as $z \rightarrow-\infty$.

Take $z=-r^{2}$ with $r>0$ large. We remark that

$$
\lim _{r \rightarrow \infty} \frac{C_{-r^{2}}\left(L_{k}\right)}{r S_{-r^{2}}\left(L_{k}\right)}=1 \quad \text { and } \quad \lim _{r \rightarrow \infty} \frac{S_{-r^{2}}^{\prime}\left(L_{k}\right)}{r S_{-r^{2}}\left(L_{k}\right)}=1 .
$$

This follows from classical estimates [26, Chapter 1]. In fact,

$$
S_{-r^{2}}(L) \approx \frac{\sin \mathrm{i} r L}{\mathrm{i} r}=\frac{\sinh r L}{r}, \quad C_{-r^{2}}(L) \approx \cos \mathrm{i} r L=\cosh r L \approx S_{-r^{2}}^{\prime}(L) .
$$

More precisely, we write

$$
\frac{C_{-r^{2}}(L)}{r S_{-r^{2}}(L)}=\frac{\cosh r L+R(r, L)}{\sinh r L+r R^{\prime}(r, L)}
$$

where $R(r, L)=C_{-r^{2}}(L)-\cosh r L$ and $R^{\prime}(r, L)=S_{-r^{2}}(L)-\frac{\sinh r L}{r}$. By [26, p. 13], $\frac{r R^{\prime}(r, L)}{\sinh r L} \rightarrow 0$ and $\frac{R(r, L)}{\sinh r L} \rightarrow 0$ as $r \rightarrow \infty$. Since $\frac{\cosh r L}{\sinh r L} \rightarrow 1$, (3.3) follows. Hence,

$$
F_{j}\left(-r^{2}\right) \sim \alpha_{j}+r+r \sum_{k} M_{j, k} \sim C_{j} r \quad \text { as } r \rightarrow \infty .
$$

On the other hand, since $h_{j}$ is Herglotz (see Remark A.2), it has a representation of the form (2.15). If $t_{0}=\inf \sigma\left(\mathscr{H}_{\mathbf{T}}\right)$, we also know from Remark 2.1 that $h_{j}(\lambda)$ is well-defined and real-valued for $\lambda<t_{0}$. By [32, Theorem 3.23], the measure $\mathfrak{m}$ is thus supported on $\left[t_{0}, \infty\right)$. Hence, for large $r$ (say $\left.r^{2}>-t_{0}+1\right)$,

$$
h_{j}\left(-r^{2}\right)=-A r^{2}+B+\int_{t_{0}}^{\infty} \frac{1-r^{2} t}{t+r^{2}} \frac{\mathrm{dm}(t)}{1+t^{2}},
$$

where we used that $h_{j}\left(-r^{2}\right)=\lim _{\eta \downarrow} h_{j}\left(-r^{2}+\mathrm{i} \eta\right)$ and dominated convergence (recall that $\frac{\mathrm{d} \mathfrak{m}(t)}{1+t^{2}}$ is a finite measure). Thus,

$$
\frac{h_{j}\left(-r^{2}\right)}{-r^{2}}=A-\frac{B}{r^{2}}+\int_{t_{0}}^{\infty} \frac{t-\frac{1}{r^{2}}}{t+r^{2}} \frac{\mathrm{dm}(t)}{1+t^{2}} .
$$


Using dominated convergence again, we see that $h_{j}\left(-r^{2}\right) /\left(-r^{2}\right) \rightarrow A$ as $r \rightarrow \infty$. This implies that

$$
\frac{h_{j}\left(-r^{2}\right)}{S_{-r^{2}}^{2}\left(L_{k}\right)}=O\left(r^{4} \mathrm{e}^{-2 r L_{k}}\right)=O\left(\mathrm{e}^{-r L_{k}}\right) .
$$

Therefore, recalling that the $C_{j}$ were defined in (3.4), we find that as $r \rightarrow \infty$,

$$
J^{-r^{2}}=\operatorname{det}\left(\begin{array}{cccc}
-C_{1} r+O\left(\mathrm{e}^{-r L_{1}}\right) & O\left(\mathrm{e}^{-r L_{2}}\right) & \cdots & O\left(\mathrm{e}^{-r L_{m}}\right) \\
O\left(\mathrm{e}^{-r L_{1}}\right) & -C_{2} r+O\left(\mathrm{e}^{-r L_{2}}\right) & \cdots & O\left(\mathrm{e}^{-r L_{m}}\right) \\
\vdots & \vdots & \ddots & \vdots \\
O\left(\mathrm{e}^{-r L_{1}}\right) & O\left(\mathrm{e}^{-r L_{2}}\right) & \cdots & -C_{m} r+O\left(\mathrm{e}^{-r L_{m}}\right)
\end{array}\right)
$$

Hence, $J^{-r^{2}} \sim(-1)^{m} C_{1} \cdots C_{m} r^{m}=C r^{m} \neq 0$ for $r$ large enough. Since $z \mapsto J^{z}$ is holomorphic on $\mathbb{C} \backslash\left[a_{0}, \infty\right)$, it follows that it cannot vanish identically on any neighbourhood $N_{\lambda_{0}} \cap \mathbb{C}^{+}$. Hence, $J^{z}$ is not the zero element of $K^{\prime}$.

It follows by [24, Proposition VIII.5.3] that each $S_{z} \zeta_{j}^{z}$ is algebraic over $K$. By the Newton-Puiseux theorem (see e.g. [29, Theorem 3.5.2]), each $h_{j}$ thus has an expansion of the form

$$
h_{j}(z)=\sum_{n \geq m} a_{n}\left(z-\lambda_{0}\right)^{n / d}
$$

in some neighbourhood $N_{\lambda_{0}}$ of $\lambda_{0}$. Here $m \in \mathbb{Z}, d \in \mathbb{N}$, and the entire series $\sum_{n \geq 0} a_{n} z^{n}$ has a positive radius of convergence. In particular, $z \mapsto S_{z} \zeta_{j}^{z}$ is analytic near any $\lambda \in N_{\lambda_{0}} \backslash\left\{\lambda_{0}\right\}$. The set $\mathfrak{D}$ corresponds to those $\lambda_{0}$ for which $m<0$ in the NewtonPuiseux expansion at $\lambda_{0}$, and the set $\mathfrak{D}^{\prime}$ corresponds to those $\lambda_{0}$ for which $d>1$.

Since, for any $\lambda_{0}$, the map $z \mapsto h_{j}(z)$ is analytic in $N_{\lambda_{0}} \backslash\left\{\lambda_{0}\right\}$, we see that, for any $\lambda_{0}$, we have $\left(\mathfrak{D} \cup \mathfrak{D}^{\prime}\right) \cap\left(N_{\lambda_{0}} \backslash\left\{\lambda_{0}\right\}\right)=\emptyset$. Therefore, the sets $\mathfrak{D}$ and $\mathfrak{D}^{\prime}$ are discrete.

Our next aim is to show that all WT functions have a positive imaginary part on most of the spectrum.

Let $\sigma_{D}$ be the union of the Dirichlet spectra:

$$
\sigma_{D}=\bigcup_{j=1}^{m}\left\{\lambda \in \mathbb{R}: S_{\lambda}\left(L_{j}\right)=0\right\} .
$$

We would like to index the WT functions by vertices, but the notation $R_{z}^{+}(v)$ is a bit ambiguous since $R_{z}^{+}\left(t_{b}\right) \neq R_{z}^{+}\left(o_{b^{+}}\right)$even if $t_{b}=o_{b^{+}}=v$. So we take the convention that

$$
R_{z}^{ \pm}(v):= \begin{cases}R_{z}^{ \pm}\left(o_{b}\right) & \text { if } b=\left(v_{-}, v\right) \in \mathbb{T}_{b_{o}}^{+} \\ R_{z}^{ \pm}\left(t_{b}\right) & \text { if } b=\left(v, v_{+}\right) \in \mathbb{T}_{b_{o}}^{-}\end{cases}
$$

Here $\left(t_{b_{o}}\right)_{-}=o_{b_{o}}$ and $\left(o_{b_{o}}\right)_{+}=t_{b_{o}}$. This keeps with the convention of Sect. 1.1.2 of indexing functions $\psi(b)$ by their terminus ${ }^{4}$ on $\mathbb{T}_{b_{o}}^{+}$and their origin on $\mathbb{T}_{b_{o}}^{-}$.

\footnotetext{
4 The notation is probably a bit awkward since for $v \in \mathbb{T}_{b_{o}}^{+}, v$ is the terminus of $b$ yet $R_{z}^{ \pm}(v):=R_{z}^{ \pm}\left(o_{b}\right)$. We stress however that $R_{z}^{ \pm}$is not a function of the vertex $o_{b}$ alone but depends on the whole directed edge $b$, so it should really be read as a function $\psi(b)$, which we index by the terminus.
} 
As there are finitely many types of $\zeta^{z}(b)$ for $b=\left(v_{-}, v\right) \in B\left(\mathbb{T}_{b_{o}}^{+}\right)$, we see by (2.4) there are finitely many types of $R_{z}^{+}\left(o_{b}\right)$ (this may not be true for $R_{z}^{-}\left(o_{b}\right)$ for such $b$ ). We denote $R_{z}^{+}(j):=R_{z}^{+}\left(o_{b}\right)$ if $b=\left(v_{-}, v\right) \in \mathbb{T}_{b_{o}}^{+}$and $\ell(v)=j \in \mathfrak{A}^{+}$. Similarly, we denote $R_{z}^{-}(k)=R_{z}^{-}\left(t_{b}\right)$ if $b=\left(v, v_{+}\right) \in \mathbb{T}_{b_{o}}^{-}$and $\ell(v)=k \in \mathfrak{A}^{-}$.

By some abuse of notation, we assume the discrete sets $\mathfrak{D}, \mathfrak{D}^{\prime}$ of Proposition 3.1 are the same for the system analogous to (3.2) which involves the matrix $\left(N_{i, j}\right)$.

Remark 3.1. Denote $R_{\lambda}^{ \pm}:=R_{\lambda+\mathrm{i} 0}^{ \pm}$. Then the limits $R_{\lambda}^{+}(j)$ exist for $\lambda \in \mathbb{R} \backslash\left(\mathfrak{D} \cup \sigma_{D}\right)$ and $j \in \mathfrak{A}^{+}$. This follows from Proposition 3.1 and (2.4), which implies that $R_{\lambda}^{+}(j)=$ $\frac{\zeta_{j}^{\lambda}-C_{\lambda}\left(L_{j}\right)}{S_{\lambda}\left(L_{j}\right)}$. Similarly, the limits $R_{\lambda}^{-}(k)$ exist for $k \in \mathfrak{A}^{-}$.

It follows that $R_{\lambda}^{ \pm}(v)$ exist for $v \in\left\{o_{b_{o}}, t_{b_{o}}\right\}$ and $\lambda \notin \mathfrak{D} \cup \sigma_{D}$. In fact, $\zeta^{\lambda}\left(b_{o}\right)=\frac{h_{j_{o}}}{S_{\lambda}\left(L_{o}\right)}$ for some $j_{o} \in \mathfrak{A}^{+}$, which exists by Proposition 3.1, so $R_{\lambda}^{+}\left(o_{b_{o}}\right)$ exists by (2.4). Similarly the result for the $\left(N_{i j}\right)$ system implies the existence of $\zeta^{\lambda}\left(\hat{b}_{o}\right)$ and $R_{\lambda}^{-}\left(t_{b_{o}}\right)$. Finally if $t_{b_{o}}$ has type $j_{o} \in \mathfrak{A}^{+}$, then $R_{\lambda}^{+}\left(t_{b_{o}}\right)=\sum_{k=1}^{m} M_{j_{o}, k} R_{\lambda}^{+}(k)-\alpha_{t_{b_{o}}}$ by (2.12), which exists by the previous paragraph. Similarly $R_{\lambda}^{-}\left(o_{b_{o}}\right)=\sum_{k=1}^{n} N_{j_{o}^{\prime}, k} R_{\lambda}^{-}(k)+\alpha_{o_{b_{o}}}$ exists.

Proposition 3.1 tells us moreover that $R_{\lambda+\mathrm{i} 0}^{ \pm}(v)$ are analytic on $\mathbb{R} \backslash\left(\mathfrak{D} \cup \mathfrak{D}^{\prime} \cup \sigma_{D}\right)$. In particular, their zeroes do not accumulate. Hence, there is a discrete set $\mathfrak{D}^{\prime \prime}$ such that $R_{\lambda}^{+}(v)+R_{\lambda}^{-}(v) \neq 0$ for $\lambda \in \mathbb{R} \backslash\left(\mathfrak{D} \cup \mathfrak{D}^{\prime} \cup \mathfrak{D}^{\prime \prime} \cup \sigma_{D}\right)$ and $v \in\left\{o_{b_{o}}, t_{b_{o}}\right\}$. We therefore define

$$
\mathfrak{D}_{0}:=\mathfrak{D} \cup \mathfrak{D}^{\prime} \cup \mathfrak{D}^{\prime \prime} \cup \sigma_{D}
$$

We may actually generalize the result of Remark 3.1 as follows.

Lemma 3.2.(a) If $\lambda \notin \mathfrak{D} \cup \sigma_{D}$, then $R_{\lambda}^{ \pm}\left(o_{b}\right)$ exists for all $b=\left(v_{-}, v\right) \in \mathbb{T}_{b_{o}}^{+}$and $R_{\lambda}^{ \pm}\left(t_{b}\right)$ exists for all $b=\left(w, w_{+}\right) \in \mathbb{T}_{b_{o}}^{-}$.

(b) If moreover $\lambda \notin \mathfrak{D}_{0}$, then $G^{\lambda}(v, v)$ exists for any $v \in \mathbb{T}$, and $G^{\lambda}(v, v) \neq 0$.

Proof. By symmetry it suffices to prove (a) for $\mathbb{T}_{b_{o}}^{+}$. Consider $b=\left(v_{-}, v\right) \in \mathbb{T}_{b_{o}}^{+}$. We already know that $R_{\lambda}^{+}\left(o_{b}\right)=R_{\lambda}^{+}(j)$ exists from Remark 3.1. Next, we show by induction that $\frac{1}{\zeta^{\lambda}(\widehat{b}) S_{\lambda}\left(L_{b}\right)}$ is finite. Note that we already know $\frac{1}{\zeta^{\lambda}\left(\widehat{b}_{o}\right) S_{\lambda}\left(L_{o}\right)}$ is finite by (2.5). So consider any oriented edge $b=\left(t_{b_{o}}, v\right)$. Applying (2.6) to $\widehat{b}$ instead of $b$, we may express $\frac{1}{\zeta^{z}(\widehat{b}) S_{z}\left(L_{b}\right)}$ in terms of some $C_{z}, S_{z}$ functions, plus $\zeta^{z}\left(b^{\prime}\right)$ for $b^{\prime} \in \mathscr{N}_{\widehat{b}}^{+}$. One of them is $\zeta^{z}\left(\widehat{b}_{o}\right)$, whose limit on the real axis exists from Remark 3.1. The rest are precisely those with $b^{\prime} \in \mathscr{N}_{b_{o}}^{+} \backslash\{b\}$, which also exist by Proposition 3.1. Thus, $\frac{1}{\zeta^{\lambda}(\vec{b}) S_{\lambda}\left(L_{b}\right)}$ exists for any $b=\left(t_{b_{o}}, v\right)$. By induction we get existence for any $\left(v_{-}, v\right) \in \mathbb{T}_{b_{o}}^{+}$. It follows from (2.5) that $R_{\lambda}^{-}\left(o_{b}\right)$ exists for all such $b=\left(v_{-}, v\right) \in \mathbb{T}_{b_{o}}^{+}$.

(b) By Remark 3.1, $R_{\lambda}^{+}(v)+R_{\lambda}^{-}(v) \neq 0$ for $v=o_{b_{o}}, t_{b_{o}}$. Using (2.13), this implies $G^{\lambda}(v, v)$ exists. We now observe that

$$
G^{z}\left(v_{+}, v_{+}\right)=S_{z}\left(L_{v}\right) \zeta^{z}\left(v, v_{+}\right)+\zeta^{z}\left(v, v_{+}\right)^{2} G^{z}(v, v) .
$$

In fact, by (2.7),

$$
G^{z}\left(v_{+}, v_{+}\right)=\frac{\zeta^{z}\left(v, v_{+}\right)}{\zeta^{z}\left(v_{+}, v\right)} G^{z}(v, v)=\left(\zeta^{z}\left(v, v_{+}\right)+\frac{S_{z}\left(L_{v}\right)}{G^{z}(v, v)}\right) \zeta^{z}\left(v, v_{+}\right) G^{z}(v, v)
$$


as claimed. Using Proposition 3.1, we thus deduce the existence of $G^{\lambda}(w, w)$ for all $w \in \mathbb{T}_{b_{o}}^{+}$. Similarly the existence of $w \in \mathbb{T}_{b_{o}}^{-}$follows from the analog of Proposition 3.1 with the $\left(N_{i, j}\right)$ system. Finally using (2.13) we see that (a) implies $G^{\lambda}(v, v) \neq 0$.

We now observe that under $(\mathbf{C 1} *)$, all the WT functions are related as follows:

Lemma 3.3. Suppose $\mathbb{T}$ satisfies $(\boldsymbol{C 1} *)$ and let $\lambda \in \mathbb{R} \backslash\left(\mathfrak{D} \cup \sigma_{D}\right)$.

(i) If $\operatorname{Im} R_{\lambda+\mathrm{i} 0}^{ \pm}(j)=0$ for some $j \in \mathfrak{A}^{ \pm}$, then $\operatorname{Im} R_{\lambda+\mathrm{i} 0}^{ \pm}(j)=0$ for all $j \in \mathfrak{A}^{ \pm}$.

(ii) Assume $\lambda \in \mathbb{R} \backslash \mathfrak{D}_{0}$.

If $\operatorname{Im} R_{\lambda+\mathrm{i} 0}^{+}(j)=0$ for some $j \in \mathfrak{A}^{+}$and $\operatorname{Im} R_{\lambda+\mathrm{i} 0}^{-}(k)=0$ for some $k \in \mathfrak{A}^{-}$, then $\operatorname{Im} G^{\lambda+\mathrm{i} 0}(v, v)=0$ and $\operatorname{Im} R_{\lambda+\mathrm{i} 0}^{ \pm}(v)=0$ for all $v \in \mathbb{T}$.

The same conclusion holds if $\operatorname{Im} G^{\lambda+\mathrm{i} 0}(w, w)=0$ for some $w \in \mathbb{T}$.

(iii) For any $v \in \mathbb{T}$, we have

$$
\sigma\left(\mathscr{H}_{0}\right) \backslash \mathfrak{D}_{0}=\overline{\left\{\lambda \in \mathbb{R} \backslash \mathfrak{D}_{0}: \operatorname{Im} G^{\lambda+\mathrm{i} 0}(v, v)>0\right\}} \backslash \mathfrak{D}_{0} .
$$

Proof. We first note that $\zeta_{j}^{\lambda} \neq 0$, due to the relation

$$
\zeta_{j}^{\lambda}=\frac{h_{j}}{S_{\lambda}\left(L_{j}\right)}=\frac{-1 / S_{\lambda}\left(L_{j}\right)}{\sum_{k=1}^{m} \frac{M_{j, k}}{S_{\lambda}^{2}\left(L_{k}\right)} h_{k}-F_{j}(\lambda)}
$$

and the fact that the $h_{k}$ are finite.

Suppose that $\operatorname{Im} R_{\lambda}^{+}(k)>0$ for some $k \in \mathfrak{A}^{+}$and let $l \in \mathfrak{A}^{+}$. Then by $(\mathbf{C 1} *)$, $\left(M^{n}\right)_{l, k} \geq 1$, so if $v$ has label $l$ and $w$ has label $k$, there is a path $\left(u_{0}, \ldots, u_{r}\right)$ with $u_{1}=v$ and $u_{r}=w$. Denote $b_{j}=\left(u_{j-1}, u_{j}\right)$. Then applying (2.17) repeatedly,

$$
\begin{aligned}
\operatorname{Im} R_{\lambda}^{+}(l)=\operatorname{Im} R_{\lambda}^{+}\left(o_{b_{1}}\right) & \geq \sum_{\left(e_{2} ; e_{r}\right)}\left|\zeta^{\lambda}\left(e_{1}\right) \cdots \zeta^{\lambda}\left(e_{r-1}\right)\right|^{2} \operatorname{Im} R_{\lambda}^{+}\left(o_{e_{r}}\right) \\
& \geq\left|\zeta^{\lambda}\left(b_{1}\right) \cdots \zeta^{\lambda}\left(b_{r-1}\right)\right|^{2} \operatorname{Im} R_{\lambda}^{+}\left(o_{b_{r}}\right)>0
\end{aligned}
$$

where the sum runs over all $(r-1)$-paths $\left(e_{2} ; e_{r}\right)$ outgoing from $b_{1}$, and the last inequality holds because $\operatorname{Im} R_{\lambda}^{+}\left(o_{b_{r}}\right)=\operatorname{Im} R_{\lambda}^{+}(k)>0$ and all $\zeta_{j}^{\lambda} \neq 0$.

So under $(\mathbf{C 1} *)$, if $\operatorname{Im} R_{\lambda}^{+}(j)>0$ for some $j \in \mathfrak{A}^{+}$, then $\operatorname{Im} R_{\lambda}^{+}(k)>0$ for all $k \in \mathfrak{A}^{+}$. So if $\operatorname{Im} R_{\lambda}^{+}(j)=0$ for some $j \in \mathfrak{A}^{+}$, then it must be zero for all $j \in \mathfrak{A}^{+}$.

The proof for $R_{\lambda+\mathrm{i} 0}^{-}$is the same.

For (ii), say $t_{b_{o}}$ has type $j_{o}$. Since $R_{\lambda}^{+}\left(t_{b_{o}}\right)=\sum_{k=1}^{m} M_{j_{o}, k} R_{\lambda}^{+}(k)-\alpha_{t_{b_{o}}}$ by (2.12), we get $\operatorname{Im} R_{\lambda}^{+}\left(t_{b_{o}}\right)=0$ by (i). Using (2.5), this implies $\operatorname{Im} \zeta^{\lambda}\left(b_{o}\right)=0$, which by (2.4) implies that $\operatorname{Im} R_{\lambda}^{+}\left(o_{b_{o}}\right)=0$. Similarly, if $o_{b_{o}}$ has type $j$, then $R_{\lambda}^{-}\left(o_{b_{o}}\right)=$ $\sum_{k=1}^{n} N_{j, k} R_{\lambda}^{-}(k)+\alpha_{o_{b_{o}}}$, so $\operatorname{Im} R_{\lambda}^{-}\left(o_{b_{o}}\right)=0$ by (i), also implying $\operatorname{Im} R_{\lambda}^{-}\left(t_{b_{o}}\right)=0$ via (2.5), (2.4). Now $R_{\lambda}^{+}(v)+R_{\lambda}^{-}(v) \neq 0$ for $v=o_{b_{o}}, t_{b_{o}}$. Using (2.13), this implies $G^{\lambda}(v, v)$ exists, and $\operatorname{Im} G^{\lambda}(v, v)=0$ for $v=o_{b_{o}}, t_{b_{o}}$. Since $\operatorname{Im} G^{\lambda}\left(t_{b_{o}}, t_{b_{o}}\right)=0$ and $\operatorname{Im} \zeta^{\lambda}\left(t_{b_{o}}, v_{+}\right)=\operatorname{Im} \zeta_{j}^{\lambda}=0$, then using (3.7), we get $\operatorname{Im} G^{\lambda}\left(v_{+}, v_{+}\right)=0$ for any $v_{+} \in \mathscr{N}_{t_{b_{o}}}^{+}$, so $\operatorname{Im} G^{\lambda}(w, w)=0$ for all $w \in \mathbb{T}_{b_{o}}^{+}$by induction. Similarly, we may use $\operatorname{Im} G^{\lambda}\left(o_{b_{o}}, o_{b_{o}}\right)=0$ along with (2.7) to deduce that $\operatorname{Im} G^{\lambda}(v, v)=0$ for all $v \in \mathbb{T}_{b_{o}}^{-}$. This proves claim (ii) for the Green function.

Next, if $v \in \mathbb{T}_{b_{o}}^{+}$, we know that $\operatorname{Im} R_{\lambda}^{+}(v)=\operatorname{Im} R_{\lambda}^{+}(j)=0$. By Lemma $3.2 G^{\lambda}(v, v)$ exists, and we showed $\operatorname{Im} G^{\lambda}(v, v)=0$. Using (2.13), it follows that $\operatorname{Im} R_{\lambda}^{-}(v)=0$. Hence, $\operatorname{Im} R_{\lambda}^{ \pm}(v)=0$ for $v \in \mathbb{T}_{b_{o}}^{+}$. The claim for $\mathbb{T}_{b_{o}}^{-}$follows similarly. 
Now suppose that $\operatorname{Im} G^{\lambda}(w, w)=0$ for some $w \in \mathbb{T}$. By symmetry we may assume $w \in \mathbb{T}_{b_{o}}^{+}$. Recall that $G^{\lambda}(v, v), R_{\lambda}^{ \pm}(v)$ exist by Lemma 3.2. By (2.13), we get $\operatorname{Im} R_{\lambda}^{+}(w)=\operatorname{Im} R_{\lambda}^{-}(w)=0$. Consider $w_{+} \in \mathscr{N}_{w}^{+}$. By Proposition 3.1, $\zeta^{\lambda}\left(w, w_{+}\right)$exists, so using (2.4), we get $\operatorname{Im} \zeta^{\lambda}\left(w, w_{+}\right)=0$, hence $\operatorname{Im} G^{\lambda}\left(w_{+}, w_{+}\right)=0$. On the other hand $\operatorname{Im} R_{\lambda}^{+}(w)=0$ implies $\operatorname{Im} R_{\lambda}^{+}\left(w_{-}\right)=0$ by (i), so we similarly get $\operatorname{Im} \zeta^{\lambda}\left(w_{-}, w\right)=0$ and $\operatorname{Im} G^{\lambda}\left(w_{-}, w_{-}\right)=0$. This shows that $\operatorname{Im} G^{\lambda}(v, v)=0$ for all $v \in \mathbb{T}_{b_{o}}^{+}$and also for $v=o_{b_{o}}$, since by definition (3.6), $\operatorname{Im} R_{\lambda}^{+}\left(o_{b_{o}}\right)=: \operatorname{Im} R_{\lambda}^{+}\left(j_{o}\right)=0$ for $v=t_{b_{o}}$, if $\ell\left(t_{b_{o}}\right)=j_{o}$. Now if $u \in \mathscr{N}_{o_{b_{o}}}^{-}$, then as in (3.7) we have $G^{\lambda}(u, u)=S_{\lambda}\left(L_{u}\right) \zeta^{\lambda}\left(o_{b_{o}}, u\right)+$ $\zeta^{\lambda}\left(o_{b_{o}}, u\right)^{2} G^{\lambda}\left(o_{b_{o}}, o_{b_{o}}\right)$, but $\operatorname{Im} R_{\lambda}^{-}\left(o_{b_{o}}\right)=0$ implies we have $\sum_{b^{-} \in \mathscr{N}_{b_{o}}^{-}} \operatorname{Im} R_{\lambda}^{-}\left(t_{b^{-}}\right)=$ $\operatorname{Im} R_{\lambda}^{-}\left(o_{b_{o}}\right)=0$, so $\operatorname{Im} R_{\lambda}^{-}\left(t_{b^{-}}\right)=0$ for each $b^{-}$, so $\operatorname{Im} R_{\lambda}^{-}(k)=0$ for all $k \in \mathfrak{A}^{-}$by (i) and we deduce again that $\operatorname{Im} G^{\lambda}(u, u)=0$ for $u \in \mathscr{N}_{o_{b_{o}}}^{-}$, hence for all $u \in \mathbb{T}_{b_{o}}^{-}$by induction.

Finally, to prove (iii), recall that if $E_{\mathscr{H}_{0}}$ is the projection-valued measure $E_{\mathscr{H}_{0}}(J)=$ $\chi_{J}\left(\mathscr{H}_{0}\right)$, then $\sigma\left(\mathscr{H}_{0}\right)=\operatorname{supp} E_{\mathscr{H}_{0}}$. Moreover, $E_{\mathscr{H}_{0}}(J)=0$ if and only if $\mu_{f}(J)=0$ for all $f \in L^{2}(\mathbf{T})$, where $\mu_{f}(J)=\left\langle f, \chi_{J}\left(\mathscr{H}_{0}\right) f\right\rangle$. By [32, Lemma 3.13], we know that supp $\mu_{f}=\overline{\left\{\lambda \in \mathbb{R}: \operatorname{Im}\left\langle f, G^{\lambda} f\right\rangle>0\right\}}$. Since $\mathfrak{D}_{0}$ is a discrete set, we deduce that $\operatorname{supp} \mu_{f} \backslash \mathfrak{D}_{0}=\overline{\left\{\lambda \in \mathbb{R} \backslash \mathfrak{D}_{0}: \operatorname{Im}\left\langle f, G^{\lambda} f\right\rangle>0\right\}} \backslash \mathfrak{D}_{0}$.

Let $\lambda_{0} \in \mathbb{R} \backslash \mathfrak{D}_{0}$ and suppose there is $\varepsilon$ such that $\operatorname{Im} G^{\lambda}(v, v)=0$ for all $I:=$ $\left(\lambda_{0}-\varepsilon, \lambda_{0}+\varepsilon\right)$. Then $\operatorname{Im} R_{\lambda}^{ \pm}(w)=0$ for all $w$ by (ii), $\operatorname{so} \operatorname{Im}\left\langle f, G^{\lambda} f\right\rangle=0$ for any $f$ by Lemma A.1. Thus, $\mu_{f}(I)=0$ for all $f \in L^{2}(\mathbf{T})$, so $E_{\mathscr{H}_{0}}(I)=0$ and thus $\lambda_{0} \notin \sigma\left(\mathscr{H}_{0}\right)$.

Conversely, fix $\lambda \in \mathbb{R} \backslash \mathfrak{D}_{0}$ and $v \in \mathbb{T}$. If $\operatorname{Im} G^{\lambda}(v, v)>0$, then $\operatorname{Im} R_{\lambda}^{+}(v)>0$ or $\operatorname{Im} R_{\lambda}^{-}(v)>0$, say the former holds and let $v=o_{e}$. Then by Lemma A. $1, \operatorname{Im}\left\langle f, G^{\lambda} f\right\rangle>$ 0 for $f=\phi_{\lambda ; e}^{-}$, since $g_{\phi_{\lambda ; e}^{-}}^{-}(\lambda) \geq \frac{1}{6}\left\|\phi_{\lambda ; e}^{-}\right\|^{4}>0$. In fact, $\left\|\phi_{\lambda ; e}^{-}\right\|=0$ would imply $C_{\lambda}(x)=R_{\lambda}^{-}\left(o_{e}\right) S_{\lambda}(x)$ for all $x \in e$, contradicting the fact that $C_{\lambda}$ and $S_{\lambda}$ are linearly independent. We thus showed that $\overline{\left\{\lambda \in \mathbb{R} \backslash \mathfrak{D}_{0}: \operatorname{Im} G^{\lambda}(v, v)>0\right\}} \backslash \mathfrak{D}_{0} \subseteq \operatorname{supp} \mu_{f} \backslash \mathfrak{D}_{0} \subseteq$ $\sigma\left(\mathscr{H}_{0}\right) \backslash \mathfrak{D}_{0}$.

Lemma 3.4. If $\mathbb{T}$ satisfies (C1*), then:

(i) For any $j \in \mathfrak{A}^{+}, k \in \mathfrak{A}^{-}$, the map $\sigma(\mathscr{H}) \backslash \mathfrak{D}_{0} \ni \lambda \mapsto \operatorname{Im} R_{\lambda+\mathrm{i} 0}^{+}(j)+\operatorname{Im} R_{\lambda+\mathrm{i} 0}^{-}(k)$ has a discrete set of zeroes. The same holds for $\sigma(\mathscr{H}) \backslash \mathfrak{D}_{0} \ni \lambda \mapsto \operatorname{Im} G^{\lambda+\mathrm{i} 0}(v, v)$, for any $v \in \mathbb{T}$.

(ii) $\sigma(\mathscr{H})$ is a union of closed intervals and isolated points, $\bigcup_{r} I_{r} \cup \mathfrak{P}$. The limits $G^{\lambda+\mathrm{i} 0}(v, v)$ exist in the interior $\stackrel{\circ}{I}_{r}$ and satisfy $\operatorname{Im} G^{\lambda+\mathrm{i} 0}(v, v)>0$, for any $v \in \mathbb{T}$.

(iii) The spectrum of $H_{\mathrm{T}}$ is purely absolutely continuous in any compact subset $K \subset \stackrel{\circ}{I}_{r}$.

Proof. We know that $f(\lambda)=R_{\lambda}^{+}(j)+R_{\lambda}^{-}(k)$ is analytic on $\mathbb{R} \backslash \mathfrak{D}_{0}$, so if $\operatorname{Im} f\left(\lambda_{0}\right)=0$ for some $\lambda_{0} \notin \mathfrak{D}_{0}$, we may expand $\operatorname{Im} f(\lambda)=\sum_{n \geq 0} b_{n}\left(\lambda-\lambda_{0}\right)^{n}$ for $\lambda \in\left(\lambda_{0}-\varepsilon, \lambda_{0}+\varepsilon\right)$, where $b_{n}=\operatorname{Im} a_{n}$ and $\left(a_{n}\right)$ are the coefficients for $f(\lambda)$. Suppose $\lambda_{0} \in \sigma(\mathscr{H}) \backslash \mathfrak{D}_{0}$. If all $b_{n}=0$ then $\operatorname{Im} f$ is identically zero on $\left(\lambda_{0}-\varepsilon, \lambda_{0}+\varepsilon\right)$. In view of Lemma 3.3 (ii)-(iii), this contradicts that $\lambda_{0} \in \sigma(\mathscr{H})$. Hence let $k$ be the smallest index with $b_{k} \neq 0$. Then $\operatorname{Im} f(\lambda)=\left(\lambda-\lambda_{0}\right)^{k} g(\lambda)$, where $g(\lambda)=\sum_{n \geq 0} b_{n+k}\left(\lambda-\lambda_{0}\right)^{n}$. Clearly $g\left(\lambda_{0}\right)=b_{k} \neq 0$ and $g$ is continuous, so we may find $\varepsilon^{\prime} \leq \varepsilon$ such that both $\left(\lambda-\lambda_{0}\right)^{k}$ and $g(\lambda)$ are nonzero on $\left(\lambda_{0}-\varepsilon^{\prime}, \lambda_{0}+\varepsilon^{\prime}\right) \backslash\left\{\lambda_{0}\right\}$. This shows that $\lambda_{0}$ is an isolated zero of $\operatorname{Im} f$, as required.

This proves the first part of (i). For the second part, suppose $\operatorname{Im} G^{\lambda}(w, w)=0$ for some $w \in \mathbb{T}$. By Lemma 3.3, this implies $\operatorname{Im} R_{\lambda}^{+}(j)+\operatorname{Im} R_{\lambda}^{-}(k)=0$. Hence, $\lambda$ must lie in the preceding discrete set of zeroes. 
For (ii), recall that $\sigma\left(\mathscr{H}_{0}\right) \backslash \mathfrak{D}_{0}=\overline{\left\{\lambda \in \mathbb{R} \backslash \mathfrak{D}_{0}: \operatorname{Im} G^{\lambda}(v, v)>0\right\}} \backslash \mathfrak{D}_{0}$, again by Lemma 3.3. By Proposition 3.1 and (2.4), we know $\lambda \mapsto R_{\lambda}^{ \pm}(v)$ is continuous, so $\mathbb{R} \backslash \mathfrak{D}_{0} \ni \lambda \mapsto G^{\lambda}(v, v)$ is continuous by (2.13). Hence, $\left\{\lambda \in \mathbb{R} \backslash \mathfrak{D}_{0}: \operatorname{Im} G^{\lambda}(v, v)>0\right\}$ is a union of intervals $\bigcup_{r} J_{r}$ which is independent of $v$ by Lemma 3.3. We take $I_{r}$ as the closure of $J_{r}$ and $\mathfrak{P}=\sigma(H) \cap \mathfrak{D}_{0}$.

Finally, if $K$ is a compact subset of $\check{I}_{r}$, we know that $G^{\lambda}(v, v)$ is uniformly bounded, and the same holds for $R_{\lambda}^{ \pm}(v)$. In particular, if $v=o_{e}$ and $\psi$ is supported in $e$, we get using respresentation (A.2) along with (2.13) that $\sup _{\lambda \in K}\left|\left\langle\psi, G^{\lambda} \psi\right\rangle\right|<\infty$. The claim follows by the density of the linear span of such $\psi$.

This completes the proof of Theorem 1.1. We next move to Theorem 1.2.

Remark 3.2. Condition (C1) implies (C1*). In fact, as remarked in [5], all cone types are indexed by the directed edges of the finite graph $\mathbf{G}$. If we consider the universal cover T rooted at the midpoint $o$ of some $b_{o} \in B(\mathbf{G})$ (here $o$ is not viewed as an added vertex, just a reference point), this means that the type of each vertex $v \in \mathbb{T}$ is determined by a directed edge, so there are at most $|B(\mathbf{G})|$ types. By [25, Lemma 3.1], we know the non-backtracking matrix of $B(\mathbf{G})$ is irreducible. This implies that if $\mathbf{T}$ is considered in the twisted view, and if $M$ is the single matrix over some alphabet $\mathfrak{A}$ encoding all cone types, then $M$ satisfies: for any $k, l \in \mathfrak{A}$, there is $n(k, l)$ such that $\left(M^{n}\right)_{k, l} \geq 1$. In particular, $\left(\mathbf{C} 1^{*}\right)$ holds if we take the matrices $\tilde{M}, N$ encoding the types in $\mathbb{T}_{b_{o}}^{+}$and $\mathbb{T}_{b_{o}}^{-}$, respectively.

Proof of Theorem 1.2. Since (C1) implies (C1*), we already know that $\sigma\left(\mathscr{H}_{0}\right)$ has the structure given in Theorem 1.1. Let $\lambda \in \stackrel{I}{I}_{r}$ be in the interior of an AC band.

Within the twisted view, all vertices are offspring of $o$ and we deal with the single, combined alphabet $\mathfrak{A}$. Under the stronger assumption $(\mathbf{C 1})$, we know the larger matrix $M$ is irreducible. Consequently, if we suppose that $\operatorname{Im} R_{\lambda+i 0}^{+}(j)=0$ for some $j \in \mathfrak{A}$, then the statement in Lemma 3.3 (i) now implies that $\operatorname{Im} R_{\lambda+\mathrm{i} 0}^{+}(j)=0$ for all $j \in \mathfrak{A}$.

Now let $v \in \mathbb{T}$. We know $\operatorname{Im} G^{\lambda}(v, v)>0$, so by (2.13), $\operatorname{Im} R_{\lambda}^{+}(v)+\operatorname{Im} R_{\lambda}^{-}(v)>0$, so either $\operatorname{Im} R_{\lambda}^{+}(v)>0$ or $\operatorname{Im} R_{\lambda}^{-}(v)>0$ by the Herglotz property. In the former case we are done. Suppose that $\operatorname{Im} R_{\lambda}^{+}(v)=0$. Say $v=t_{b}$ for some $b \in B(\mathbb{T})$ and $\ell(v)=j$. Then $0=\operatorname{Im} R_{\lambda}^{+}\left(t_{b}\right)=\sum_{k=1}^{m} M_{j, k} \operatorname{Im} R_{\lambda}^{+}(k)$ implies that $\operatorname{Im} R_{\lambda}^{+}(k)=0$ for some, hence all, $k \in \mathfrak{A}$. But by (2.4), $R_{\lambda}^{-}\left(t_{b}\right)=R_{\lambda}^{+}\left(o_{\widehat{b}}\right)+\frac{C_{\lambda}\left(L_{b}\right)-S_{\lambda}^{\prime}\left(L_{b}\right)}{S_{\lambda}\left(L_{b}\right)}$, so $\operatorname{Im} R_{\lambda}^{-}\left(t_{b}\right)=\operatorname{Im} R_{\lambda}^{+}\left(o_{\widehat{b}}\right)$. As mentioned in Remark 3.2, under (C1), all cone types are indexed by the directed edges of $\mathbf{G}$, in particular $\mathbb{T}_{\widehat{b}}^{+}$is one of the finitely many nonisomorphic cones ${ }^{5}$. In other words, $\operatorname{Im} R_{\lambda}^{+}\left(o_{\widehat{b}}\right)=\operatorname{Im} R_{\lambda}^{+}(r)$ for some $r \in \mathfrak{A}$. Hence, $\operatorname{Im} R_{\lambda}^{-}\left(t_{b}\right)=0$. We thus get $\operatorname{Im} R_{\lambda}^{+}(v)+\operatorname{Im} R_{\lambda}^{-}(v)=0$, a contradiction. Thus, $\operatorname{Im} R_{\lambda}^{+}(v)>0$.

\section{Examples of Nontrivial Spectrum}

For Theorem 1.2 to be interesting, we'll need to know that $\sigma\left(\mathscr{H}_{0}\right)$ is not reduced to the isolated points $\mathfrak{P}$. Our aim in this section is to give some examples in which this can be proved. We believe the phenomenon to be true for a wider class of examples.

\footnotetext{
5 This property is why we need (C1); it is not necessarily true under (C1*). cf. footnote in Sect. 1.2.
} 
4.1. Equilateral trees. Let $G$ be a discrete graph of minimal degree $\geq 2$ and $\mathbb{T}=\widetilde{G}$ its universal cover. We know from [10, Sect. 1.6] that the spectrum of the adjacency matrix $\sigma\left(\mathscr{A}_{\mathbb{T}}\right)$ has a continuous part. Actually their argument remains valid for the normalized adjacency matrix $P f(x)=\frac{1}{d(x)}(\mathscr{A} f)(x)$ (and also if we add potentials). Consequently, using [11, Theorem 3.18], the induced quantum tree with equilateral edge length, identical symmetric potentials, and identical coupling constants, will also have some continuous spectrum. Using Theorem 1.2, we can now conclude:

If $G$ is a graph of degree $\geq 2$, if $\mathbb{T}=\widetilde{G}$ is its universal cover, and we endow each edge of $\mathbb{T}$ with the same length $L$ and potential $W$, and each vertex with the same coupling constant $\alpha$, then $\sigma\left(\mathscr{H}_{\mathbf{T}}\right)$ consists of non-empty bands of purely absolutely continuous spectrum, and possibly some isolated eigenvalues.

This generalizes the case of regular trees previously considered in [12,31].

We may easily extend this to graphs with several lengths which are rationally dependent. More precisely, if in $\mathbf{G}$, we have $L_{j}=n_{j} L$ for some $n_{j} \in \mathbb{N}^{*}$, add $n_{j}$ vertices of degree 2 to the edge $e_{j}$, with Kirchhoff-Neumann conditions. Then using the previous claim, we see that $\mathbf{T}$ also has nontrivial AC spectrum in this case.

4.2. An argument of Bordenave-Sen-Virág. We now consider the non-equilateral case. For this, we start by adapting an argument from [10] to quantum graphs.

We begin with some definitions, which appear in a more general framework in [10]. Let $G$ be a discrete graph and $\mathbb{T}=\widetilde{G}$ its universal cover.

A labeling (or colouring) of the vertices of $\mathbb{T}$ is a map $\eta: V(\mathbb{T}) \rightarrow \mathbb{Z}$. With respect to a given labeling, we call a vertex $v$ :

(a) prodigy if it has a neighbour $w$ with $\eta(w)<\eta(v)$ and such that all other neighbours of $w$ also have label less than $\eta(v)$,

(b) level if it is not a prodigy and if all of its neighbours have the same or lower labels,

(c) bad if it is neither prodigy nor level.

The tree $\mathbb{T}=\widetilde{G}$ is equipped with a natural unimodular ${ }^{6}$ measure on the space of rooted graphs, namely $\mathbb{P}=\frac{1}{|G|} \sum_{x \in G} \delta_{[\widetilde{G}, \tilde{x}]}$.

We say the labeling $\eta$ on $\mathbb{T}$ is invariant if there exists a unimodular probability measure on the set of coloured rooted graphs, which is concentrated on $\{[\mathbb{T}, v, \eta]\}_{v \in \mathbb{T}}$. See e.g. [3, Appendix A] for some background on coloured rooted graphs.

Let $S \subset \ell^{2}(\widetilde{G})$ be a subspace and let $P_{S}$ be the orthogonal projection onto $S$. We say that $S$ is invariant if $P_{S}(g v, g w)=P_{S}(v, w)$ for any $g \in \Gamma$, where $\Gamma$ is the group of covering transformations with $\widetilde{G} / \Gamma \equiv G$.

Given an invariant subspace $S \subset \ell^{2}(\widetilde{G})$, we define its von-Neumann dimension by

$$
\operatorname{dim}_{\mathrm{VN}} S=\mathbb{E}_{\mathbb{P}}\left[\left\langle\delta_{o}, P_{S} \delta_{o}\right\rangle\right]=\frac{1}{|G|} \sum_{x \in G} P_{S}(\tilde{x}, \tilde{x}) .
$$

A line ensemble in $\mathbb{T}$ is a disjoint union of bi-infinite lines $\left(l_{i}\right)$. More precisely, $\mathscr{L}: V(\mathbb{T}) \times V(\mathbb{T}) \rightarrow\{0,1\}$ is a line ensemble if:

$-\mathscr{L}(u, v)=0$ if $\{u, v\} \notin E(\mathbb{T})$,

- $\mathscr{L}(u, v)=\mathscr{L}(v, u)$,

6 A measure is unimodular if it invariant under the moving of the root. More precisely, it should satisfy $\int \sum_{o^{\prime} \sim o} f\left(\left[G, o, o^{\prime}\right]\right) \mathrm{d} \mathbb{P}([G, o])=\int \sum_{o^{\prime} \sim o} f\left(\left[G, o^{\prime}, o\right]\right) \mathrm{d} \mathbb{P}([G, o])$. See $[10$, Sect. 1.4] for details. 


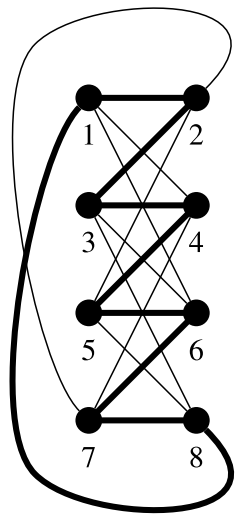

Hamiltonian cycle in $K_{4,4}$.

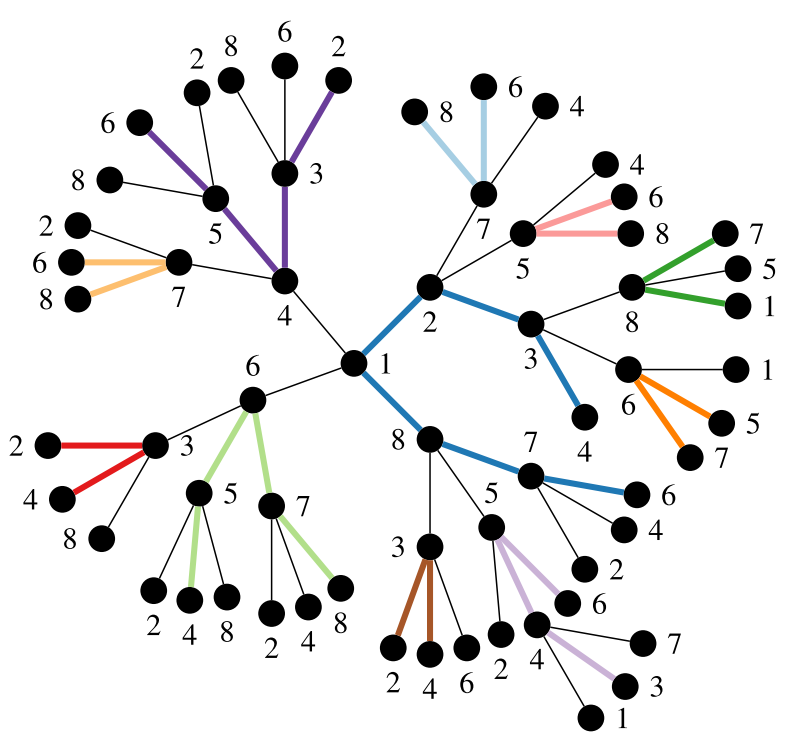

Fig. 2. The lift of a Hamiltonian cycle is a line ensemble covering all vertices of $\mathbb{T}$. (Each coloured bold line is infinite.)

- for any $v \in V(\mathbb{T})$, we have $\sum_{u} \mathscr{L}(u, v) \in\{0,2\}$.

Abusing notation, we then let $\mathscr{L}=\{e: \mathscr{L}(e)=1\}$, which gives a subgraph consisting of disjoint lines.

We say a line ensemble $\mathscr{L}$ is invariant if there exists a unimodular probability measure on the space of weighted rooted graphs, which is concentrated on the collection $\{[\mathbb{T}, v, \mathscr{L}]\}_{v \in \mathbb{T}}$.

We say that $\mathbb{T}$ is Hamiltonian if there exists an invariant line ensemble $\mathscr{L}$ that contains the root with probability 1 .

Remark 4.1. Recall that a finite graph $G$ is Hamiltonian if there is a cycle in $G$ which visits each vertex exactly once. If $G$ is Hamiltonian, then $\widetilde{G}$ is Hamiltonian in the above sense. In fact, if $C=\left(x_{0}, \ldots, x_{m}\right)$ is a cycle in $G$, then its lift to $\widetilde{G}$ is a line ensemble $\mathscr{L}$ which generally consists of a disjoint union of countable lines $\left(l_{i}\right)$, where $l_{i}=\left(\ldots, \tilde{x}_{0}, \ldots, \tilde{x}_{m}, \tilde{x}_{0}, \ldots\right)$ (see Fig. 2). Since it is a lift, this line ensemble is invariant. More precisely, if $[H, v, R]$ denotes an equivalence class of graph $H$ with root $v$ and edge weight $R(e)$ for $e \in E(H)$, then $[\widetilde{G}, v, \mathscr{L}]=[\widetilde{G}, g v, \mathscr{L}]$ for any covering transformation $g$. This by definition of the universal cover and $\mathscr{L}$. So the measure $\tilde{\mathbb{P}}=\frac{1}{|G|} \sum_{x \in G} \delta_{[\widetilde{G}, \tilde{x}, \mathscr{L}]}$ is well-defined and unimodularity follows from $\sum_{(x, y) \in B(G)} f(x, y)=\sum_{(x, y) \in B(G)} f(y, x)$.

Moreover, $\tilde{\mathbb{P}}(o \in \mathscr{L})=\frac{1}{|G|} \sum_{x \in G} 1_{\tilde{x} \in \mathscr{L}}=\frac{1}{|G|} \sum_{x \in G} 1_{x \in C}=\frac{|C|}{|G|}$. If $C$ covers $G$, we thus get $\tilde{\mathbb{P}}(o \in \mathscr{L})=1$.

In particular, the $(q+1)$-regular tree $\mathbb{T}_{q}$ is Hamiltonian, since it covers the complete bipartite $(q+1)$-regular graph on $2(q+1)$ vertices, which is Hamiltonian.

We may now state our adaptation of [10, Theorem 1.5] to quantum trees. Here, if $G$ is a finite graph, we denote by $\mathbf{G}=\mathbf{G}(\alpha, \mathbb{L}, \mathbb{W})$ the quantum graph obtained by endowing 
each edge with a length $L_{e}$, a potential $W_{e}$ and each vertex a coupling constant $\alpha_{v}$, so the Schrödinger operator $\mathscr{H}=-\Delta+W$ acts with $\delta$-conditions. We say that $\mathbf{T}=\widetilde{\mathbf{G}}$ if $\mathbb{T}=\widetilde{G}$ is endowed with the lifted structure $\alpha_{v}=\alpha_{\pi v}, L_{(u, v)}=L_{(\pi u, \pi v)}$ and $W_{(u, v)}=W_{(\pi u, \pi v)}$.

Recall definition (1.5) of $S_{z}\left(x_{b}\right)$.

Proposition 4.1. Suppose $G$ is a finite Hamiltonian graph. Endow $G$ with a quantum structure $\mathbf{G}$ with $\delta$-conditions and let $\mathbf{T}=\widetilde{\mathbf{G}}$. If $\lambda$ is an eigenvalue of $\mathscr{H}_{\mathbf{T}}$, then $\lambda$ must be a Dirichlet value, i.e. $S_{\lambda}\left(L_{b}\right)=0$ for some $b$.

Proof. Suppose $\mathscr{H}_{\mathbf{T}}$ has an eigenvalue, say $\mathscr{H}_{\mathbf{T}} \varphi_{\lambda}=\lambda \varphi_{\lambda}$ for some $\varphi_{\lambda} \in L^{2}(\mathscr{T})$.

Suppose on the contrary that $S_{\lambda}\left(L_{b}\right) \neq 0$ for all $b$.

Let $\stackrel{\circ}{\varphi}=\left.\varphi\right|_{V}$. We claim that

$$
\left(\mathscr{A}_{\lambda} \dot{\circ}_{\lambda}\right)(v)=W_{\lambda}(v) \dot{\circ}_{\lambda}(v),
$$

where

$$
\left(\mathscr{A}_{\lambda} \psi\right)(v)=\sum_{u \sim v} \frac{\psi(u)}{S_{\lambda}\left(L_{u v}\right)} \quad \text { and } \quad W_{\lambda}(v)=\alpha_{v}+\sum_{u \sim v} \frac{C_{\lambda}\left(L_{u v}\right)}{S_{\lambda}\left(L_{u v}\right)}
$$

and $u v:=(u, v)$. In fact,

$$
\begin{aligned}
\sum_{u \sim v} \frac{\varphi_{\lambda}(u)}{S_{\lambda}\left(L_{v u}\right)}=\sum_{u \sim v} \frac{\varphi_{\lambda}\left(t_{v u}\right)}{S_{\lambda}\left(L_{v u}\right)} & =\sum_{u \sim v}\left[\frac{\varphi_{\lambda}\left(o_{v u}\right) C_{\lambda}\left(L_{v u}\right)}{S_{\lambda}\left(L_{v u}\right)}+\varphi_{\lambda}^{\prime}\left(o_{v u}\right)\right] \\
& =\varphi_{\lambda}(v) \sum_{u \sim v} \frac{C_{\lambda}\left(L_{v u}\right)}{S_{\lambda}\left(L_{v u}\right)}+\alpha_{v} \varphi_{\lambda}(v) .
\end{aligned}
$$

Let $\mathfrak{E}_{\lambda} \subset \ell^{2}(\mathbb{T})$ be the set of functions satisfying this eigenvalue equation, i.e. $\psi \in \mathfrak{E}_{\lambda}$ if and only if

$$
W_{\lambda}(w) \psi(w)=\sum_{u \sim w} \frac{\psi(u)}{S_{\lambda}\left(L_{u w}\right)}
$$

Note that $\mathfrak{E}_{\lambda}$ is invariant. In fact, let $M_{\lambda}=\mathscr{A}_{\lambda}-W_{\lambda}$. Then $M_{\lambda}$ is self-adjoint. This is because all weights are real-valued and symmetric. Moreover, $\mathfrak{E}_{\lambda}=\operatorname{ker} M_{\lambda}$. Now, if $g \in \Gamma$, let $\left(U_{g} f\right)(v)=f\left(g^{-1} v\right)$. Using that $\alpha_{g v}=\alpha_{v} L_{(g u, g v)}=L_{(u, v)}$, $W_{(g u, g v)}=W_{(u, v)}$, it easily follows that $U_{g}^{-1} M_{\lambda} U_{g}=M_{\lambda}$. Standard arguments imply that $U_{g}^{-1} P_{\mathfrak{E}_{\lambda}} U_{g}=P_{\mathfrak{E}_{\lambda}}$, so $\mathfrak{E}_{\lambda}$ is indeed invariant.

Let $C$ be a Hamiltonian cycle in $G$, so its lift $\mathscr{L}$ is a line ensemble as in Remark 4.1. Using the line ensemble, we may use the construction of [10, Theorem 1.5] to define for any $k \in \mathbb{N}^{*}$ an invariant labeling $\eta_{k}: V(\mathbb{T}) \rightarrow \mathbb{Z}_{k}$ of the vertices of $\mathbb{T}$ by integers which satisfies:

$-b:=\mathbb{P}(o$ is bad $) \leq 1 / k$,

- vertices in $\mathscr{L}$ with $\eta_{k}(v) \neq 0$ are prodigy. Vertices in $\mathscr{L}$ with $\eta_{k}(v)=0$ are bad,

- vertices outside $\mathscr{L}$ are level. 
In our case, all vertices are in $\mathscr{L}$, so there are no level vertices.

We now argue as in [10, Theorem 2.3]. Here the situation is simpler as there are no level vertices. Let $\mathfrak{B}$ be the space of vectors which vanish on the set of bad vertices. Then $\operatorname{dim}_{\mathrm{VN}} \mathfrak{B}=\mathbb{P}(o$ is not bad $)=1-b$. Let $\mathfrak{E}^{\prime}=\mathfrak{E}_{\lambda} \cap \mathfrak{B}$. Then using $\operatorname{dim}_{\mathrm{VN}}(R \cap Q) \geq$ $\operatorname{dim}_{\mathrm{VN}} R+\operatorname{dim}_{\mathrm{VN}} Q-1$, we have

$$
\operatorname{dim}_{\mathrm{VN}} \mathfrak{E}_{\lambda} \leq b+\operatorname{dim}_{\mathrm{VN}} \mathfrak{E}^{\prime} .
$$

We show $\mathfrak{E}^{\prime}$ is the trivial subspace by induction on the label $j$, showing that from low to high, any $f \in \mathfrak{E}^{\prime}$ vanishes on vertices with label $j$. Remember vertices $v$ can only be prodigy or bad.

Recall that we have finitely many labels. Let $j_{0}$ be the smallest label and let $v$ be of label $j_{0}$. If $v$ is a bad vertex, then $f(v)=0$ since $f \in \mathfrak{B}$. Note that $v$ cannot be a prodigy. Hence $f(v)=0$ on vertices of smallest label.

Now assume $f \in \mathfrak{E}^{\prime}$ vanishes on all vertices with label strictly below $j$. Since $f \in \mathfrak{B}$, we know $f$ vanishes on bad vertices. If $v$ is a prodigy vertex of label $j$, then $v$ has a neighbour $w$ such that $f$ vanishes on $w$ and all neighbours of $w$, except perhaps $v$. But (4.1) gives $\frac{f(v)}{S_{\lambda}\left(L_{v w}\right)}=W_{\lambda}(w) f(w)-\sum_{u \sim w, u \neq v} \frac{f(u)}{S_{\lambda}\left(L_{u v}\right)}$, so if the RHS is zero, then $f(v)=0$.

Recalling that $b \leq 1 / k$, we have showed that $\operatorname{dim} \mathfrak{E}_{\lambda} \leq 1 / k$. As $k$ is arbitrary, we get $\operatorname{dim} \mathfrak{E}_{\lambda}=0$. It follows that $\mathfrak{E}_{\lambda}=\{0\}$. Indeed, we have $P_{\mathfrak{E}_{\lambda}}(v, v)=0$ for all $v$, so tr $P_{\mathfrak{E}_{\lambda}}=0$, so $\left\|P_{\mathfrak{E}_{\lambda}}\right\|_{o p} \leq\left\|P_{\mathfrak{E}_{\lambda}}\right\|_{1}=0$, implying $\mathfrak{E}_{\lambda}=\{0\}$. It follows that there is no $\ell^{2}$ function on $\mathbb{T}$ such that $\mathscr{A}_{\lambda} \psi=W_{\lambda} \psi$. By [15], it follows that there is no $L^{2}$ function on $\mathbf{T}$ such that $H_{\mathbf{T}} \varphi=\lambda \varphi$. In other words, $\lambda$ is not an eigenvalue of $H_{\mathbf{T}}$ (contradiction).

4.3. More examples. Let $\mathbf{T}=\widetilde{\mathbf{G}}$. We now show the spectral bottom $a_{0}=\inf \sigma\left(H_{\mathbf{T}}\right)$ is strictly below the smallest Dirichlet value. By virtue of Proposition 4.1, if $G$ is Hamiltonian, this implies $a_{0}$ is not an eigenvalue. In particular, $a_{0}$ is not an isolated spectral value, so in view of Theorem 1.2, there is some pure AC spectrum near $a_{0}$.

Recall that if $Q_{j}$ are the quadratic forms associated to operators $H_{j}$, then $H_{1} \geq H_{2}$ if $D\left(Q_{1}\right) \subseteq D\left(Q_{2}\right)$ and $Q_{1}(f, f) \geq Q_{2}(f, f)$ for $f \in D\left(Q_{1}\right)$. If $H_{1} \geq H_{2}$ then $\inf \sigma\left(H_{1}\right) \geq \inf \sigma\left(H_{2}\right)$.

In $\mathbf{T}$ there are finitely many different kinds of edges (lengths and potentials). To each oriented edge $b$, we associate the smallest Dirichlet: the smallest $\mathscr{E}$ such that $S_{\mathscr{E}}\left(L_{b}\right)=0$. Denote the least of those values by $\mathscr{E}_{\mathrm{D}}$ and let $(v, w)$ be the edge on which it is attained (choose one of them if there is more than one edge with the same lowest Dirichlet value).

Consider the quantum star graph around $v$, with the usual $\delta$-condition at $v$, and Dirichlet conditions at the extremities $w^{\prime} \sim v$. Denote this (compact) graph by $\star$ and let $E_{0}$ be its smallest eigenvalue. We claim that

$$
a_{0} \leq E_{0}<\mathscr{E} \mathrm{D}
$$

For the first inequality, let $H_{\star} f=E_{0} f$. Then $E_{0}=\frac{Q_{\star}(f, f)}{\|f\|^{2}}$, where $Q_{\star}$ is the quadratic form associated to $H_{\star}$. Let $\tilde{f}$ be the extension of $f$ by zero to $\mathscr{T}$. Then $\tilde{f} \in D\left(Q_{\mathbf{T}}\right)$, where $Q_{\mathbf{T}}$ corresponds to $H_{\mathbf{T}}$. So

$$
a_{0}=\inf _{g \neq 0} \frac{Q_{\mathbf{T}}(g, g)}{\|g\|^{2}} \leq \frac{Q_{\mathbf{T}}(\tilde{f}, \tilde{f})}{\|\tilde{f}\|^{2}}=\frac{Q_{\star}(f, f)}{\|f\|^{2}}=E_{0} .
$$


For the second inequality, note that if $f \in D\left(H_{\star}\right)$ and $H_{\star} f=E f$, then on the edge $b$,

$$
f\left(x_{b}\right)=A C_{E}\left(x_{b}\right)+B S_{E}\left(x_{b}\right) .
$$

Due to the Dirichlet conditions at extremities of the star, $f\left(L_{b}\right)=0$. If $E<\mathscr{E}_{\mathrm{D}}$ then $S_{E}\left(L_{b}\right) \neq 0$ and $B=-A C_{E}\left(L_{b}\right) / S_{E}\left(L_{b}\right)$. Evaluating at $x_{b}=0$, the centre of the star, reveals that $A=f(v)$. Thus,

$$
f\left(x_{b}\right)=f(v) \frac{C_{E}\left(x_{b}\right) S_{E}\left(L_{b}\right)-C_{E}\left(L_{b}\right) S_{E}\left(x_{b}\right)}{S_{E}\left(L_{b}\right)} .
$$

The condition at $v, \sum_{b \in \star} f^{\prime}\left(o_{b}\right)=\alpha_{v} f(v)$, leads to

$$
\sum_{w^{\prime} \sim v}-\frac{C_{E}\left(L_{v w^{\prime}}\right)}{S_{E}\left(L_{v w^{\prime}}\right)}=\alpha_{v}
$$

Denote the left hand side of (4.2) as a function of $E$ as $Z(E)$. A solution $Z(E)=\alpha_{v}$ will be an eigenvalue of the star graph.

Let us consider the behaviour of $Z(E)$ as $E$ increases to $\mathscr{E}_{\mathrm{D}}$. We first show that as $E$ approaches $\mathscr{E}$ D from below, $S_{E}\left(L_{v w}\right) \rightarrow 0$ from above. For this, note that:

- by [26, Theorem 6(a)], $S_{\mathscr{E}_{\mathrm{D}}}(x)$ has exactly two zeros on $\left[0, L_{v w}\right]$. These are thus $\left\{0, L_{v w}\right\}$. If $E<\mathscr{D}$, since $S_{E}(0)=0$ and $S_{E}^{\prime}(0)=1$, we know that $S_{E}$ is positive near 0 . If we show that its first zero on $(0, \infty)$ occurs after $L_{v w}$, this will imply that $S_{E}\left(L_{v w}\right)>0$, which is what we seek.

- We thus check that if $E<\mathscr{E}_{\mathrm{D}}$, then the first zero of $S_{E}$ on $(0, \infty)$ occurs after the first zero of $S_{\mathscr{E}_{\mathrm{D}}}$. For this, let $f(x)=S_{E}(x) S_{\mathscr{E}_{\mathrm{D}}}^{\prime}(x)-S_{E}^{\prime}(x) S_{\mathscr{E}_{\mathrm{D}}}(x)$. Then $f^{\prime}(x)=$ $S_{E}(x) S_{\mathscr{E}_{\mathrm{D}}}(x)\left(E-\mathscr{E}_{\mathrm{D}}\right)$, which is negative until the first zero of $S_{E}$ or $S_{\mathscr{E}_{\mathrm{D}}}$. Suppose for contradiction that the first zero of $S_{E}$ (call it $L_{E}$ ) is before the first zero of $S_{\mathscr{E}_{\mathrm{D}}}$. Then we get that $f(0)=0, f\left(L_{E}\right)>0$, but $f^{\prime}(x)<0$ on $\left(0, L_{E}\right)$, which is absurd. This proves the claim.

Next, $S_{\mathscr{E} \mathrm{D}}^{\prime}\left(L_{v w}\right)<0$ because $L_{v w}$ is the first zero of $S_{\mathscr{E}_{\mathrm{D}}}$ after $x=0$. By the Wronskian relation $C_{\mathscr{E}_{\mathrm{D}}}\left(L_{v w}\right) S_{\mathscr{E}_{\mathrm{D}}}^{\prime}\left(L_{v w}\right)=1$, so $C_{\mathscr{E}_{\mathrm{D}}}\left(L_{v w}\right)<0$ too. Therefore, since $\mathscr{E}_{\mathrm{D}}$ is the smallest Dirichlet eigenvalue, all the terms in $Z(E)$ are either finite or diverge to $+\infty$ as $E \nearrow \mathscr{E}_{\mathrm{D}}$, so that $Z(E)$ diverges to $+\infty$ as $E \nearrow \mathscr{E}_{\mathrm{D}}$.

On the other hand, from the proof of Proposition 3.1, we know that $\frac{C_{E}\left(L_{b}\right)}{S_{E}\left(L_{b}\right)} \rightarrow+\infty$ as $E \rightarrow-\infty$, so $Z(E) \rightarrow-\infty$ in the same limit.

Together we have

$$
Z(E) \rightarrow-\infty \text { as } E \rightarrow-\infty \quad \text { and } \quad Z(E) \rightarrow+\infty \text { as } E \nearrow \mathscr{E} \text {. }
$$

Since $Z$ is continuous, there is a solution to $Z(E)=\alpha_{v}$ strictly below $\mathscr{E}$, as claimed.

\section{AC Spectrum Under Perturbations}

We now aim to prove Theorem 1.3. For this, we adapt the approach of [20], see also [16] for some earlier ideas.

A very sketchy outline of the argument is as follows. Our results in the previous sections tell us that we have a good control over the unperturbed operator: it has pure AC 
spectrum in $\Sigma$, and all relevant spectral quantities such as the Green's functions and WT functions have a limit on $\Sigma$, which has a strictly positive imaginary part. Let $H_{v}^{z}$ be such a spectral quantity to be chosen later, where $z \in \mathbb{C}^{+}$and $v \in V(\mathbb{T})$. The aim is now to prove an $L^{p}$-continuity estimate in mean with respect to the disorder $\varepsilon$. More precisely, in some semi-metric $\gamma$ on $\mathbb{H}$, see (5.1), we aim to show that $\lim _{\varepsilon \downarrow 0} \sup _{z \in I+\mathrm{i}(0,1)} \mathbb{E}\left(\gamma\left(h_{v}^{z}, H_{v}^{z}\right)^{p}\right)=$ 0 , where $h_{v}^{z}$ is the analogous spectral quantity for the perturbed operator (equal to $H_{v}^{z}$ when $\varepsilon=0$ ). Such a uniform stability result directly implies almost-sure pure AC spectrum in the combinatorial case, by classical results. In our case we will have to work further (Sect. 5.3 and "Appendix A.2").

The important question now is which Herglotz function to choose for $H_{v}^{z}$. In the combinatorial case it is natural to take $\zeta^{z}(b)$, with $b=\left(v_{-}, v\right)$. We considered something close in Proposition 3.1, namely $S_{z}\left(L_{b}\right) \zeta^{z}(b)$. For the present continuity considerations, $H_{v}^{z}=\frac{R_{z}^{+}\left(o_{b}\right)}{\sqrt{z}}$ seems to behave better. Still, the function $\sqrt{z} S_{z}\left(L_{b}\right) \zeta^{z}(b)$ will also play an important role in fixing the disorder window later on ("Appendix B"). Of course it can be argued that all such quantities are related in Sect. 2, but one needs to be careful because the aim is roughly to prove strict contraction estimates on $\mathbb{E}\left(\gamma\left(h_{v}^{z}, H_{v}^{z}\right)^{p}\right)$ in terms of $\sum_{w \in \mathscr{N}_{v}^{+}} \mathbb{E}\left(\gamma\left(h_{w}^{z}, H_{w}^{z}\right)^{p}\right)$, so adding/multiplying terms to $h_{w}^{z}$ is not a very good operation, although there are partial answers (Lemma 5.1 and Lemma 5.2).

We have not discussed how the $L^{p}$-continuity actually proceeds; we outline the proof in Sect. 5.2 after giving some important expansion estimates in Sect. 5.1.

5.1. The two step expansion estimate. Consider the hyperbolic disc $\mathbb{D}=\{z \in \mathbb{C}:|z|<$ 1 ) equipped with the usual hyperbolic distance metric

$$
d\left(z, z^{\prime}\right)=\cosh ^{-1}\left(1+\delta\left(z, z^{\prime}\right)\right)
$$

where

$$
\delta\left(z, z^{\prime}\right)=2 \frac{\left|z-z^{\prime}\right|^{2}}{\left(1-|z|^{2}\right)\left(1-\left|z^{\prime}\right|^{2}\right)}, \quad|z|,\left|z^{\prime}\right|<1
$$

We will use the Möbius transformation $\mathscr{C}(z)=\frac{z-\mathrm{i}}{z+i}$ that sends the upper half plane model $\mathbb{H}$ isometrically to the disk model. Its inverse is $\mathscr{C}^{-1}(u)=\mathrm{i} \frac{1+u}{1-u}$. Note that if, for $g, h \in \mathbb{H}$, we set

$$
\gamma(g, h)=\frac{|g-h|^{2}}{\operatorname{Im} g \operatorname{Im} h}
$$

then

$$
\gamma(g, h)=2 \delta(\mathscr{C}(g), \mathscr{C}(h))
$$

In fact,

$$
\delta(\mathscr{C} g, \mathscr{C} h)=2 \frac{|(g-\mathrm{i})(h+\mathrm{i})-(g+\mathrm{i})(h-\mathrm{i})|^{2}}{\left(|g+\mathrm{i}|^{2}-|g-\mathrm{i}|^{2}\right)\left(|h+\mathrm{i}|^{2}-|h-\mathrm{i}|^{2}\right)}=\frac{1}{2} \gamma(g, h) .
$$

The following is a more adequate replacement of [20, Lemma 1] to our framework. 
Lemma 5.1. Let $K$ be a compact subset of the hyperbolic disc $\mathbb{D}$. Then there exists a continuous function $C_{K}: \mathbb{R}^{+} \longrightarrow \mathbb{R}^{+}$, such that $C_{K}(0)=0$, and

$$
\delta\left(\lambda_{1} z, \lambda_{2} z^{\prime}\right) \leq\left(\left|\lambda_{1}\right|^{2}+C_{K}\left(\left|\lambda_{1}-\lambda_{2}\right|\right)\right) \delta\left(z, z^{\prime}\right)+C_{K}\left(\left|\lambda_{1}-\lambda_{2}\right|\right)
$$

for all $z \in K$ and for all $z^{\prime} \in \mathbb{D}$, for all $\lambda_{i} \in \mathbb{C}$ such that $\left|\lambda_{i}\right| \leq 1$.

More explicitly, if $r_{K}=\max _{z \in K}|z|<1$, we can take $C_{K}(t)=\frac{8}{\left(1-r_{K}\right)^{2}} \cdot t$.

Proof. First assume $\lambda_{1} \neq 0$. Suppose $z \neq z^{\prime}$. We have

$$
\begin{aligned}
\delta\left(\lambda_{1} z, \lambda_{2} z^{\prime}\right) & =\left|\lambda_{1}\right|^{2} \frac{\left|z-\lambda_{2} \lambda_{1}^{-1} z^{\prime}\right|^{2}}{\left|z-z^{\prime}\right|^{2}} \cdot \frac{\left(1-|z|^{2}\right)\left(1-\left|z^{\prime}\right|^{2}\right)}{\left(1-\left|\lambda_{1} z\right|^{2}\right)\left(1-\left|\lambda_{2} z^{\prime}\right|^{2}\right)} \cdot \delta\left(z, z^{\prime}\right) \\
& \leq\left|\lambda_{1}\right|^{2} \frac{\left|z-\lambda_{2} \lambda_{1}^{-1} z^{\prime}\right|^{2}}{\left|z-z^{\prime}\right|^{2}} \cdot \delta\left(z, z^{\prime}\right) \\
& \leq\left|\lambda_{1}\right|^{2}\left(1+\left|1-\lambda_{2} \lambda_{1}^{-1}\right| \frac{\left|z^{\prime}\right|}{\left|z-z^{\prime}\right|}\right)^{2} \delta\left(z, z^{\prime}\right),
\end{aligned}
$$

where, to obtain the first inequality, we used that $\left|\lambda_{i}\right| \leq 1$. Let $\delta_{K}=1-r_{K}$. If $\left|z-z^{\prime}\right| \geq \frac{\delta_{K}}{2}$, we have

$$
\begin{aligned}
\delta\left(\lambda_{1} z, \lambda_{2} z^{\prime}\right) & \leq\left(\left|\lambda_{1}\right|+\left|\lambda_{1}-\lambda_{2}\right|\left[1+\frac{|z|}{\left|z-z^{\prime}\right|}\right]\right)^{2} \delta\left(z, z^{\prime}\right) \\
& \leq\left(\left|\lambda_{1}\right|+\left(1+2 r_{K} \delta_{K}^{-1}\right)\left|\lambda_{1}-\lambda_{2}\right|\right)^{2} \delta\left(z, z^{\prime}\right) .
\end{aligned}
$$

Now assume $\left|z-z^{\prime}\right|<\frac{\delta_{K}}{2}$. Then $\left|z^{\prime}\right|<\frac{\delta_{K}}{2}+r_{K}=\frac{1+r_{K}}{2}$, so

$$
\begin{aligned}
\delta\left(\lambda_{1} z, \lambda_{2} z^{\prime}\right) & \leq 2\left|\lambda_{1}\right|^{2} \frac{\left(\left|z-z^{\prime}\right|+\left|1-\lambda_{2} \lambda_{1}^{-1}\right| \cdot\left|z^{\prime}\right|\right)^{2}}{\left(1-|z|^{2}\right)\left(1-\left|z^{\prime}\right|^{2}\right)} \\
& =\left|\lambda_{1}\right|^{2} \delta\left(z, z^{\prime}\right)+2 \frac{2\left|z-z^{\prime}\right| \cdot\left|\lambda_{1}\right|\left|\lambda_{1}-\lambda_{2}\right| \cdot\left|z^{\prime}\right|+\left|\lambda_{1}-\lambda_{2}\right|^{2} \cdot\left|z^{\prime}\right|^{2}}{\left(1-|z|^{2}\right)\left(1-\left|z^{\prime}\right|^{2}\right)} \\
& \leq\left|\lambda_{1}\right|^{2} \delta\left(z, z^{\prime}\right)+2 \frac{\frac{\delta_{K}\left(1+r_{K}\right)}{2} \cdot\left|\lambda_{1}-\lambda_{2}\right|+\left(\frac{1+r_{K}}{2}\right)^{2}\left|\lambda_{1}-\lambda_{2}\right|^{2}}{\left(1-r_{K}^{2}\right)\left(1-\left(\frac{1+r_{K}}{2}\right)^{2}\right)}
\end{aligned}
$$

The first assertion follows. For the explicit formula, we check the relevant terms in (5.3), (5.4) are bounded by $4 \frac{1+r_{K}}{\left(1-r_{K}\right)^{2}}\left|\lambda_{1}-\lambda_{2}\right|$. As $r_{K}<1$, this will complete the proof.

Note that $\left|\lambda_{1}-\lambda_{2}\right| \leq 2$, so

$$
\begin{aligned}
& 2\left(1+2 r_{K} \delta_{K}^{-1}\right)\left|\lambda_{1}\right|\left|\lambda_{1}-\lambda_{2}\right|+\left(1+2 r_{K} \delta_{K}^{-1}\right)^{2}\left|\lambda_{1}-\lambda_{2}\right|^{2} \\
& \quad \leq 2\left\{1+\frac{2 r_{K}}{1-r_{K}}+\left(1+\frac{2 r_{K}}{1-r_{K}}\right)^{2}\right\}\left|\lambda_{1}-\lambda_{2}\right|=4 \frac{1+r_{K}}{\left(1-r_{K}\right)^{2}}\left|\lambda_{1}-\lambda_{2}\right| .
\end{aligned}
$$

For the other term, using $\frac{1}{1-x^{2}} \leq \frac{1}{1-x}$ for $x \in[0,1]$, we have 


$$
\begin{aligned}
2 \frac{\frac{\delta_{K}\left(1+r_{K}\right)}{2} \cdot\left|\lambda_{1}-\lambda_{2}\right|+\left(\frac{1+r_{K}}{2}\right)^{2}\left|\lambda_{1}-\lambda_{2}\right|^{2}}{\left(1-r_{K}^{2}\right)\left(1-\left(\frac{1+r_{K}}{2}\right)^{2}\right)} & \leq \frac{1-r_{K}^{2}+\left(1+r_{K}\right)^{2}}{\left(1-r_{K}\right)\left(1-\left(\frac{1+r_{K}}{2}\right)\right)} \cdot\left|\lambda_{1}-\lambda_{2}\right| \\
& =4 \frac{1+r_{K}}{\left(1-r_{K}\right)^{2}}\left|\lambda_{1}-\lambda_{2}\right| .
\end{aligned}
$$

Finally, if $\lambda_{1}=0, \delta\left(\lambda_{1} z, \lambda_{2} z^{\prime}\right)=2 \frac{\left|\lambda_{2} z^{\prime}\right|^{2}}{1-\left|\lambda_{2} z^{\prime}\right|^{2}} \leq \frac{2\left|\lambda_{2}\right|}{1-\left|z^{\prime}\right|^{2}}$. If $\left|z-z^{\prime}\right| \geq \frac{\delta_{K}}{2}$ then $1 \leq \frac{4\left|z-z^{\prime}\right|^{2}}{\left(1-r_{K}\right)^{2}}$ and the claim follows. Otherwise $\left|z^{\prime}\right|<\frac{1+r_{K}}{2}$, so $\frac{1}{1-\left|z^{\prime}\right|^{2}} \leq \frac{2}{1-r_{K}}$, proving the claim.

We will also need [18, Lemma 2.16], which we recall below:

Lemma 5.2. For any $g, h, z \in \mathbb{H}$,

$$
\max \{\gamma(g, h+z), \gamma(g+z, h)\} \leq\left(1+c_{g}(z)\right) \gamma(g, h)+c_{g}(z),
$$

where $c_{g}(z)=\frac{4|z|}{\operatorname{Im} g}+\frac{4|z|^{2}}{(\operatorname{Im} g)^{2}}$.

We now consider $\mathbf{T}$ in the twisted view. If $\mathbb{T}$ is a tree with parameters $\left(\left\{\alpha_{v}^{0}\right\},\left\{L_{v}^{0}\right\}\right)$ before perturbation and $\left(\left\{\alpha_{v}^{\omega}\right\},\left\{L_{v}^{\omega}\right\}\right)$ after perturbation, and if $b=\left(v_{-}, v\right) \in B(\mathbf{T})=$ $B\left(\mathbf{T}_{o}^{+}\right)$, we set

$$
h_{v}^{z}=\frac{R_{z, \omega}^{+}\left(o_{b}\right)}{\sqrt{z}} \text { and } H_{v}^{z}=\frac{R_{z, 0}^{+}\left(o_{b}\right)}{\sqrt{z}},
$$

where $R_{z, \omega}^{+}\left(o_{b}\right)$ is the WT function of $\left(\mathbb{T},\left\{L_{v}^{\omega}\right\},\left\{\alpha_{v}^{\omega}\right\}\right)$ and $R_{z, 0}^{+}\left(o_{b}\right)$ the WT function of $\left(\mathbb{T},\left\{L_{v}^{0}\right\},\left\{\alpha_{v}^{0}\right\}\right)$. The notation may seem a bit confusing since the WT function is evaluated at $v_{-}$instead of $v$. However, this is in accordance with the notations of Sect. 1.1, where the oriented edges are indexed by their endpoint, and where we write $\phi_{v}$ instead of $\phi_{b}$ if $v=t_{b}$. We also define for $b=\left(v_{-}, v\right)$,

$$
\mathrm{g}_{v}^{z}=\frac{R_{z, \omega}^{+}\left(L_{b}^{\omega}\right)}{\sqrt{z}} \text { and } \Gamma_{v}^{z}=\frac{R_{z, 0}^{+}\left(L_{b}^{0}\right)}{\sqrt{z}} .
$$

Then the $\delta$-conditions (1.4) applied to $V_{z ; o}^{+}(x)$ give

$$
\sum_{v_{+} \in \mathscr{N}_{v}^{+}} h_{v_{+}}^{z}=\mathrm{g}_{v}^{z}+\frac{\alpha_{v}^{\omega}}{\sqrt{z}} \text { and } \sum_{v^{+} \in \mathscr{N}_{v}^{+}} H_{v_{+}}^{z}=\Gamma_{v}^{z}+\frac{\alpha_{v}^{0}}{\sqrt{z}}
$$

We shall assume the coupling constants $\alpha_{v} \geq 0$ and potentials $W \geq 0$. In this case we can ensure that $h_{v}^{z}$ and $H_{v}^{z}$ are Herglotz functions (Lemma 2.3), so their Cayley transform lies in $\mathbb{D}$. In this case, $\mathrm{g}_{v}^{z}$ and $\Gamma_{v}^{z}$ are also Herglotz by (5.5).

Remark 5.1. Assume there is no potential on the edges: $W_{v} \equiv 0$. Then the functions $h_{v}^{z}$ and $\mathrm{g}_{v}^{z}$ are also connected by the following relations: if $b=\left(v_{-}, v\right)$ and we expand $V_{z ; o}^{+}\left(x_{v}\right)$ in the basis $C_{z}\left(x_{v}\right)=\cos \sqrt{z} x_{v}$ and $S_{z}\left(x_{v}\right)=\frac{\sin \sqrt{z} x_{v}}{\sqrt{z}}$, we get

$$
R_{z}^{+}\left(t_{b}\right)=\frac{R_{z}^{+}\left(o_{b}\right) S_{z}^{\prime}\left(L_{b}\right)+C_{z}^{\prime}\left(L_{b}\right)}{R_{z}^{+}\left(o_{b}\right) S_{z}\left(L_{b}\right)+C_{z}\left(L_{b}\right)}=\frac{R_{z}^{+}\left(o_{b}\right) \cos \sqrt{z} L_{b}-\sqrt{z} \sin \sqrt{z} L_{b}}{R_{z}^{+}\left(o_{b}\right) \frac{\sin \sqrt{z} L_{b}}{\sqrt{z}}+\cos \sqrt{z} L_{b}},
$$


so $\mathrm{g}_{v}^{z}=\frac{h_{v}^{z} \cos \sqrt{z} L_{v}-\sin \sqrt{z} L_{v}}{h_{v}^{z} \sin \sqrt{z} L_{v}+\cos \sqrt{z} L_{v}}$. From this, we find

$$
\mathscr{C}\left(\mathrm{g}_{v}^{z}\right)=\frac{\mathrm{g}_{v}^{z}-\mathrm{i}}{\mathrm{g}_{v}^{z}+\mathrm{i}}=\mathscr{C}\left(h_{v}^{z}\right) \cdot \frac{\cos \sqrt{z} L_{v}-\mathrm{i} \sin \sqrt{z} L_{v}}{\cos \sqrt{z} L_{v}+\mathrm{i} \sin \sqrt{z} L_{v}}=\mathrm{e}^{-2 \mathrm{i} \sqrt{z} L_{v}} \mathscr{C}\left(h_{v}^{z}\right)
$$

as previously observed in [1]. We also remark that we can invert the Möbius identity defining $\mathrm{g}_{v}^{z}$ in terms of $h_{v}^{z}$ to get $h_{v}^{z}=\frac{\mathrm{g}_{v}^{z} \cos \sqrt{z} L_{v}+\sin \sqrt{z} L_{v}}{-\mathrm{g}_{v}^{z} \sin \sqrt{z} L_{v}+\cos \sqrt{z} L_{v}}$.

We finally define

$$
\gamma_{v}(h)=\gamma\left(h_{v}^{z}, H_{v}^{z}\right)
$$

The following plays the analog of [20, Lemma 2].

Lemma 5.3. Let $K$ be a compact subset of the hyperbolic disc, and assume that $z$ varies in a compact subset such that $H_{v}^{z} \in \mathbb{H}$ and $\mathscr{C}\left(\Gamma_{v}^{z}\right) \in K$ for all $v$. Then (see equations (5.6), (5.7) and (5.8) below for the definition of the quantities $q, \alpha$ and $Q$ )

$$
\begin{aligned}
& \gamma_{v}(h) \leq\left(1+C_{K}\left(\varepsilon^{\prime}\right)\right)\left(1+c_{H}(\varepsilon)\right) \\
& \quad \times \sum_{v^{\prime} \in \mathscr{N}_{v}^{+}} \frac{\operatorname{Im} H_{v^{\prime}}^{z} \cdot \gamma_{v^{\prime}}(h)}{\sum_{u \in \mathscr{N}_{v}^{+}} \operatorname{Im} H_{u}^{z}}\left(\sum_{w \in \mathscr{N}_{v}^{+}} q_{w}(h) Q_{v^{\prime}, w}(h) \cos \alpha_{v^{\prime}, w}(h)\right) \\
& \quad+2 C_{K}\left(\varepsilon^{\prime}\right)+\left(1+C_{K}\left(\varepsilon^{\prime}\right)\right) c_{H}(\varepsilon),
\end{aligned}
$$

if $\sup _{z, v}\left|\frac{\alpha_{v}^{\omega}-\alpha_{v}^{0}}{\sqrt{z}}\right| \leq \varepsilon$ and $\sup _{z, v}\left|\mathrm{e}^{2 \mathrm{i} \sqrt{z} L_{v}^{\omega}}-\mathrm{e}^{2 \mathrm{i} \sqrt{z} L_{v}^{0}}\right| \leq \varepsilon^{\prime}$, where $C_{K}(t)=\frac{8}{\left(1-r_{K}\right)^{2}} \cdot t$ and

$$
c_{H}(t)=\sup _{z, v} \frac{4 t}{\sum_{v_{+} \in \mathscr{N}_{v}^{+}} \operatorname{Im} H_{v_{+}}^{z}}\left(1+\frac{t}{\sum_{v_{+} \in \mathscr{N}_{v}^{+}} \operatorname{Im} H_{v_{+}}^{z}}\right) .
$$

We will apply this when $K=\bigcup_{v}\left\{\mathscr{C}\left(\Gamma_{v}^{z}\right): z \in I+\mathrm{i}[0,1]\right\}$, where $I$ is a compact interval on which $\Gamma_{v}^{\lambda+\mathrm{i} 0}$ exists and $\operatorname{Im} \Gamma_{v}^{\lambda+\mathrm{i} 0}>0$. Note that the union here is finite as the unperturbed model is of finite cone type. For the same reason, the supremum in $c_{H}$ is a maximum.

The quantities $q, Q, \alpha$ are defined by formulas similar to those in [20]: for $x, y \in$ $\mathscr{N}_{v}^{+}$,

$$
\begin{aligned}
q_{y}(h) & =\frac{\operatorname{Im} h_{y}}{\sum_{u \in \mathscr{N}_{v}^{+}} \operatorname{Im} h_{u}} \\
\cos \alpha_{x, y}(h) & =\cos \arg \left(h_{x}-H_{x}^{z}\right) \overline{\left(h_{y}-H_{y}^{z}\right)} \\
Q_{x, y}(h) & =\frac{\sqrt{\operatorname{Im} h_{x} \operatorname{Im} h_{y} \operatorname{Im} H_{x}^{z} \operatorname{Im} H_{y}^{z} \gamma_{x}(h) \gamma_{y}(h)}}{\frac{1}{2}\left(\operatorname{Im} h_{x} \operatorname{Im} H_{y}^{z} \gamma_{y}(h)+\operatorname{Im} h_{y} \operatorname{Im} H_{x}^{z} \gamma_{x}(h)\right)} .
\end{aligned}
$$

assuming $h_{x} \neq H_{x}^{z}$ and $h_{y} \neq H_{y}^{z}$, otherwise we let $Q_{x, y}(h)=\cos \alpha_{x, y}(h)=0$. 
Proof of Lemma 5.3. Using (5.2) and Remark 5.1, we have

$$
\gamma_{v}(h)=2 \delta\left(\mathscr{C}\left(H_{v}^{z}\right), \mathscr{C}\left(h_{v}^{z}\right)\right)=2 \delta\left(\mathrm{e}^{2 \mathrm{i} \sqrt{z} L_{v}^{0}} \mathscr{C}\left(\Gamma_{v}^{z}\right), \mathrm{e}^{2 \mathrm{i} \sqrt{z} L_{v}^{\omega}} \mathscr{C}\left(\mathrm{g}_{v}^{z}\right)\right) .
$$

By hypothesis, $\mathscr{C}\left(\Gamma_{v}^{z}\right)$ stays in $K$. Applying Lemma 5.1 and (5.2), we deduce that

$$
\gamma_{v}(h) \leq\left(1+C_{K}\left(\varepsilon^{\prime}\right)\right) \gamma\left(\Gamma_{v}^{z}, \mathrm{~g}_{v}^{z}\right)+2 C_{K}\left(\varepsilon^{\prime}\right) .
$$

Suppose $\alpha_{v}^{\omega} \geq \alpha_{v}^{0}$. Using (5.5), we need to estimate

$$
\gamma\left(\sum_{v_{+} \in \mathscr{N}_{v}^{+}} H_{v_{+}}^{z}-\frac{\alpha_{v}^{0}}{\sqrt{z}}, \sum_{v_{+} \in \mathscr{N}_{v}^{+}} h_{v_{+}}^{z}-\frac{\alpha_{v}^{\omega}}{\sqrt{z}}\right) \leq \gamma\left(\sum_{v_{+} \in \mathscr{N}_{v}^{+}} H_{v_{+}}^{z}, \sum_{v_{+} \in \mathscr{N}_{v}^{+}} h_{v_{+}}^{z}-\frac{\alpha_{v}^{\omega}-\alpha_{v}^{0}}{\sqrt{z}}\right),
$$

where the inequality holds by $\gamma\left(\xi+z, \xi^{\prime}+z\right) \leq \gamma\left(\xi\right.$, $\left.\xi^{\prime}\right)$ if $\operatorname{Im} z \geq 0$, as easily checked. Since we are assuming $\alpha_{v}^{\omega} \geq \alpha_{v}^{0} \geq 0$, we have $-\frac{\alpha_{v}^{\omega}-\alpha_{v}^{0}}{\sqrt{z}} \in \mathbb{H}$. Using Lemma 5.2, we may drop the term $\frac{\alpha_{v}^{\omega}-\alpha_{v}^{0}}{\sqrt{z}}$. If $\alpha_{v}^{0} \geq \alpha_{v}^{\omega}$, we simply replace (5.10) by

$\gamma\left(\sum_{v_{+} \in \mathscr{N}_{v}^{+}} H_{v_{+}}^{z}-\frac{\alpha_{v}^{0}}{\sqrt{z}}, \sum_{v_{+} \in \mathscr{N}_{v}^{+}} h_{v_{+}}^{z}-\frac{\alpha_{v}^{\omega}}{\sqrt{z}}\right) \leq \gamma\left(\sum_{v_{+} \in \mathscr{N}_{v}^{+}} H_{v_{+}}^{z}-\frac{\alpha_{v}^{0}-\alpha_{v}^{\omega}}{\sqrt{z}}, \sum_{v_{+} \in \mathscr{N}_{v}^{+}} h_{v_{+}}^{z}\right)$ and remove $\frac{\alpha_{v}^{0}-\alpha_{v}^{\omega}}{\sqrt{z}}$ using Lemma 5.2. Finally, as calculated in [20, Lemma 2],

$$
\left|\sum_{v_{+} \in \mathscr{N}_{v}^{+}} H_{v_{+}}^{z}-\sum_{v_{+} \in \mathscr{N}_{v}^{+}} h_{v_{+}}^{z}\right|^{2}=\sum_{v^{\prime} \in \mathscr{N}_{v}^{+}} \operatorname{Im} H_{v^{\prime}}^{z} \cdot \gamma_{v^{\prime}}(h)\left(\sum_{w \in \mathscr{N}_{v}^{+}} \operatorname{Im} h_{w}^{z} Q_{v^{\prime}, w}(h) \cos \alpha_{v^{\prime}, w}(h)\right) .
$$

Dividing by $\left(\operatorname{Im} \sum_{v_{+}} H_{v_{+}}\right)\left(\operatorname{Im} \sum_{v_{+}} h_{v_{+}}\right)$completes the proof.

Remark 5.2. Note that $\sum_{v_{+}} q_{v_{+}}=1$. On the other hand, $Q_{v^{\prime}, w}$ is a quotient of a geometric and arithmetic mean, so $0 \leq Q_{v^{\prime}, w} \leq 1$. Since $-1 \leq \cos \alpha_{v^{\prime}, w} \leq 1$, this shows that $-1 \leq \sum_{w \in \mathscr{N}_{v}} q_{w} Q_{v^{\prime}, w} \cos \alpha_{v^{\prime}, w} \leq 1$.

We now deduce a "two-step expansion". We will assume our tree satisfies (C2). In other words, for each vertex $v$, there is a vertex $v^{\prime} \in \mathscr{N}_{v}^{+}$such that every label found in $\mathscr{N}_{v}^{+}$can also be found in $\mathscr{N}_{v^{\prime}}^{+}$. We then say that $v^{\prime}$ is chosen w.r.t. (C2).

From now on, we denote

$$
S_{v}=\mathscr{N}_{v}^{+}
$$

If $*=t_{b_{o}}$ and $*^{\prime}$ is the vertex chosen w.r.t. (C2) corresponding to $*$, we let

$$
S_{*, *^{\prime}}=\left(\mathscr{N}_{*}^{+} \backslash\left\{*^{\prime}\right\}\right) \cup \mathscr{N}_{*^{\prime}}^{+} .
$$

Given $x \in S_{*}$, let

$$
c_{x}(h)=\sum_{y \in S_{*}} q_{y}(h) Q_{x, y}(h) \cos \alpha_{x, y}(h)
$$


and for $x \in S_{*^{\prime}}$, let

$$
\begin{aligned}
c_{x}(h) & =\left(\sum_{y \in S_{*}} q_{y}(h) Q_{*^{\prime}, y}(h) \cos \alpha_{*^{\prime}, y}(h)\right)\left(\sum_{y \in S_{*^{\prime}}} q_{y}(h) Q_{x, y}(h) \cos \alpha_{x, y}(h)\right) \\
& =c_{*^{\prime}}(h) \sum_{y \in S_{*^{\prime}}} q_{y}(h) Q_{x, y}(h) \cos \alpha_{x, y}(h) .
\end{aligned}
$$

It follows from Remark 5.2 that $c_{x}(h) \in[-1,1]$. We next define for $x \in S_{*}$,

$$
p_{x}=\frac{\operatorname{Im} H_{x}^{z}}{\sum_{y \in S_{*}} \operatorname{Im} H_{y}^{z}},
$$

and for $x \in S_{*^{\prime}}$,

$$
p_{x}=\frac{\operatorname{Im} H_{*^{\prime}}^{z} \operatorname{Im} H_{x}^{z}}{\left(\sum_{y \in S_{*}} \operatorname{Im} H_{y}^{z}\right)\left(\sum_{u \in S_{*^{\prime}}} \operatorname{Im} H_{u}^{z}\right)}=p_{*^{\prime}} \cdot \frac{\operatorname{Im} H_{x}^{z}}{\sum_{u \in S_{*^{\prime}}} \operatorname{Im} H_{u}^{z}} .
$$

Then $\sum_{x \in S_{*, *^{\prime}}} p_{x}=1$. Note that $c_{x}(h)$ is a quantity that depends on the random parameters of the perturbed graph, whereas $p_{x}$ is non-random.

Recall definition (1.9) of the set $\Sigma$. Using (5.5), we also have $\operatorname{Im} \Gamma_{v}^{\lambda+\mathrm{i} 0}>0$ on $\Sigma$.

Proposition 5.1. Let $I \subset \Sigma$ be a compact interval. There exists a continuous function $C_{I, H}:[0, \infty)^{2} \rightarrow[0, \infty)$ with $C_{I, H}(0,0)=0$ such that if $\sup _{z \in I+\mathrm{i}[0,1], v}\left|\frac{\alpha_{v}^{\omega}-\alpha_{v}^{0}}{\sqrt{z}}\right| \leq \varepsilon$ and $\sup _{z \in I+\mathrm{i}[0,1], v}\left|\mathrm{e}^{2 \mathrm{i} \sqrt{z} L_{v}^{\omega}}-\mathrm{e}^{2 \mathrm{i} \sqrt{z} L_{v}^{0}}\right| \leq \varepsilon^{\prime}$, then

$$
\gamma_{*}(h) \leq\left(1+C_{I, H}\left(\varepsilon, \varepsilon^{\prime}\right)\right) \sum_{x \in S_{*, *^{\prime}}} p_{x} c_{x}(h) \gamma_{x}(h)+C_{I, H}\left(\varepsilon, \varepsilon^{\prime}\right) .
$$

Proof. Let $K=\bigcup_{e}\left\{\mathscr{C}\left(\Gamma_{e}^{z}\right): z \in I+\mathrm{i}[0,1]\right\}$. We apply Lemma 5.3 to $v=*$, then to $v=*^{\prime}$. If $c_{I, H}\left(\varepsilon, \varepsilon^{\prime}\right)=2 C_{K}\left(\varepsilon^{\prime}\right)+c_{H}(\varepsilon)+C_{K}\left(\varepsilon^{\prime}\right) c_{H}(\varepsilon)$, the statement follows by taking $C_{I, H}\left(\varepsilon, \varepsilon^{\prime}\right)=2 c_{I, H}\left(\varepsilon, \varepsilon^{\prime}\right)+c_{I, H}\left(\varepsilon, \varepsilon^{\prime}\right)^{2}$.

To use this result under assumption $(\mathbf{P 0})$, note that

$$
\begin{aligned}
\left|\mathrm{e}^{2 \mathrm{i} \sqrt{z} L_{v}^{\omega}}-\mathrm{e}^{2 \mathrm{i} \sqrt{z} L_{v}^{0}}\right| & =\left|\mathrm{e}^{\mathrm{i} \sqrt{z}\left(L_{v}^{\omega}+L_{v}^{0}\right)}\right|\left|\mathrm{e}^{\mathrm{i} \sqrt{z}\left(L_{v}^{\omega}-L_{v}^{0}\right)}-\mathrm{e}^{\mathrm{i} \sqrt{z}\left(L_{v}^{0}-L_{v}^{\omega}\right)}\right| \\
& \leq 2 \sin \sqrt{z}\left(L_{v}^{\omega}-L_{v}^{0}\right) \\
& \leq 2 c_{I} \varepsilon .
\end{aligned}
$$

Thus, $\left|\mathrm{e}^{2 \mathrm{i} \sqrt{z} L_{v}^{\omega}}-\mathrm{e}^{2 \mathrm{i} \sqrt{z} L_{v}^{0}}\right| \leq \varepsilon^{\prime}$, with $\varepsilon^{\prime}=2 c_{I} \varepsilon$.

5.2. $L^{p}$ continuity of the WT function. The aim of this subsection is to establish the following uniform continuity result in $L^{p}$-norm:

Theorem 5.1. Let $\mathbb{T}$ satisfy (C0), (C1), (C2) and ( $\alpha, L)$ satisfy (P0), (P1) and (P2). For all compact $I \subset \Sigma, I \cap \mathscr{D}=\emptyset$, and $p>1$, there is $\varepsilon_{0}(I, p)>0, \eta_{0}\left(I, \varepsilon_{D}\right)>0$ and $C_{p}:\left[0, \varepsilon_{0}\right) \rightarrow[0, \infty)$ with $\lim _{\varepsilon \rightarrow 0} C_{p}(\varepsilon)=0$ such that for any $v \in \mathbb{T}, \varepsilon \leq \varepsilon_{0}$,

$$
\sup _{z \in I+\mathrm{i}\left(0, \eta_{0}\right]} \mathbb{E}\left(\gamma\left(h_{v}^{z}, H_{v}^{z}\right)^{p}\right) \leq C_{p}(\varepsilon), \sup _{z \in I+\mathrm{i}\left(0, \eta_{0}\right]} \mathbb{E}\left(\left|h_{v}^{z}-H_{v}^{z}\right|^{p}\right) \leq C_{p}(\varepsilon) .
$$


Given the key results of Sect. 5.1, much of the proof of Theorem 5.1 goes as in the combinatorial case [20], so we only outline the main ideas. The latter part of this proof becomes nevertheless more technical in the quantum setting, due to the more complicated relations among the Green's functions [see (5.23)], so we give the necessary modifications in "Appendix B".

Recall that $\mathbf{T}=\mathbf{T}_{o}^{+}$is defined by a cone matrix $M$ on a set of labels $\mathfrak{A}$ satisfying $(\mathbf{C} \mathbf{0})$, (C1) and (C2). We previously denoted $*=t_{b_{o}}$. More generally, given $\left(v_{-}, v\right) \in B\left(\mathbf{T}_{o}^{+}\right)$ with $\ell(v)=j$, if we consider the subtree $\mathbb{T}_{\left(v_{-}, v\right)}^{+}$, we shall denote $v=*_{j}$. The set $S_{*_{j}, *_{j}^{\prime}}$ is then constructed analogously, and all results of Sect. 5.1 apply without change (this works in particular for $*_{j}=o_{b_{o}}$ ).

Let us now discuss the proof of Theorem 5.1 in several steps:

Step 1: The Euclidean bound follows from the hyperbolic one.

In fact, if the $\gamma$-bound is proved, then using the Cauchy-Schwarz inequality,

$$
\mathbb{E}\left(\left|h_{v}^{z}-H_{v}^{z}\right|^{p}\right)^{2} \leq \mathbb{E}\left(\gamma\left(h_{v}^{z}, H_{v}^{z}\right)^{p}\right) \mathbb{E}\left(\left(\operatorname{Im} h_{v}^{z} \operatorname{Im} H_{v}^{z}\right)^{p}\right) \leq C(\varepsilon) \mathbb{E}\left(\left|h_{v}^{z}\right|^{p}\right)\left|H_{v}^{z}\right|^{p} .
$$

To bound the moment $\mathbb{E}\left(\left|h_{v}^{z}\right|^{p}\right)$, one uses the simple inequality

$$
|\xi| \leq 4 \gamma(\xi, \zeta) \operatorname{Im} \zeta+2|\zeta|, \quad \xi, \zeta \in \mathbb{H}
$$

applied to $\xi=h_{v}^{z}$ and $\zeta=H_{v}^{z}$.

Step 2: To prove the $\gamma$-bound, it suffices to show that for each $j \in \mathfrak{A}$,

$$
\mathbb{E}\left(\gamma\left(h_{*_{j}}^{z}, H_{*_{j}}^{z}\right)^{p}\right) \leq\left(1+c_{1}(\varepsilon)\right)\left(1-\delta_{0}\right) \sum_{k \in \mathfrak{A}} P_{j, k} \mathbb{E}\left(\gamma_{*_{k}}\left(h^{z}\right)^{p}\right)+C(\varepsilon),
$$

as long as $P=\left(P_{j, k}\right)$ forms a nonnegative irreducible matrix (and $c_{1}(\varepsilon), C(\varepsilon) \rightarrow 0$ as $\varepsilon \rightarrow 0$ ). Indeed, the Perron-Frobenius theorem then provides a positive eigenvector $u \in \mathbb{R}^{\mathfrak{A}}$ such that $P^{\top} u=u$. If we consider the vector $\mathbb{E}_{p} \gamma:=\left(\mathbb{E}\left(\gamma\left[h_{*_{j}}^{z}, H_{*_{j}}^{z}\right]^{p}\right)\right)_{j \in \mathfrak{A}}$, then (5.18) implies that

$$
\left\langle u, \mathbb{E}_{p} \gamma\right\rangle_{\mathbb{C}^{\mathfrak{A}}} \leq(1-\delta)\left\langle u, P \mathbb{E}_{p} \gamma\right\rangle_{\mathbb{C}^{\mathfrak{A}}}+C(\varepsilon)=(1-\delta)\left\langle u, \mathbb{E}_{p} \gamma\right\rangle_{\mathbb{C}^{\mathfrak{A}}}+C(\varepsilon),
$$

so $\left\langle u, \mathbb{E}_{p} \gamma\right\rangle_{\mathbb{C}^{\mathfrak{A}}} \leq \frac{C(\varepsilon)}{\delta}$, and the $\gamma$-bound easily follows.

Step 3: To prove (5.18), we apply the two-step expansion (Proposition 5.1) to get

$$
\begin{aligned}
\mathbb{E}\left(\gamma\left(h_{*_{j}}^{z}, H_{*_{j}}^{z}\right)^{p}\right) \leq & \left(1+C_{I, H}\right)^{2 p-1} \mathbb{E}\left(\left(\sum_{x \in S_{*_{j}, *_{j}^{\prime}}} p_{x} c_{x}\left(h^{z}\right) \gamma_{x}\left(h^{z}\right)\right)^{p}\right) \\
& +\left(1+C_{I, H}\right)^{p-1} C_{I, H}
\end{aligned}
$$

The idea now is that for $p \geq 1$,

$$
\left(\sum_{x \in S_{*_{j}, *_{j}^{\prime}}} p_{x} c_{x}(h) \gamma_{x}(h)\right)^{p} \leq \sum_{x \in S_{*_{j}, *_{j}^{\prime}}} p_{x} \gamma_{x}(h)^{p} \leq \max _{x \in S_{*_{j}, *_{j}^{\prime}}} \gamma\left(H_{x}^{z}, h_{x}^{z}\right)^{p}
$$


as follows from Jensen's inequality and the facts $\sum p_{x}=1$ and $\left|c_{x}(h)\right| \leq 1$. However the first inequality is generally strict for $p>1$, and this is what provides the $\left(1-\delta_{0}\right)$ in (5.18) if we choose $P=P(z)$ to be the matrix

$$
P_{j, k}:=\sum_{\substack{x \in S_{*_{j}, *_{j}^{\prime}}, \ell(x)=k}} p_{x}
$$

for $j, k \in \mathfrak{A}$, which satisfies the requirements of Step 2 .

To derive the strict contraction for (5.20) more precisely, the authors in [20] introduce an additional averaging over the permutations of $S_{*_{j}, *_{j}{ }_{j}}$ preserving the labels. Let

$$
\Pi_{j}:=\left\{\pi: S_{*_{j}, *_{j}^{\prime}} \rightarrow S_{*_{j}, *_{j}^{\prime}} \mid \pi \text { is bijective and } \ell(\pi(x))=\ell(x) \text { for all } x \in S_{*_{j}, *_{j}^{\prime}}\right\} .
$$

Fix $*=*_{j}$. Recall we denote functions $\phi_{b}$ on $\left[0, L_{b}\right]$ by $\phi_{v}$ if $b=\left(v_{-}, v\right)$. If $v \in S_{*, *^{\prime}}$ and $\pi \in \Pi$, then $\phi_{\pi v}:=\phi_{(*, \pi v)}$ if $\pi v \in S_{*}$, and $\phi_{\pi v}:=\phi_{\left(*^{\prime}, \pi v\right)}$ if $\pi v \in S_{*^{\prime}}$.

Denote $H^{z}=\left(H_{x}^{z}\right)_{x \in S_{*, *^{\prime}}} \in \mathbb{C}^{S_{*, *^{\prime}}}$. Given $g \in \mathbb{H}^{S_{*, *^{\prime}}}$ and $\pi \in \Pi$, we denote $g \circ \pi=\left(g_{\pi(x)}\right)_{x \in S_{*, *^{\prime}}}$. By (2.16) and the symmetry of the tree, we get for the unperturbed WT function

$$
H^{z}=H^{z} \circ \pi
$$

for all $\pi \in \Pi$. By (P1), (P2) and (2.16), $h_{x}^{z}$ and $h_{y}^{z}$ are independent and identically distributed for $x, y \in S_{*, *^{\prime}}$ that carry the same label. Hence,

$$
\mathbb{E}\left(f\left(h^{z}\right)\right)=\mathbb{E}\left(f\left(h^{z} \circ \pi\right)\right)
$$

for any integrable function $f$ and all $\pi \in \Pi$.

Recall from Remark 5.1 that

$$
h_{v}^{z}=\frac{\mathrm{g}_{v}^{z} \cos \sqrt{z} L_{v}+\sin \sqrt{z} L_{v}}{-\mathrm{g}_{v}^{z} \sin \sqrt{z} L_{v}+\cos \sqrt{z} L_{v}} .
$$

On the other hand, $\mathrm{g}_{v}^{z}=\sum_{v_{+}} h_{v_{+}}^{z}-\frac{\alpha_{v}}{\sqrt{z}}$. Given $g \in \mathbb{H}^{S_{*, *^{\prime}}}$, it is therefore natural to define $g_{*^{\prime}}=g_{*^{\prime}}(z, \alpha, L)$ by

$$
g_{*^{\prime}}=\frac{\phi_{*^{\prime}}(g) \cos \sqrt{z} L+\sin \sqrt{z} L}{-\phi_{*^{\prime}}(g) \sin \sqrt{z} L+\cos \sqrt{z} L}, \quad \phi_{*^{\prime}}(g)=-\frac{\alpha}{\sqrt{z}}+\sum_{x \in S_{*^{\prime}}} g_{x} .
$$

In particular, for $g=H^{z}=\left(H_{x}^{z}\right)$, we get $\left(H^{z}\right)_{*^{\prime}}\left(z, \alpha^{0}, L^{0}\right)=H_{*^{\prime}}^{z}$.

Introduce the notation $g_{x}^{(\pi)}=g_{\pi(x)}$ for $x \in S_{*, *^{\prime}}$. We also define

$$
g_{*^{\prime}}^{(\pi)}=\left(g^{\pi}\right)_{*^{\prime}}=\frac{\phi_{*^{\prime}}\left(g^{(\pi)}\right) \cos \sqrt{z} L+\sin \sqrt{z} L}{-\phi_{*^{\prime}}\left(g^{(\pi)}\right) \sin \sqrt{z} L+\cos \sqrt{z} L} .
$$

Note that $g_{*^{\prime}}^{(\pi)} \neq g_{\pi *^{\prime}}$, in fact $\pi *^{\prime}$ is not even defined. On the other hand we have $H_{*^{\prime}}^{(\pi)}\left(z, \alpha_{*^{\prime}}^{0}, L_{*^{\prime}}^{0}\right)=H_{*^{\prime}}$ by $(5.21)$.

Given $g \in \mathbb{H}^{S_{*, *^{\prime}}}, \pi \in \Pi$ and $g_{*^{\prime}}(z, \alpha, L)$ as in (5.23)-(5.24), we denote

$$
\gamma_{x}^{(\pi)}(g)=\gamma_{x}\left(g^{(\pi)}\right)=\left\{\begin{array}{l}
\gamma\left(g_{\pi(x)}, H_{x}^{z}\right): x \in S_{*, *^{\prime}}, \\
\gamma\left(g_{*^{\prime}}^{(\pi)}, H_{*^{\prime}}^{z}\right): x=*^{\prime} .
\end{array}\right.
$$


Then in view of (5.22), the mean in the RHS of (5.19) becomes

$$
\mathbb{E}\left(\left(\sum_{x \in S_{*_{j}, *_{j}^{\prime}}} p_{x} c_{x}(h) \gamma_{x}(h)\right)^{p}\right)=\frac{1}{\left|\Pi_{j}\right|} \mathbb{E}\left(\sum_{\pi \in \Pi_{j}}\left(\sum_{x \in S_{*_{j}, *_{j}^{\prime}}} p_{x} c_{x}^{(\pi)}(h) \gamma_{x}^{(\pi)}(h)\right)^{p}\right)
$$

To implement the strict inequality in (5.20), given $p \geq 1, z \in \mathbb{H}, \alpha, L>0$, one defines $\kappa_{*}^{(p)}:=\kappa_{*}^{(p)}(z, \alpha, L, \cdot): \mathbb{H}^{S_{*, *^{\prime}}} \rightarrow \mathbb{R}$, by

$$
\kappa_{*}^{(p)}(g):=\frac{\sum_{\pi \in \Pi}\left(\sum_{x \in S_{*, *^{\prime}}} p_{x} c_{x}^{(\pi)}(g) \gamma_{x}^{(\pi)}(g)\right)^{p}}{\sum_{\pi \in \Pi} \sum_{x \in S_{*, *^{\prime}}} p_{x} \gamma_{x}^{(\pi)}(g)^{p}} .
$$

Here, $\kappa_{*}^{(p)}$ depends on $\alpha, L$ via $c_{x}^{(\pi)}(g):=c_{x}\left(g^{(\pi)}\right)$. Indeed, ${ }^{7}$ recall that $c_{y}(g)$ involves $c_{*^{\prime}}(g)$ for $y \in S_{*^{\prime}}$, and $c_{*^{\prime}}(g)$ involves $g_{*^{\prime}}$, which in turns depends on $\alpha, L$.

The RHS of (5.25) now becomes

$$
\frac{1}{\left|\Pi_{j}\right|} \mathbb{E}\left(\kappa_{*_{j}}^{(p)}\left(z, \alpha_{*_{j}^{\prime}}^{\omega}, L_{*_{j}^{\prime}}^{\omega}, h\right) \sum_{\pi \in \Pi_{j}} \sum_{x \in S_{*_{j}, *_{j}^{\prime}}} p_{x} \gamma_{x}^{(\pi)}(h)^{p}\right)
$$

If all $\gamma_{x}^{(\pi)}(h) \approx 0$ then we are done, so the nontrivial work is when $h \notin B_{r}(H)$, where

$$
B_{r}(H):=\left\{g \in \mathbb{H}^{S_{*_{j}}, *_{j}^{\prime}}: \gamma\left(g_{x}, H_{x}^{z}\right) \leq r \forall x \in S_{*_{j}, *_{j}^{\prime}}\right\}
$$

This case is controlled by Proposition 5.2 below. To state it, recall that we assume $I$ is away from the Dirichlet spectrum in (1.10). This implies, for the $L$ in $\kappa_{*}^{(p)}$,

$$
\min _{z \in I+\mathrm{i}[0,1]}\left|\sin (\sqrt{z} L) \sin \left(\sqrt{z} L_{*^{\prime}}^{0}\right)\right| \geq \varepsilon_{D}>0 .
$$

Proposition 5.2. Let $I \subset \Sigma$ be compact and satisfy (1.10) and let $p>1$. For any $*=*_{j}$, there exist $\delta_{*}(I, p)>0, \varepsilon_{*}\left(I, \varepsilon_{D}\right)>0, \eta_{*}\left(I, \varepsilon_{D}\right)>0$ and $R_{*}:\left[0, \varepsilon_{*}\right] \rightarrow[0, \infty)$ with $\lim _{\varepsilon \rightarrow 0} R_{*}(\varepsilon)=0$ such that for all $\varepsilon \in\left[0, \varepsilon_{*}\right]$,

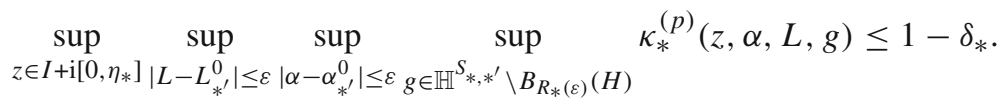

Proposition 5.2 is remarkable as it gives a uniform contraction estimate on the random variable. Using it in (5.27), we get the $\left(1-\delta_{0}\right)$ we were seeking in $(5.18)$ (take $\delta_{0}=$ $\left.\min _{j \in \mathfrak{A}} \delta_{*_{j}}\right)$, thus completing the proof of Theorem 5.1.

The proof of Proposition 5.2 is given in "Appendix B".

\footnotetext{
${ }^{7}$ Note that $c_{x}^{(\pi)}(g) \neq c_{\pi x}(g)$ in general. For example, if $x \in S_{*}$ and $\pi x \in S_{*^{\prime}}$, then $c_{x}^{(\pi)}(g)$ is defined by a single sum while $c_{\pi x}(g)$ is a product of two sums, as in (5.11)-(5.12).
} 
5.3. Proof of pure AC spectrum. We may finally conclude with the proof of Theorem 1.3, which we recall:

Theorem 5.2. Let $\mathbb{T}$ satisfy (C0), (C1), (C2). For all compact $I \subset \Sigma, I \cap \mathscr{D}=\emptyset$, and $p>1$, there is $\varepsilon_{0}(I, p)>0, \eta_{0}\left(I, \varepsilon_{D}\right)>0, C_{p}^{\prime}\left(I, \varepsilon_{D}\right)>0$ such that for any $v \in \mathbb{T}$,

$$
\sup _{z \in I+\mathrm{i}\left(0, \eta_{0}\right]} \mathbb{E}\left(\left|\operatorname{Im} R_{z}^{ \pm}(v)\right|^{-p}\right) \leq C_{p}^{\prime} \text { and } \sup _{z \in I+\mathrm{i}\left(0, \eta_{0}\right]} \mathbb{E}\left(\left|R_{z}^{ \pm}(v)\right|^{ \pm p}\right) \leq C_{p}^{\prime}
$$

for all $\varepsilon \in\left[0, \varepsilon_{0}\right)$ and all $\left(\alpha^{\omega}, L^{\omega}\right)$ satisfying $(\boldsymbol{P 0}),(\boldsymbol{P} 1)$ and $(\boldsymbol{P} 2)$.

In particular, $\mathscr{H}_{\varepsilon}^{\omega}$ has pure AC spectrum almost surely in $I$.

Proof. We showed after (5.16) that $\sup _{z \in I+\mathrm{i}\left(0, \eta_{0}\right]} \mathbb{E}\left(\left|h_{x}^{z}\right|^{p}\right)<\infty$. So for $b=\left(v_{-}, v\right)$,

$$
\mathbb{E}\left(\left|R_{z, \omega}^{+}(v)\right|^{p}\right)=|z|^{p / 2} \mathbb{E}\left(\left|h_{v}^{z}\right|^{p}\right) \leq C_{p}
$$

for any $z \in I+\mathrm{i}\left(0, \eta_{0}\right]$. On the other hand, observe that

$$
\gamma\left(\frac{-1}{\mathrm{i} \operatorname{Im} h_{v}^{z}}, \frac{-1}{\mathrm{i} \operatorname{Im} H_{v}^{z}}\right)=\gamma\left(\mathrm{i} \operatorname{Im} h_{v}^{z}, \mathrm{i} \operatorname{Im} H_{v}^{z}\right) \leq \gamma\left(h_{v}^{z}, H_{v}^{z}\right) .
$$

In particular, using (5.17) with $\xi=\mathrm{i} / \operatorname{Im} h_{v}^{z}$ and $\zeta=\mathrm{i} / \operatorname{Im} H_{v}^{z}$, we get

$$
\left|\frac{1}{\operatorname{Im} h_{v}^{z}}\right|^{p} \leq 2^{p-1}\left\{4^{p} \frac{\gamma\left(h_{v}^{z}, H_{v}^{z}\right)^{p}}{\left(\operatorname{Im} H_{v}^{z}\right)^{p}}+2^{p}\left|\frac{1}{\operatorname{Im} H_{v}^{z}}\right|^{p}\right\} .
$$

Hence,

$$
\mathbb{E}\left(\left|\operatorname{Im} h_{v}^{z}\right|^{-p}\right) \leq C_{p}^{\prime}
$$

for any $z \in I+\mathrm{i}\left(0, \eta_{0}\right]$, by (5.15). In particular,

$$
\mathbb{E}\left(\left|R_{z, \omega}^{+}(v)\right|^{-p}\right)=|z|^{-p / 2} \mathbb{E}\left(\left|h_{v}^{z}\right|^{-p}\right) \leq C_{p}^{\prime \prime} .
$$

On the other hand, for any $b, R_{z}^{-}\left(t_{b}\right)=R_{z}^{+}\left(o_{\widehat{b}}\right)$, and we know all results hold true for the tree $\mathbf{T}_{t_{b}}^{-}=\mathbf{T}_{\sigma_{\hat{b}}}^{+}$(recall we are assuming $(\mathbf{C 1})$ ). So we also have $\mathbb{E}\left(\left|R_{z, \omega}^{-}(v)\right|^{p}\right)<\infty$ for any $p \in \mathbb{Z}$.

Finally, for $\left(\operatorname{Im} R_{z}^{+}(v)\right)^{-p}$, denote $\sqrt{z}=a+\mathrm{i} b$. Then

$$
\operatorname{Im} h_{v}^{z}=\frac{a \operatorname{Im} R_{z}^{+}(v)-b \operatorname{Re} R_{z}^{+}(v)}{|z|}=: \frac{F_{z}}{|z|} .
$$

Hence,

$$
\begin{aligned}
\mathbb{E}\left(\frac{1}{\operatorname{Im} R_{z}^{+}(v)^{p}}\right)= & a^{p} \mathbb{E}\left(\frac{1}{\left|F_{z}+b \operatorname{Re} R_{z}^{+}(v)\right|^{p}}\right) \\
= & a^{p} \mathbb{E}\left(\frac{1}{\left|F_{z}+b \operatorname{Re} R_{z}^{+}(v)\right|^{p}} \mathbb{1}_{\left\{\left|F_{z}\right|>\left|2 b \operatorname{Re} R_{z}^{+}(v)\right|\right\}}(\omega)\right) \\
& +a^{p} \mathbb{E}\left(\frac{1}{\left|F_{z}+b \operatorname{Re} R_{z}^{+}(v)\right|^{p}} \mathbb{1}_{\left\{\left|F_{z}\right| \leq\left|2 b \operatorname{Re} R_{z}^{+}(v)\right|\right\}}(\omega)\right) .
\end{aligned}
$$

For the first expectation, we have $\left|F_{z}+b \operatorname{Re} R_{z}^{+}(v)\right| \geq\left|F_{z}\right|-b\left|\operatorname{Re} R_{z}^{+}(v)\right| \geq \frac{1}{2}\left|F_{z}\right|$. For the second expectation, we make use of the lower-bound for $\operatorname{Im} R_{z}^{+}(v) / \operatorname{Im} z$ proved in the 
"Appendix" (Lemma A.2) to conclude that there is some $C>0$ with $\left|F_{z}+b \operatorname{Re} R_{z}^{+}(v)\right|=$ $a \operatorname{Im} R_{z}^{+}(v) \geq a C \operatorname{Im} z$ for any $z, v, \omega$. Summarizing, we get

$$
\begin{aligned}
\mathbb{E}\left(\operatorname{Im} R_{z}^{+}(v)^{-p}\right) \leq & (2 a)^{p} \mathbb{E}\left(\left|F_{z}\right|^{-p}\right)+C^{-p}(\operatorname{Im} z)^{-p} \mathbb{P}\left(\left|F_{z}\right| \leq\left|2 b \operatorname{Re} R_{z}^{+}(v)\right|\right) \\
\leq & (2 a)^{p}|z|^{-p} \mathbb{E}\left(\left(\operatorname{Im} h_{v}^{z}\right)^{-p}\right) \\
& +C^{-p}|z|^{-p}(\operatorname{Im} z)^{-p}(2 b)^{p} \mathbb{E}\left(\left|\frac{\operatorname{Re} R_{z}^{+}(v)}{\operatorname{Im} h_{v}^{z}}\right|^{p}\right)
\end{aligned}
$$

using Markov's inequality. The right-hand side is bounded uniformly in $z \in I+\mathrm{i}\left(0, \eta_{0}\right]$, since $\frac{2 b}{\operatorname{Im} z}=\frac{2 \operatorname{Im} \sqrt{z}}{\operatorname{Im} z}=\frac{1}{\operatorname{Re} \sqrt{z}}$. This completes the proof of (5.29).

The consequence on pure AC spectrum follows from Theorem A.1.

5.4. Uniform inverse moments. We now aim to prove Theorem 1.4. The arguments of this section are inspired from their analogs $[2,4]$ in case of regular combinatorial graphs.

Note that (P0) implies that the distributions $v_{j}$ of $\alpha_{v}^{\omega}$ have compact support supp $v_{j} \subseteq$ $\left[\alpha_{\min }-\varepsilon, \alpha_{\max }+\varepsilon\right]$. In particular, all moments $M_{\varsigma}=\max _{v} \mathbb{E}\left[\left|\alpha_{v}^{\omega}\right|^{\varsigma}\right]<\infty$ exist.

As in Sect. 3, we denote $R_{z}^{+}(k):=R_{z}^{+}\left(o_{b}\right)$ if $\ell\left(t_{b}\right)=k$. Note that here $R_{z}^{+}\left(o_{b}\right)$ is random; even if $\ell\left(t_{b}\right)=\ell\left(t_{b^{\prime}}\right)$ this doesn't imply that $R_{z}^{+}\left(o_{b}\right)$ and $R_{z}^{+}\left(o_{b^{\prime}}\right)$ are equal. However their distribution are the same, which justifies the notation for the purposes of this section. We also let $Q=\max _{v \in \mathbb{T}} \operatorname{deg}(v)-1$ and $q=\min _{v \in \mathbb{T}} \operatorname{deg}(v)-1$.

Let $I \subset \Sigma$ be compact with $I \cap \mathscr{D}=\emptyset$. Fix $\eta_{0}\left(I, \varepsilon_{D}\right)$ as in Theorem 5.2. Given $\delta \in(0,1)$ and $\varepsilon>0$, we introduce

$$
\sigma_{a c}^{\varepsilon}(\delta)=\left\{\lambda \in \mathbb{R}: \mathbb{P}\left(\operatorname{Im} R_{\lambda+\mathrm{i} \eta}^{+}(j)>\delta\right)>\delta \quad \forall j \in \mathfrak{A}, \forall \eta \in\left(0, \eta_{0}\right)\right\}
$$

Lemma 5.4. There exist $\varepsilon_{0}, \delta>0$ such that $I \subseteq \sigma_{a c}^{\varepsilon}(\delta)$ for $\varepsilon \leq \varepsilon_{0}$.

Proof. By Theorem 5.2, fixing $p=2$ we find $\varepsilon_{0}(I), C_{2}$ such that $\mathbb{E}\left(\left|\operatorname{Im} R_{z}^{ \pm}(j)\right|^{-2}\right) \leq$ $C_{2}$ so by Jensen $\mathbb{E}\left(\operatorname{Im} R_{z}^{ \pm}(j)\right) \geq \mathbb{E}\left(\left|\operatorname{Im} R_{z}^{ \pm}(j)\right|^{-2}\right)^{-1 / 2} \geq C_{2}^{-1 / 2}>0$.

Suppose that $\mathbb{P}\left(\operatorname{Im} R_{\lambda+\mathrm{i} \eta}^{+}(j)>\delta\right) \leq \delta$ for some $\lambda \in I, j \in \mathfrak{A}$ and $\eta \in\left(0, \eta_{0}\right)$. Then

$$
\begin{aligned}
\mathbb{E}\left[\operatorname{Im} R_{\lambda+\mathrm{i} \eta}^{+}(j)\right]= & \mathbb{E}\left[\operatorname{Im} R_{\lambda+\mathrm{i} \eta}^{+}(j) 1_{\operatorname{Im} R_{\lambda+\mathrm{i} \eta}^{+}(j)>\delta}\right] \\
& +\mathbb{E}\left[\operatorname{Im} R_{\lambda+\mathrm{i} \eta}^{+}(j) 1_{\operatorname{Im} R_{\lambda+\mathrm{i} \eta}^{+}(j) \leq \delta}\right] \leq\left(C_{2} \delta\right)^{1 / 2}+\delta<C_{2}^{-1 / 2}
\end{aligned}
$$

if $\delta>0$ is small enough, yielding a contradiction. Here, we used Cauchy-Schwarz and the bound $\mathbb{E}\left[\left|\operatorname{Im} R_{\lambda+i \eta}^{+}(j)\right|^{2}\right] \leq C_{2}$. Hence, $I \subseteq \sigma_{a c}^{\varepsilon}(\delta)$ for some $\delta>0$ as claimed.

Given $z \in \mathbb{C}^{+}$and $j \in \mathfrak{A}$, let

$$
F_{z}^{j}(x)=\mathbb{P}\left(\operatorname{Im} R_{z}^{+}(j) \leq x\right), \quad F_{z}(x)=\max _{j \in \mathfrak{A}} F_{z}^{j}(x) \quad \text { and } \quad H_{z}^{j}(x)=\mathbb{P}\left(\left|\zeta_{j}^{z}\right| \leq x\right) .
$$


Lemma 5.5. Let $I \subset \mathbb{R}$ be compact, $I \cap \mathscr{D}=\emptyset, \varsigma \geq 3, \lambda \in I, \eta>0$ and $z=\lambda+\mathrm{i} \eta$. Then

$$
F_{z}(x) \leq F_{z}\left(x y^{-2}\right)^{q}+C_{\nu, Q, \varsigma}\left(y^{\beta} F_{z}\left(\frac{4 Q c_{2}}{c_{1}^{2}} y\right)^{q}+y^{\varsigma}\right)
$$

for all $x>0$ and $y \in\left(0, c_{I}\right]$, where $\beta$ is from $(\boldsymbol{P 3}), c_{I}=\frac{c_{1}}{4 Q c_{2} c_{3}}$ and $c_{1}, c_{2}, c_{3}>0$ are chosen as in (2.18).

Proof. By (2.17) we have

$$
\operatorname{Im} R_{z}^{+}\left(o_{b}\right) \geq\left|\zeta^{z}(b)\right|^{2} \sum_{b^{+} \in \mathscr{N}_{b}^{+}} \operatorname{Im} R_{z}^{+}\left(o_{b^{+}}\right) .
$$

So given $y>0$, if $\operatorname{Im} R_{z}^{+}\left(o_{b}\right) \leq x$, then either $\left|\zeta^{z}(b)\right| \leq y$, or $\operatorname{Im} R_{z}^{+}\left(o_{b^{+}}\right) \leq x y^{-2}$ for all $b^{+} \in \mathscr{N}_{b}^{+}$. Recalling the matrix $M=\left(M_{i, j}\right)$ we thus have by independence,

$$
F_{z}^{j}(x) \leq F_{z}^{1}\left(x y^{-2}\right)^{M_{j, 1}} \cdots F_{z}^{m}\left(x y^{-2}\right)^{M_{j, m}}+H_{z}^{j}(y) \leq F_{z}\left(x y^{-2}\right)^{q}+H_{z}^{j}(y) .
$$

Now, assuming $c_{1} \leq\left|S_{z}\left(L_{b}\right)\right| \leq c_{2}$ and $\left|C_{z}\left(L_{b}\right)\right| \leq \frac{c_{1}}{4 Q c_{2} y} \leq \frac{1}{4 y}$ we have by (2.6)

$$
\begin{aligned}
H_{z}^{j}(y) & =\mathbb{P}\left(\left|S_{z}\left(L_{b}\right)\left(\alpha_{t_{b}}+\frac{S_{z}^{\prime}\left(L_{b}\right)}{S_{z}\left(L_{b}\right)}-\sum_{b^{+} \in \mathscr{N}_{b}^{+}} R_{z}^{+}\left(o_{b^{+}}\right)\right)\right| \geq y^{-1}\right) \\
& \leq \mathbb{P}\left(\left|\alpha_{t_{b}} S_{z}\left(L_{b}\right)\right|+\left|C_{z}\left(L_{b}\right)\right|+\left|S_{z}\left(L_{b}\right)\right| \sum_{b^{+} \in \mathscr{N}_{b}^{+}}\left|R_{z}^{+}\left(o_{b^{+}}\right)\right| \geq y^{-1}\right) \\
& \leq \mathbb{P}\left(c_{2}\left|\alpha_{t_{b}}\right|+(4 y)^{-1}+c_{2} \sum_{b^{+} \in \mathscr{N}_{b}^{+}}\left|R_{z}^{+}\left(o_{b^{+}}\right)\right| \geq y^{-1}\right) \\
& \leq \mathbb{P}\left(\left|\alpha_{t_{b}}\right| \geq\left(4 c_{2} y\right)^{-1}\right)+\mathbb{P}\left(\sum_{b^{+} \in \mathscr{N}_{b}^{+}}\left|R_{z}^{+}\left(o_{b^{+}}\right)\right| \geq\left(2 c_{2} y\right)^{-1}\right) \\
& \leq\left(4 c_{2} y\right)^{\varsigma} M_{\varsigma}+\sum_{k=1}^{m} M_{j, k} \mathbb{P}\left(\left|R_{z}^{+}(k)\right| \geq\left(2 Q c_{2} y\right)^{-1}\right),
\end{aligned}
$$

where $M_{\varsigma}=\max _{v} \mathbb{E}\left[\left|\alpha_{v}^{\omega}\right|^{\varsigma}\right]<\infty$. But for $t=\frac{1}{2 Q c_{2} y}$,

$$
\begin{aligned}
\mathbb{P} & \left(\left|R_{z}^{+}\left(o_{b}\right)\right| \geq t\right) \\
& =\mathbb{P}\left(\frac{\left|\zeta^{z}(b)-C_{z}\left(L_{b}\right)\right|}{\left|S_{z}\left(L_{b}\right)\right|} \geq t\right) \leq \mathbb{P}\left(\frac{\left|\zeta^{z}(b)\right|+\left(c_{1} / 4 Q c_{2} y\right)}{c_{1}} \geq t\right) \\
& \leq \mathbb{P}\left(\left|\zeta^{z}(b)\right| \geq \frac{c_{1} t}{2}\right) \leq \mathbb{P}\left(\left|\zeta^{z}(b) S_{z}\left(L_{b}\right)\right| \geq \frac{c_{1}^{2} t}{2}\right) \\
& \leq \mathbb{P}\left(\left|\alpha_{t_{b}}+\operatorname{Re} \frac{S_{z}^{\prime}\left(L_{b}\right)}{S_{z}\left(L_{b}\right)}-\sum_{b^{+} \in \mathscr{N}_{b}^{+}} \operatorname{Re} R_{z}^{+}\left(o_{b^{+}}\right)\right| \leq \frac{2}{c_{1}^{2} t} \text { and } \sum_{b^{+} \in \mathscr{N}_{b}^{+}} \operatorname{Im} R_{z}^{+}\left(o_{b^{+}}\right) \leq \frac{2}{c_{1}^{2} t}\right) \\
& \leq \mathbb{P}\left(\alpha_{t_{b}} \text { lies in an interval of length } \frac{4}{c_{1}^{2} t} \text { and } \sum_{b^{+} \in \mathscr{N}_{b}^{+}} \operatorname{Im} R_{z}^{+}\left(o_{b^{+}}\right) \leq \frac{2}{c_{1}^{2} t}\right)
\end{aligned}
$$




$$
\leq C_{v}\left(\frac{4}{c_{1}^{2} t}\right)^{\beta} \mathbb{P}\left(\sum_{b^{+} \in \mathscr{N}_{b}^{+}} \operatorname{Im} R_{z}^{+}\left(o_{b^{+}}\right) \leq \frac{2}{c_{1}^{2} t}\right) \leq C_{v}\left(\frac{4}{c_{1}^{2} t}\right)^{\beta} F_{z}\left(\frac{4 Q c_{2} y}{c_{1}^{2}}\right)^{q}
$$

Here we used that $-\frac{S_{z}^{\prime}(L)}{S_{z}(L)}$ is Herglotz and that $\left\{R_{z}^{+}\left(o_{b^{+}}\right)\right\}$are independent of $\alpha_{t_{b}}$, as follows from (2.16), and bounded the probability by first conditioning over the random variables different from $\alpha_{t_{b}}^{\omega}$, so that the "interval" above is fixed.

Thus, if $c_{I}=\frac{c_{1}}{4 q c_{2} c_{3}}$, where $\left|C_{z}\left(L_{b}\right)\right| \leq c_{3}$, then for any $0<y \leq c_{I} \leq \frac{c_{1}}{4 q c_{2}\left|C_{z}\left(L_{b}\right)\right|}$,

$$
H_{z}(y) \leq\left(4 c_{2}\right)^{\varsigma} M_{\varsigma} \cdot y^{\varsigma}+C_{v}\left(\frac{8 Q c_{2}}{c_{1}^{2}}\right)^{\beta} Q \cdot y^{\beta} \cdot F_{z}\left(\frac{4 Q c_{2} y}{c_{1}^{2}}\right)^{q} .
$$

Theorem 5.3. Let $I \subset \sigma_{a c}^{\varepsilon}(\delta)$ be bounded, $\lambda \in I, \varsigma \geq 3, \eta \in\left(0, \eta_{0}\right)$ and $z=\lambda+\mathrm{i} \eta$. Then

$$
F_{z}(x) \leq C_{v, Q, \delta, \varsigma} x^{\beta \varsigma / 5}
$$

for all $x \in\left(0, x_{\delta}\right]$, for some $C_{v, Q, \delta}<\infty$ and $x_{\delta}=x(\delta, v, Q, \varsigma, I)>0$.

Proof. The theorem is proved by gradually improving on the decay. First, take $y=x^{1 / 4}$ in Lemma 5.5 to get for $x \in\left(0, c_{I}^{4}\right]$,

$$
F_{z}(x) \leq F_{z}\left(x^{1 / 2}\right)^{q}+C_{v, Q}\left(x^{\beta / 4} F_{z}\left(\frac{4 Q c_{2}}{c_{1}^{2}} x^{1 / 4}\right)^{q}+x^{\varsigma / 4}\right),
$$

so using the bounds $x^{\beta / 2}\left[F_{z}\left(\frac{4 Q c_{2}}{c_{1}^{2}} x^{1 / 2}\right)\right]^{q} \leq x^{\beta / 2}$ and $x^{\varsigma / 2} \leq x^{\beta / 2}$ for small $x$, we get

$$
F_{z}\left(x^{2}\right) \leq F_{z}(x)^{q}+C_{\nu, Q}^{\prime} x^{\beta / 2} .
$$

Since $\lambda \in \sigma_{a c}^{\varepsilon}(\delta)$, we have $F_{z}(x)=\max _{j} \mathbb{P}\left(\operatorname{Im} R_{z}^{+}(j) \leq \delta\right) \leq 1-\delta$ for any $x \in[0, \delta]$. Choose $x_{0} \leq \min \left(\delta, c_{I}^{4},\left(\frac{\delta}{4 C_{v, Q}^{\prime}}\right)^{8 / \beta}\right)$. For $\alpha$ small enough, we have $(1-\delta) \leq\left(1-\frac{\delta}{2}\right) x_{0}^{2 \alpha}$. So there is some $\alpha_{0} \in(0, \beta / 16]$ such that

$$
F_{z}\left(x_{0}\right) \leq\left(1-\frac{\delta}{2}\right) x_{0}^{2 \alpha_{0}}
$$

Now define recursively $x_{n}=x_{n-1}^{2}$. We show by induction that $F\left(x_{n}\right) \leq\left(1-\frac{\delta}{2}\right) x_{n}^{2 \alpha_{0}}$. We know this for $n=0$. Next, for $n \geq 1$, using (5.33), induction and $q \geq 2$, we have

$$
\begin{aligned}
F_{z}\left(x_{n}\right) & \leq F_{z}\left(x_{n-1}\right)^{2}+C x_{n}^{\beta / 4} \leq\left(1-\frac{\delta}{2}\right)^{2} x_{n}^{2 \alpha_{0}}+x_{n}^{2 \alpha_{0}} \cdot C x_{0}^{\beta / 4-2 \alpha_{0}} \\
& \leq\left[\left(1-\frac{\delta}{2}\right)^{2}+C x_{0}^{\beta / 8}\right] x_{n}^{2 \alpha_{0}} \leq\left(1-\frac{\delta}{2}\right) x_{n}^{2 \alpha_{0}}
\end{aligned}
$$

where we used $x_{n} \leq x_{0}, 2 \alpha_{0} \in(0, \beta / 8]$ and $x_{0} \leq\left(\frac{\delta}{4 C}\right)^{8 / \beta}$. Hence, if $x \in\left(0, x_{0}\right]$, so that $x \in\left(x_{n+1}, x_{n}\right]$ for some $n$, using that $F_{z}$ is monotone increasing, we get

$$
F_{z}(x) \leq F_{z}\left(x_{n}\right) \leq\left(1-\frac{\delta}{2}\right) x_{n+1}^{\alpha_{0}} \leq\left(1-\frac{\delta}{2}\right) x^{\alpha_{0}} \leq x^{\alpha_{0}} .
$$


This proves a first power decay which we now improve. Suppose we have

$$
F_{z}(x) \leq c x^{\alpha}
$$

for some $c>0$ and all $x \in\left(0, x_{0}\right]$. Taking $y=x^{\frac{1}{3} \frac{5}{1+5}}$ in Lemma 5.5, we get

$$
\begin{aligned}
F_{z}(x) & \leq F_{z}\left(x^{\frac{3+\varsigma}{3(1+\varsigma)}}\right)^{q}+C x^{\frac{\beta}{3} \frac{\varsigma}{1+\varsigma}} F_{z}\left(\frac{4 Q c_{2}}{c_{1}^{2}} x^{\frac{1}{3} \frac{\varsigma}{1+\varsigma}}\right)^{q}+C x^{\frac{1}{3} \frac{\varsigma^{2}}{1+\varsigma}} \\
& \leq c x^{\frac{q}{3} \frac{3+\varsigma}{1+\varsigma} \alpha}+\tilde{C} x^{\frac{1}{3} \frac{\varsigma}{\varsigma+1}(\beta+q \alpha)}+C x^{\frac{1}{3} \frac{\varsigma^{2}}{1+\varsigma}} .
\end{aligned}
$$

If $q \geq 3$, then $\frac{q}{3} \frac{3+\varsigma}{1+\varsigma} \geq \frac{3+\varsigma}{1+\varsigma}>1$. Also, if $\alpha \leq \frac{\beta \varsigma}{4}$, then $\frac{\beta+q \alpha}{3} \frac{\varsigma}{1+\varsigma} \geq \frac{\beta+3 \alpha}{3} \frac{\varsigma}{1+\varsigma}>\alpha$, since $\beta \varsigma+3 \alpha \varsigma>3 \alpha+3 \alpha \varsigma$. Finally, $\frac{\varsigma}{3(1+\varsigma)} \geq \frac{1}{4} \geq \frac{\beta}{4}$, since $\varsigma \geq 3$, so $\frac{\varsigma^{2}}{3(1+\varsigma)} \geq \frac{\beta \varsigma}{4}$. We thus showed that if $q \geq 3$, then the decay power is strictly increased as long as $\alpha \leq \frac{\beta \zeta}{4}$. Iteration thus proves the theorem ${ }^{8}$ when $q \geq 3$ (because $x^{\beta \varsigma / 4} \leq x^{\beta \varsigma / 5}$ ).

The rest of the proof is devoted to the case $q=2$, where we need to improve again. Using (2.17) twice, we have

$$
\begin{aligned}
& \operatorname{Im} R_{z}^{+}\left(o_{b}\right) \geq\left|\zeta^{z}(b)\right|^{2} \sum_{b^{+}}\left|\zeta^{z}\left(b^{+}\right)\right|^{2} \sum_{b^{++}} \operatorname{Im} R_{z}^{+}\left(o_{b^{++}}\right) \\
& \quad \geq \sum_{b^{+}} \frac{c_{2}^{-2}\left|\zeta^{z}\left(b^{+}\right)\right|^{2}}{\left(\left|\alpha_{t_{b}}\right|+\left|\frac{S_{z}^{\prime}\left(L_{b}\right)}{S_{z}\left(L_{b}\right)}\right|+\sum_{b^{+}} \frac{\left|\zeta^{z}\left(b^{+}\right)\right|}{\left|S_{z}\left(L_{b^{+}}\right)\right|}+\sum_{b^{+}}\left|\frac{C_{z}\left(L_{b^{+}}\right)}{S_{z}\left(L_{b^{+}}\right)}\right|\right)^{2}} \sum_{b^{++}} \operatorname{Im} R_{z}^{+}\left(o_{b^{++}}\right) \\
& \geq \sum_{b^{+}} \frac{c_{1}^{2} c_{2}^{-2}\left|\zeta^{z}\left(b^{+}\right)\right|^{2}}{\left(c_{1}\left|\alpha_{t_{b}}\right|+\left|C_{z}\left(L_{b}\right)\right|+\sum_{b^{+}}\left|\zeta^{z}\left(b^{+}\right)\right|+\sum_{b^{+}}\left|C_{z}\left(L_{b^{+}}\right)\right|\right)^{2}} \sum_{b^{++}} \operatorname{Im} R_{z}^{+}\left(o_{b^{++}}\right)
\end{aligned}
$$

Define the events $E_{0}=\left\{\left|\alpha_{t_{b}}\right| \leq y^{-1}\right\}$,

$$
\begin{aligned}
& E_{1}=\left\{\left|\zeta^{z}\left(b^{+}\right)\right|>y^{-1} \text { for at least two } b^{+}\right\}, \\
& E_{2}=\left\{\left|\zeta^{z}\left(b_{0}^{+}\right)\right|>y^{-1} \text { for exactly one } b_{0}^{+}\right\}, \\
& E_{3}=\left\{\left|\zeta^{z}\left(b^{+}\right)\right| \leq y^{-1} \text { for all } b^{+}\right\} .
\end{aligned}
$$

Using an estimate from (5.32), we have

$$
\mathbb{P}\left(E_{1}\right) \leq \frac{Q(Q+1)}{2}\left[\mathbb{P}\left(\left|\zeta^{z}\left(b^{+}\right)\right|>y^{-1}\right)\right]^{2} \leq c_{\nu, Q}\left(\frac{2}{c_{1} y^{-1}}\right)^{2 \beta} F_{z}\left(\frac{1}{c_{1} y^{-1}}\right)^{4} .
$$

For $E_{0} \cap E_{2},\left|\zeta^{z}\left(b^{+}\right)\right| \leq y^{-1}$ for all $b^{+} \neq b_{0}^{+}$, so we have

$$
\begin{aligned}
& \frac{\left|\zeta^{z}\left(b_{0}^{+}\right)\right|}{c_{1}\left|\alpha_{t_{b}}\right|+\sum_{t_{b^{\prime}} \sim t_{b}}\left|C_{z}\left(L_{b^{\prime}}\right)\right|+\sum_{b^{+}}\left|\zeta^{z}\left(b^{+}\right)\right|} \\
& =\frac{1}{1+\frac{c_{1}\left|\alpha_{t_{b}}\right|+\sum_{t_{b^{\prime}} \sim t_{b}}\left|C_{z}\left(L_{b^{\prime}}\right)\right|+\sum_{b^{+} \neq b_{0}^{+}}\left|\zeta^{z}\left(b^{+}\right)\right|}{\left|\zeta^{z}\left(b_{0}^{+}\right)\right|}} \geq \frac{1}{Q+c_{1}+y(Q+1) c_{3}}
\end{aligned}
$$

\footnotetext{
8 The exponent of the first term is increased by $\frac{2}{1+\varsigma}$ at each step, the second one by at least $\frac{\alpha_{0}}{3(1+\varsigma)}$, as for the last one, it already has the required decay. After finitely many steps, the exponent thus reaches $\frac{\beta \zeta}{4}$.
} 
since $\frac{c_{1}\left|\alpha_{t_{b}}\right|+\sum_{t_{b^{\prime}} \sim t_{b}}\left|C_{z}\left(L_{b^{\prime}}\right)\right|+\sum_{b^{+} \neq b_{0}^{+}}\left|\zeta^{z}\left(b^{+}\right)\right|}{\left|\zeta^{z}\left(b_{0}^{+}\right)\right|} \leq \frac{c_{1} y^{-1}+(Q+1) c_{3}+Q y^{-1}}{y^{-1}}$. Assuming $y \leq 1$, the RHS is $\geq c$. Hence, for $\tilde{c}=c_{1}^{2} c_{2}^{-2} c^{2}$ we have

$$
\mathbb{P}\left(\left\{\operatorname{Im} R_{z}^{+}\left(o_{b}\right) \leq x\right\} \cap E_{0} \cap E_{2}\right) \leq \mathbb{P}\left(\tilde{c}^{2} \sum_{b_{0}^{++} \in \mathscr{N}_{b_{0}^{+}}} \operatorname{Im} R_{z}^{+}\left(o_{b_{0}^{++}}\right) \leq x\right) \leq F_{z}\left(\tilde{c}^{-2} x\right)^{2}
$$

Finally, for $E_{0} \cap E_{3}$, we use (5.31) along with $\left|\zeta^{z}(b)\right| \geq \frac{c_{1} c_{2}^{-1}}{\left(Q+c_{1}\right) y^{-1}+(Q+1) c_{3}}$. If $y$ is small, this is $\geq C y$, so we get

$\mathbb{P}\left(\left\{\operatorname{Im} R_{z}^{+}\left(o_{b}\right) \leq x\right\} \cap E_{0} \cap E_{3}\right) \leq \mathbb{P}\left(C^{2} y^{2} \sum_{b^{+}} \operatorname{Im} R_{z}^{+}\left(o_{b^{+}}\right) \leq x\right) \leq F_{z}\left(C^{-2} x y^{-2}\right)^{2}$.

Estimating $\mathbb{P}\left(E_{0}^{c}\right) \leq y^{\varsigma} M_{\varsigma}$ by Chebyshev, we thus showed that

$$
F_{z}(x) \leq C y^{2 \beta} F_{z}\left(c_{1}^{-1} y\right)^{4}+F_{z}\left(\tilde{c}^{-2} x\right)^{2}+F_{z}\left(C^{-2} x y^{-2}\right)^{2}+c^{\prime} y^{\varsigma} .
$$

Assuming we showed that $F_{z}(x) \leq c_{0} x^{\alpha}$, then choosing $y=x^{\frac{\varsigma}{3+4 \varsigma}}$, we get

$$
F_{z}(x) \leq \tilde{C} x^{\frac{2 \beta \varsigma}{3+4 \zeta}} x^{\frac{4 \alpha \varsigma}{3+4 \varsigma}}+\tilde{c} x^{2 \alpha}+\tilde{C}^{\prime} x^{\frac{3+2 \varsigma}{3+4 \varsigma}(2 \alpha)}+c^{\prime} x^{\frac{\varsigma^{2}}{3+4 \zeta}}
$$

To get $\frac{2 \beta \varsigma+4 \alpha \varsigma}{3+4 \varsigma}>\alpha$ we must have $2 \beta \varsigma+4 \alpha \varsigma>3 \alpha+4 \alpha \varsigma$, i.e. $\alpha<\frac{2 \beta \varsigma}{3}$, so $\alpha \leq \frac{\beta \varsigma}{5}$ suffices. Next, $\frac{6+4 \varsigma}{3+4 \varsigma}>1$. Finally, $\frac{\varsigma}{3+4 \varsigma} \geq \frac{1}{5} \geq \frac{\beta}{5}$ since $\varsigma \geq 3$. We thus showed the decay exponent can be strictly improved up to $\frac{\beta \varsigma}{5}$.

Proof of Theorem 1.4. Given $p \geq 1$, choose $\varsigma$ such that $p<\frac{\beta \varsigma}{5}$. By Lemma 5.4, we have $I \subseteq \sigma_{a c}^{\varepsilon}(\delta)$ for some $\delta>0$ and $\varepsilon \leq \varepsilon_{0}$. As $p \geq 1$, given $\lambda \in I$, we have by the layer-cake representation,

$\mathbb{E}\left(\left|\operatorname{Im} R_{z}^{+}\left(o_{b}\right)\right|^{-p}\right)=p \int_{0}^{\infty} t^{p-1} \mathbb{P}\left(\left|\operatorname{Im} R_{z}^{+}\left(o_{b}\right)\right|^{-1} \geq t\right) \mathrm{d} t=p \int_{0}^{\infty} t^{p-1} F_{z}\left(t^{-1}\right) \mathrm{d} t$.

By Theorem 5.3, denoting $t_{\delta}=x_{\delta}^{-1}$, we know $F_{z}\left(t^{-1}\right) \leq C_{\nu, Q} t^{-\beta \varsigma / 5}$ for all $t \geq t_{\delta}$. Hence

$\mathbb{E}\left(\left|\operatorname{Im} R_{z}^{+}\left(o_{b}\right)\right|^{-p}\right) \leq p \int_{0}^{t_{\delta}} t^{p-1} \mathrm{~d} t+p C \int_{t_{\delta}}^{\infty} t^{p-1-\beta \varsigma / 5} \mathrm{~d} t=t_{\delta}^{p}+\frac{5 p}{\beta \varsigma-5 p} \frac{C}{t_{\delta}^{\beta \varsigma / 5-p}}$.

We may assume $t_{\delta} \geq 1$ by taking a smaller $x_{\delta}$ if necessary. Since this holds for any $\lambda \in I$ and $\eta \in\left(0, \eta_{0}\right)$, we get

$$
\sup _{\lambda \in I} \sup _{\eta \in\left(0, \eta_{0}\right)} \mathbb{E}\left(\left|\operatorname{Im} R_{z}^{+}\left(o_{b}\right)\right|^{-p}\right) \leq t_{\delta}^{p}\left(1+\frac{5 p}{\beta \varsigma-5 p} C_{\nu, Q}\right) .
$$

Acknowledgements. M.I. was funded by the Labex IRMIA during part of the writing of this article. M.S. was supported by a public grant as part of the Investissement d'avenir project, reference ANR-11-LABX-0056LMH, LabEx LMH. He thanks the Université Paris Saclay for excellent working conditions, where part of this work was done. N.A. wishes to acknowledge the IUF (Institut Universitaire de France) and USIAS (University of Strasbourg Institute for Advanced Study). We thank Konstantin Pankrashkin and Simone Warzel for useful discussions. 
Open Access This article is licensed under a Creative Commons Attribution 4.0 International License, which permits use, sharing, adaptation, distribution and reproduction in any medium or format, as long as you give appropriate credit to the original author(s) and the source, provide a link to the Creative Commons licence, and indicate if changes were made. The images or other third party material in this article are included in the article's Creative Commons licence, unless indicated otherwise in a credit line to the material. If material is not included in the article's Creative Commons licence and your intended use is not permitted by statutory regulation or exceeds the permitted use, you will need to obtain permission directly from the copyright holder. To view a copy of this licence, visit http://creativecommons.org/licenses/by/4.0/.

Publisher's Note Springer Nature remains neutral with regard to jurisdictional claims in published maps and institutional affiliations.

\section{Compliance with ethical standards}

Conflict of interest The authors declare that they have no conflict of interest.

\section{A. Proofs of Some Technical Facts}

\section{A.1. General results.}

Proof of Lemma 2.1. Given $f \in L^{2}(\mathbf{T})$, define $G^{z} f:=\int_{\mathscr{T}} G^{z}(x, y) f(y) \mathrm{d} y$. We should show that $G^{z} f \in D\left(\mathscr{H}_{\mathbf{T}}\right)$ and $\left(\mathscr{H}_{\mathbf{T}}-z\right) G^{z} f=f$, so that $G^{z}=\left(\mathscr{H}_{\mathbf{T}}-z\right)^{-1}$.

Assume $f$ is continuous on $\mathscr{T}$ and supported in a ball $\Lambda \subset \mathbf{T}$. Let $x=\left(b, x_{b}\right) \in \mathscr{T}$, with $x_{b} \in\left(0, L_{b}\right)$. Fix $o, v \in \mathbf{T}$ such that $b \in B\left(\mathbf{T}_{o}^{+}\right) \cap B\left(\mathbf{T}_{v}^{-}\right)$and $\Lambda \subset \mathbf{T}_{o}^{+} \cap \mathbf{T}_{v}^{-}$. By definition,

$$
\left(G^{z} f\right)(x)=V_{z ; o}^{+}(x) \int_{\mathscr{T}_{x}^{-}} \frac{U_{z ; v}^{-}(y)}{\mathscr{W}_{v, o}^{z}(y)} f(y) \mathrm{d} y+U_{z ; v}^{-}(x) \int_{\mathscr{T}_{x}^{+}} \frac{V_{z ; o}^{+}(y)}{\mathscr{W}_{v, o}^{z}(y)} f(y) \mathrm{d} y .
$$

Hence,

$$
\left(G^{z} f\right)^{\prime}(x)=\left(V_{z ; o}^{+}\right)^{\prime}(x) \int_{\mathscr{T}_{x}^{-}} \frac{U_{z ; v}^{-}(y)}{\mathscr{W}_{v, o}^{z}(y)} f(y) \mathrm{d} y+\left(U_{z ; v}^{-}\right)^{\prime}(x) \int_{\mathscr{T}_{x}^{+}} \frac{V_{z ; o}^{+}(y)}{\mathscr{W}_{v, o}^{z}(y)} f(y) \mathrm{d} y,
$$

where the term $\frac{V_{z ; o}^{+}(x) U_{z ; v}^{-}\left(x^{-}\right)}{\mathscr{W}_{v, o}^{2}\left(x^{-}\right)} f\left(x^{-}\right)-\frac{U_{z ; v}^{-}(x) V_{z ; o}^{+}\left(x^{+}\right)}{\mathscr{W}_{v, o}^{2}\left(x^{+}\right)} f\left(x^{+}\right)$from Leibniz's rule canceled, all functions being continuous. Next,

$$
\begin{aligned}
\left(G^{z} f\right)^{\prime \prime}(x)= & \left(V_{z ; o}^{+}\right)^{\prime \prime}(x) \int_{\mathscr{T}_{x}^{-}} \frac{U_{z ; v}^{-}(y)}{\mathscr{W}_{v, o}^{z}(y)} f(y) \mathrm{d} y \\
& +\left(U_{z ; v}^{-}\right)^{\prime \prime}(x) \int_{\mathscr{T}_{x}^{+}} \frac{V_{z ; o}^{+}(y)}{\mathscr{W}_{v, o}^{z}(y)} f(y) \mathrm{d} y-f(x),
\end{aligned}
$$

where we used that $\frac{\left(V_{z ; o}^{+}\right)^{\prime}(x) U_{z ;}^{-}\left(x^{-}\right)}{\mathscr{W}_{v, o}^{z}\left(x^{-}\right)} f\left(x^{-}\right)-\frac{\left(U_{z ; v}^{-}\right)^{\prime}(x) V_{z ; o}^{+}\left(x^{+}\right)}{\mathscr{W}_{v, o}^{z}\left(x^{+}\right)} f\left(x^{+}\right)=-f(x)$. Recalling that $\psi^{\prime \prime}=(W-z) \psi$ for $\psi=V_{z ; o}^{+}, U_{z ; v}^{-}$, we get $\left(G^{z} f\right)^{\prime \prime}=(W-z) G^{z} f-f$, so $\left(\mathscr{H}_{\mathbf{T}}-z\right) G^{z} f=f$.

For the boundary conditions, note that $G^{z} f(x)=\int_{\mathbf{T}} G^{z}(x, y) f(y) \mathrm{d} y$ and $\left(G^{z} f\right)^{\prime}(x)=$ $\int_{\mathbf{T}} \partial_{x} G^{z}(x, y) f(y) \mathrm{d} y$ by (A.1). So it suffices to check that $x \mapsto G^{z}(x, y)$ satisfies the $\delta$ conditions. But this follows immediately since $V_{z ; o}^{+} \in D\left(\mathscr{H}_{\mathbf{T}_{o}^{+}}^{\max }\right)$ and $U_{z ; v}^{-} \in D\left(\mathscr{H}_{\mathbf{T}_{v}^{-}}^{\max }\right)$. 
Finally, as $H_{\mathbf{T}}$ is self-adjoint, we have by the spectral theorem $\|f\|^{2}=\|\left(\mathscr{H}_{\mathbf{T}}-\right.$ z) $G^{z} f\left\|^{2}=\int_{\mathbf{T}}|\lambda-z|^{2} \mathrm{~d} \mu_{G^{z} f} \geq|\operatorname{Im} z|^{2} \int_{\mathbf{T}} \mathrm{d} \mu_{G^{z} f}=|\operatorname{Im} z|^{2}\right\| G^{z} f \|^{2}$, i.e. $\left\|G^{z} f\right\| \leq$ $\frac{1}{\operatorname{Im} z}\|f\|$. This holds on the subspace of continuous $f$ of compact support. By the density of such functions, $G^{z}$ extends to a bounded operator on $L^{2}(\mathbf{T})$ satisfying $\left\|G^{z}\right\| \leq \frac{1}{\operatorname{Im} z}$.

We proved that $G^{z} f \in D\left(\mathscr{H}_{\mathbf{T}}\right)$ and $\left(\mathscr{H}_{\mathbf{T}}-z\right) G^{z} f=f$ assuming $f$ is continuous of compact support. For general $f \in L^{2}(\mathbf{T})$, take a sequence $\left(f_{j}\right)$ of such functions with $f_{j} \rightarrow L_{2} f$. We showed $G^{z} f_{j} \in W_{\max }^{2,2}(\mathbf{T})$ for each $j$; this space being complete, we obtain a subsequence $\left(G^{z} f_{j_{k}}\right)$ converging in $W_{\max }^{2,2}(\mathbf{T})$. The limit must be $G^{z} f$ since $G^{z} f$ is the $L^{2}$ limit of $G^{z} f_{j}$. It follows that $\left(\mathscr{H}_{\mathbf{T}}-z\right) G^{z} f=f$ a.e., and for each $x$, we have $G^{z} f(x)=\lim _{k} G^{z} f_{j_{k}}(x)$ and $\left(G^{z} f\right)^{\prime}(x)=\lim _{k}\left(G^{z} f_{j_{k}}\right)^{\prime}(x)$, so we deduce that $G^{z} f$ satisfies the boundary conditions.

Remark A.1. Fix an edge $b \in B(\mathbb{T})$ and choose $o$, $v$ with $b \in B\left(\mathbf{T}_{o}^{+}\right) \cap B\left(\mathbf{T}_{v}^{-}\right)$. Then for $x=\left(b, x_{b}\right), y=\left(b, y_{b}\right) \in \mathscr{T}$, we can also express

$$
G_{\mathbf{T}}^{z}(x, y)= \begin{cases}-\frac{\phi_{z ; b}^{-}(x) \phi_{z ; b}^{+}(y)}{R_{z}^{+}\left(o_{b}\right)+R_{z}^{-}\left(o_{b}\right)} & \text { if } y \in \mathscr{T}_{x}^{+}, \\ -\frac{\phi_{z ; b}^{-}(y) \phi_{z ; b}^{+}(x)}{R_{z}^{+}\left(o_{b}\right)+R_{z}^{-}\left(o_{b}\right)} & \text { if } y \in \mathscr{T}_{x}^{-},\end{cases}
$$

where $\phi_{z ; b}^{-}(x)=\frac{U_{z ; v}^{-}(x)}{U_{z ; v}^{-}\left(o_{b}\right)}$ and $\phi_{z ; b}^{+}(x)=\frac{V_{z ; o}^{+}(x)}{V_{z ; o}^{+}\left(o_{b}\right)}$. This follows immediately from (2.1), (2.2), since the Wronskian $\mathscr{W}_{v, o}^{z}(y)=\mathscr{W}_{v, o}^{z}\left(b, y_{b}\right)$ is constant for $y_{b} \in\left[0, L_{b}\right]$. Note that

$$
\phi_{z ; b}^{ \pm}(x)=C_{z}\left(x_{b}\right) \pm R_{z}^{ \pm}\left(o_{b}\right) S_{z}\left(x_{b}\right)
$$

for $x=\left(b, x_{b}\right) \in \mathscr{T}$. On the other hand,

$$
G_{\mathbf{T}}^{z}(y, x)=G_{\mathbf{T}}^{z}(x, y)
$$

for $x=\left(b, x_{b}\right), y=\left(b, y_{b}\right) \in \mathscr{T}$, since $x \in \mathscr{T}_{y}^{ \pm} \Longleftrightarrow y \in \mathscr{T}_{x}^{\mp}$. This implies

$$
\left\langle f_{1}, G^{z} f_{2}\right\rangle=\left\langle f_{2}, G^{z} f_{1}\right\rangle
$$

for any real-valued $f_{j}$ supported on $e(b)$. Hence, for any $f=f_{1}+\mathrm{i} f_{2}$ supported in $e$,

$$
\left\langle f, G^{z} f\right\rangle=\left\langle f_{1}, G^{z} f_{1}\right\rangle+\left\langle f_{2}, G^{z} f_{2}\right\rangle .
$$

Lemma A.1. Fix $b \in B(\mathbb{T})$ and suppose $R_{\lambda}^{ \pm}\left(o_{b}\right):=R_{\lambda+\mathrm{i} 0}^{ \pm}\left(o_{b}\right)$ exist and are not both zero. Then for any $f$ supported in $e(b)$,

$$
\operatorname{Im}\left\langle f, G^{\lambda} f\right\rangle=\frac{\operatorname{Im} R_{\lambda}^{+}\left(o_{b}\right) g_{f}^{-}(\lambda)+\operatorname{Im} R_{\lambda}^{-}\left(o_{b}\right) g_{f}^{+}(\lambda)}{\left|R_{\lambda}^{+}\left(o_{b}\right)+R_{\lambda}^{-}\left(o_{b}\right)\right|^{2}},
$$

where

$$
g_{f}^{ \pm}(\lambda)=\left|\left\langle f, \operatorname{Re} \phi_{\lambda ; b}^{ \pm}\right\rangle_{L^{2}\left[0, L_{b}\right]}\right|^{2}+\left|\left\langle f, \operatorname{Im} \phi_{\lambda ; b}^{ \pm}\right\rangle_{L^{2}\left[0, L_{b}\right]}\right|^{2}
$$

In particular, if $\operatorname{Im} R_{\lambda}^{ \pm}\left(o_{b}\right)=0$ for all $b \in B$, then $\operatorname{Im}\left\langle f, G^{\lambda} f\right\rangle=0$ for all $f \in L^{2}(\mathbf{T})$. This lemma was stated without proof in [1, Eq. (A.15)]. 
Proof. First note that it suffices to prove this for real-valued $f$. In fact, for $f=f_{1}+\mathrm{i} f_{2}$, we then use (A.4) and deduce the result, since $g_{f_{1}}^{+}(\lambda)+g_{f_{2}}^{+}(\lambda)=g_{f}^{+}(\lambda)$ as easily checked and similarly for $g_{f}^{-}(\lambda)$. So assume $f$ is real.

Since $b$ and $\lambda$ are fixed, we shall denote $R^{ \pm}:=R_{\lambda}^{ \pm}\left(o_{b}\right)$ and $\phi^{ \pm}:=\phi_{\lambda ; b}^{ \pm}$, and drop $b$ indices in $x_{b}$ and $f_{b}$ for simplicity.

We have by (A.2),

$$
\begin{aligned}
\left\langle f, G^{\lambda} f\right\rangle= & \frac{-1}{R^{+}+R^{-}} \int_{0}^{L_{b}} f(x)\left(\int_{0}^{x} \phi^{+}(x) \phi^{-}(y) f(y) \mathrm{d} y\right. \\
& \left.+\int_{x}^{L_{b}} \phi^{-}(x) \phi^{+}(y) f(y) \mathrm{d} y\right) \mathrm{d} x .
\end{aligned}
$$

But by (A.3),

$$
\begin{aligned}
& \phi^{+}(x) \phi^{-}(y)=C(x) C(y)-R^{-} C(x) S(y)+R^{+} S(x) C(y)-R^{+} R^{-} S(x) S(y), \\
& \phi^{-}(x) \phi^{+}(y)=C(x) C(y)+R^{+} C(x) S(y)-R^{-} S(x) C(y)-R^{+} R^{-} S(x) S(y) .
\end{aligned}
$$

Thus, $\phi^{-}(x) \phi^{+}(y)=\phi^{+}(x) \phi^{-}(y)+\left(R^{+}+R^{-}\right)(C(x) S(y)-S(x) C(y))$. Hence,

$$
\begin{aligned}
\left\langle f, G^{\lambda} f\right\rangle= & \frac{-1}{R^{+}+R^{-}} \int_{\left[0, L_{b}\right]^{2}} f(x) f(y) \phi^{+}(x) \phi^{-}(y) \mathrm{d} y \mathrm{~d} x \\
& -\int_{0}^{L_{b}} \int_{x}^{L_{b}} f(x) f(y)(C(x) S(y)-S(x) C(y)) \mathrm{d} y \mathrm{~d} x .
\end{aligned}
$$

Since $f$ is real-valued, we get

$$
\begin{aligned}
\operatorname{Im}\left\langle f, G^{\lambda} f\right\rangle= & \frac{\operatorname{Im}\left(R^{+}+R^{-}\right)}{\left|R^{+}+R^{-}\right|^{2}} \int_{\left[0, L_{b}\right]^{2}} f(x) f(y) \operatorname{Re}\left[\phi^{+}(x) \phi^{-}(y)\right] \mathrm{d} y \mathrm{~d} x \\
& -\frac{\operatorname{Re}\left(R^{+}+R^{-}\right)}{\left|R^{+}+R^{-}\right|^{2}} \int_{\left[0, L_{b}\right]^{2}} f(x) f(y) \operatorname{Im}\left[\phi^{+}(x) \phi^{-}(y)\right] \mathrm{d} y \mathrm{~d} x \\
= & \frac{1}{\left|R^{+}+R^{-}\right|^{2}}\left\{\operatorname{Im}\left(R^{+}+R^{-}\right)\left[\left\langle f, \operatorname{Re} \phi^{+}\right\rangle\left\langle f, \operatorname{Re} \phi^{-}\right\rangle-\left\langle f, \operatorname{Im} \phi^{+}\right\rangle\left\langle f, \operatorname{Im} \phi^{-}\right\rangle\right]\right. \\
& \left.-\operatorname{Re}\left(R^{+}+R^{-}\right)\left[\left\langle f, \operatorname{Im} \phi^{+}\right\rangle\left\langle f, \operatorname{Re} \phi^{-}\right\rangle+\left\langle f, \operatorname{Re} \phi^{+}\right\rangle\left\langle f, \operatorname{Im} \phi^{-}\right\rangle\right]\right\} .
\end{aligned}
$$

Now $\operatorname{Re} \phi^{ \pm}=C(x) \pm\left(\operatorname{Re} R^{ \pm}\right) S(x)$ and $\operatorname{Im} \phi^{ \pm}=\left( \pm \operatorname{Im} R^{ \pm}\right) S(x)$. Hence, the term in curly brackets is

$$
\begin{aligned}
\operatorname{Im} & \left(R^{+}+R^{-}\right)\left[\langle f, C\rangle+\operatorname{Re} R^{+}\langle f, S\rangle\right]\left[\langle f, C\rangle-\operatorname{Re} R^{-}\langle f, S\rangle\right] \\
& -\operatorname{Im}\left(R^{+}+R^{-}\right)\left[\operatorname{Im} R^{+}\langle f, S\rangle\right]\left[-\operatorname{Im} R^{-}\langle f, S\rangle\right] \\
& -\operatorname{Re}\left(R^{+}+R^{-}\right)\left[\operatorname{Im} R^{+}\langle f, S\rangle\right]\left[\langle f, C\rangle-\operatorname{Re} R^{-}\langle f, S\rangle\right] \\
& -\operatorname{Re}\left(R^{+}+R^{-}\right)\left[\langle f, C\rangle+\operatorname{Re} R^{+}\langle f, S\rangle\right]\left[-\operatorname{Im} R^{-}\langle f, S\rangle\right] \\
= & \langle f, C\rangle^{2} \operatorname{Im}\left(R^{+}+R^{-}\right)+\langle f, C\rangle\langle f, S\rangle\left[\left(\operatorname{Im} R^{+}+\operatorname{Im} R^{-}\right)\left(\operatorname{Re} R^{+}-\operatorname{Re} R^{-}\right)\right. \\
& \left.-\left(\operatorname{Re} R^{+}+\operatorname{Re} R^{-}\right)\left(\operatorname{Im} R^{+}-\operatorname{Im} R^{-}\right)\right] \\
& +\langle f, S\rangle^{2}\left[\left(\operatorname{Im} R^{+}+\operatorname{Im} R^{-}\right)\left(\operatorname{Im} R^{+} \operatorname{Im} R^{-}-\operatorname{Re} R^{+} \operatorname{Re} R^{-}\right)\right. \\
& \left.+\left(\operatorname{Re} R^{+}+\operatorname{Re} R^{-}\right)\left(\operatorname{Im} R^{+} \operatorname{Re} R^{-}+\operatorname{Im} R^{-} \operatorname{Re} R^{+}\right)\right]
\end{aligned}
$$




$$
\begin{aligned}
= & \langle f, C\rangle^{2} \operatorname{Im}\left(R^{+}+R^{-}\right)+2\langle f, C\rangle\langle f, S\rangle\left[\operatorname{Im} R^{-} \operatorname{Re} R^{+}-\operatorname{Im} R^{+} \operatorname{Re} R^{-}\right] \\
& +\langle f, S\rangle^{2}\left[\left(\operatorname{Im} R^{+}\right)^{2} \operatorname{Im} R^{-}+\operatorname{Im} R^{+}\left(\operatorname{Im} R^{-}\right)^{2}+\operatorname{Im} R^{-}\left(\operatorname{Re} R^{+}\right)^{2}\right. \\
& \left.+\operatorname{Im} R^{+}\left(\operatorname{Re} R^{-}\right)^{2}\right] .
\end{aligned}
$$

On the other hand,

$$
\begin{aligned}
\operatorname{Im} R^{+} g_{f}^{-}+\operatorname{Im} R^{-} g_{f}^{+}= & \operatorname{Im} R^{+}\left[\left(\langle f, C\rangle-\operatorname{Re} R^{-}\langle f, S\rangle\right)^{2}+\left(\operatorname{Im} R^{-}\right)^{2}\langle f, S\rangle^{2}\right] \\
& +\operatorname{Im} R^{-}\left[\left(\langle f, C\rangle+\operatorname{Re} R^{+}\langle f, S\rangle\right)^{2}+\left(\operatorname{Im} R^{+}\right)^{2}\langle f, S\rangle^{2}\right],
\end{aligned}
$$

which is exactly the expression at the end of (A.7). This completes the proof of (A.5).

Finally, if $\operatorname{Im} R_{\lambda}^{ \pm}\left(o_{b}\right)=0$ for all $b \in B$, then $\operatorname{Im}\left\langle f_{b}, G^{\lambda} f_{b}\right\rangle=0$ for all $f_{b}$ supported in $b$, by (A.5). The same ideas show that $\operatorname{Im}\left\langle f_{b}, G^{\lambda} f_{b^{\prime}}\right\rangle=0$ in this case. In fact, we don't need to go through all the above calculations, just note that in (A.6), we get $\operatorname{Im} \phi^{ \pm}=\left( \pm \operatorname{Im} R^{ \pm}\right) S=0$.

It follows that $\operatorname{Im}\left\langle f, G^{\lambda} f\right\rangle=0$ for all $f \in L^{2}(\mathbf{T})$.

Proof of Lemma 2.3. We may find an $L^{2}$ solution $\widetilde{U}_{z ; v}^{-}$on $\mathbf{T}_{v}^{-}$satisfying the $\delta$-conditions at vertices $w \notin\left\{o_{b}, v\right\}$, the Neumann condition at $o_{b}$ and $\widetilde{U}_{z ; v}^{-}(v)=1$. As in (2.1), we get

$$
G_{\mathbf{T}_{o_{b}}^{+}}^{z}(x, y)= \begin{cases}\frac{\widetilde{U}_{z i v}^{-}(x) V_{z ; o}^{+}(y)}{\mathscr{W}_{v, o, o}^{2}(x)} & \text { if } y \in \mathscr{T}_{x}^{+}, \\ \frac{\widetilde{U}_{z ;}^{-}(y) V_{z ; o}^{+}(x)}{\mathscr{W}_{v, o, o_{b}}^{2}(x)} & \text { if } y \in \mathscr{T}_{x}^{-},\end{cases}
$$

where $\mathscr{W}_{v, o, o_{b}}^{z}(x)=V_{z ; o}^{+}(x)\left(\widetilde{U}_{z ; v}^{-}\right)^{\prime}(x)-\left(V_{z ; o}^{+}\right)^{\prime}(x) \widetilde{U}_{z ; v}^{-}(x)$.

In particular, $\frac{1}{G_{\mathbf{T}_{o_{b}}^{+}}^{z}\left(o_{b}, o_{b}\right)}=\frac{\mathscr{W}_{v, o, o_{b}}^{z}\left(o_{b}\right)}{U_{z ; v}^{-}\left(o_{b}\right) V_{z ; o}^{+}\left(o_{b}\right)}=0-\frac{\left(V_{z ; o}\right)^{\prime}\left(o_{b}\right)}{V_{z ; o}\left(o_{b}\right)}=-R_{z}^{+}\left(o_{b}\right)$.

Similarly, we find $\widetilde{V}_{z ; o}^{+}$on $\mathbf{T}_{o}^{+}$satisfying Neumann's condition at $t_{b}$, yielding

$$
G_{\mathbf{T}_{t_{b}}^{-}}^{z}(x, y)= \begin{cases}\frac{U_{z, v}^{-}(x) \tilde{V}_{z ; o}^{+}(y)}{\mathscr{W}} & \text { if } y \in \mathscr{T}_{x}^{+}, \\ \frac{U_{z ;, t_{t}}^{-}(y)(x) V_{z ; o}^{+}(x)}{\mathscr{W}_{v, o, t_{b}}^{2}(x)} & \text { if } y \in \mathscr{T}_{x}^{-},\end{cases}
$$

so we get similarly $R_{z}^{-}\left(t_{b}\right)=\frac{-1}{G_{\mathbf{T}_{t_{b}}^{-}}^{2}\left(t_{b}, t_{b}\right)}$.

To see the Herglotz property [17], note that if $\mathscr{H}_{\mathbf{T}_{u}^{ \pm}}^{\max }$ is as in Sect. 2, then

$$
\left\langle V_{z ; u}^{+}, \mathscr{H}_{\mathbf{T}_{u}^{+}}^{\max } V_{z ; u}^{+}\right\rangle_{L^{2}\left(\mathscr{T}_{u}^{+}\right)}-\left\langle\mathscr{H}_{\mathbf{T}_{u}^{+}}^{\max } V_{z ; u}^{+}, V_{z ; u}^{+}\right\rangle_{L^{2}\left(\mathscr{T}_{u}^{+}\right)}=2 \mathrm{i} \operatorname{Im} z \cdot\left\|V_{z ; u}^{+}\right\|_{L^{2}\left(\mathscr{T}_{u}^{+}\right)}^{2} .
$$

On the other hand, the left-hand side can also be computed by integration by parts on every edge. All the boundary terms except the one at $u$ cancel thanks to the self-adjoint conditions. We thus obtain

$$
\begin{aligned}
& \left\langle V_{z ; u}^{+}, \mathscr{H}_{\mathbf{T}_{u}^{+}}^{\max } V_{z ; u}^{+}\right\rangle_{L^{2}\left(\mathscr{T}_{u}^{+}\right)}-\left\langle\mathscr{H}_{\mathbf{T}_{u}^{+}}^{\max } V_{z ; u}^{+}, V_{z ; u}^{+}\right\rangle_{L^{2}\left(\mathscr{T}_{u}^{+}\right)} \\
& =\overline{V_{z ; u}^{+}(u)}\left(V_{z ; u}^{+}\right)^{\prime}(u)-\overline{\left(V_{z ; u}^{+}\right)^{\prime}(u)} V_{z ; u}^{+}(u) .
\end{aligned}
$$


Since $V_{z ; u}^{+}(u)=1$, this reduces to $2 \mathrm{i} \operatorname{Im}\left(V_{z ; u}^{+}\right)^{\prime}(u)=2 \mathrm{i} \operatorname{Im} \frac{\left(V_{z ; o}^{+}\right)^{\prime}(u)}{V_{z ; o}^{+}(u)}$. We thus showed $\operatorname{Im} R_{z}^{+}(u)=\operatorname{Im} z\left\|V_{z ; u}^{+}\right\|_{\mathbf{T}_{u}^{+}}^{2}$, implying the result by taking $u=o_{b}$. The claim for $R_{z}^{-}$is similar: the preceding proof shows that in the twisted view, $\frac{\left(U_{z ; o}^{+}\right)^{\prime}(u)}{U_{z ; o}^{+}(u)}$ is Herglotz, and the negative sign is there to pass to the coherent view. These claims in turn show by (2.13) that $z \mapsto G^{z}(v, v)$ is Herglotz ${ }^{9}$

To show that $\frac{R_{z}^{+}\left(o_{b}\right)}{\sqrt{z}}$ is Herglotz, we use the same approach. First,

$$
\left\langle V_{z ; u}^{+}, \mathscr{H}_{\mathbf{T}_{u}^{+}}^{\max } V_{z ; u}^{+}\right\rangle_{L^{2}\left(\mathscr{T}_{u}^{+}\right)}+\left\langle\mathscr{H}_{\mathbf{T}_{u}^{+}}^{\max } V_{z ; u}^{+}, V_{z ; u}^{+}\right\rangle_{L^{2}\left(\mathscr{T}_{u}^{+}\right)}=2 \operatorname{Re} z \cdot\left\|V_{z ; u}^{+}\right\|_{L^{2}\left(\mathscr{T}_{u}^{+}\right)}^{2} .
$$

On the other hand, the left-hand side is $2 \operatorname{Re}\left\langle V_{z ; u}^{+}, \mathscr{H}_{\mathbf{T}_{u}^{+}}^{\max } V_{z ; u}^{+}\right\rangle$. Integrating by parts yields

$$
\begin{aligned}
& 2\left(\left\|\left(V_{z ; u}^{+}\right)^{\prime}\right\|_{L^{2}\left(\mathscr{T}_{u}^{+}\right)}^{2}+\left\langle V_{z ; u}^{+}, W V_{z ; u}^{+}\right\rangle_{L^{2}\left(\mathscr{T}_{u}^{+}\right)}\right. \\
& \left.+\sum_{v \in V\left(\mathbb{T}_{u}^{+}\right) \backslash\{u\}} \alpha_{v}\left|V_{z ; u}^{+}(v)\right|^{2}+\operatorname{Re} \overline{V_{z ; u}^{+}(u)}\left(V_{z ; u}^{+}\right)^{\prime}(u)\right) .
\end{aligned}
$$

As before, $V_{z ; u}^{+}(u)=1$ and $\left(V_{z ; u}^{+}\right)^{\prime}(u)=R_{z}^{+}(u)$. Hence, $\operatorname{Re} R_{z}^{+}(u)=\operatorname{Re} z\left\|V_{z ; u}^{+}\right\|_{L^{2}\left(\mathscr{T}_{u}^{+}\right)}^{2}-$ $\left\|\left(V_{z ; u}^{+}\right)^{\prime}\right\|_{L^{2}\left(\mathscr{T}_{u}^{+}\right)}^{2}-\left\langle V_{z ; u}^{+}, W V_{z ; u}^{+}\right\rangle_{L^{2}\left(\mathscr{T}_{u}^{+}\right)}-\sum_{v \in V\left(\mathbb{T}_{u}^{+}\right) \backslash\{u\}} \alpha_{v}\left|V_{z ; u}^{+}(v)\right|^{2}$.

Let $\sqrt{z}=r+$ is. It follows that

$$
\begin{aligned}
\operatorname{Im} & \left(\frac{R_{z}^{+}(u)}{\sqrt{z}}\right)=\operatorname{Im} R_{z} \operatorname{Re} \frac{1}{\sqrt{z}}+\operatorname{Im} \frac{1}{\sqrt{z}} \operatorname{Re} R_{z} \\
= & \frac{\operatorname{Im} z\left\|V_{z ; u}^{+}\right\|^{2} r-s \operatorname{Re} z\left\|V_{z ; u}^{+}\right\|^{2}+s\left(\left\|\left(V_{z ; u}^{+}\right)^{\prime}\right\|^{2}+\left\langle V_{z ; u}^{+}, W V_{z ; u}^{+}\right\rangle+\sum \alpha_{v}\left|V_{z ; u}^{+}(v)\right|^{2}\right)}{r^{2}+s^{2}} \\
= & \frac{\operatorname{Im}(z \overline{\sqrt{z}})\left\|V_{z ; u}^{+}\right\|^{2}+s\left(\left\|\left(V_{z ; u}^{+}\right)^{\prime}\right\|^{2}+\left\langle V_{z ; u}^{+}, W V_{z ; u}^{+}\right\rangle+\sum \alpha_{v}\left|V_{z ; u}^{+}(v)\right|^{2}\right)}{|z|} .
\end{aligned}
$$

Since $\operatorname{Im}(z \overline{\sqrt{z}})=|z| \operatorname{Im} \sqrt{z}$, the RHS is clearly positive if $W \geq 0$ and $\alpha_{v} \geq 0$ for all $v$.

We next prove the current relations. Since $V_{z ; o}^{+}$satisfies the $\delta$-conditions, we have $\sum_{b^{+} \in \mathscr{N}_{b}^{+}} R_{z}^{+}\left(o_{b^{+}}\right)=R_{z}^{+}\left(t_{b}\right)+\alpha_{t_{b}}$, so $\sum_{b^{+} \in \mathscr{N}_{b}^{+}} \operatorname{Im} R_{z}^{+}\left(o_{b^{+}}\right)=\operatorname{Im} R_{z}^{+}\left(t_{b}\right)$. Similarly, as $\sum_{w_{-} \in \mathscr{N}_{w}^{-}} U_{w_{-}}^{\prime}\left(L_{w_{-}}\right)+\alpha_{w} U_{w}(0)=U_{w}^{\prime}(0)$, we get $\sum_{b^{-} \in \mathscr{N}_{b}^{-}} \operatorname{Im} R_{z}^{-}\left(t_{b^{-}}\right)=\operatorname{Im} R_{z}^{-}\left(o_{b}\right)$.

Suppose $H f=z f$ and let $J_{z}\left(x_{b}\right)=\operatorname{Im}\left[\overline{f\left(x_{b}\right)} f^{\prime}\left(x_{b}\right)\right]$. Then $J_{z}^{\prime}\left(x_{b}\right)=\operatorname{Im}\left[\left|f^{\prime}\left(x_{b}\right)\right|^{2}+\right.$ $\overline{f\left(x_{b}\right)}\left[W\left(x_{b}\right) f\left(x_{b}\right)-z f\left(x_{b}\right)\right]=-\operatorname{Im} z\left|f\left(x_{b}\right)\right|^{2}$. Hence, $J\left(x_{b}\right)$ decreases on $b$, in particular, $J\left(t_{b}\right) \leq J\left(o_{b}\right)$. Thus,

$$
\left|V_{z ; v}^{+}\left(t_{b}\right)\right|^{2} \operatorname{Im} R_{z}^{+}\left(t_{b}\right)=\operatorname{Im}\left[\overline{V_{z ; v}^{+}\left(t_{b}\right)}\left(V_{z ; v}^{+}\right)^{\prime}\left(t_{b}\right)\right] \leq\left|V_{z ; v}^{+}\left(o_{b}\right)\right|^{2} \operatorname{Im} R_{z}^{+}\left(o_{b}\right)
$$

Thus, $\operatorname{Im} R_{z}^{+}\left(t_{b}\right) \leq \frac{\left|V_{z ; v}^{+}\left(o_{b}\right)\right|^{2}}{\left|V_{z ; v}^{+}\left(t_{b}\right)\right|^{2}} \operatorname{Im} R_{z}^{+}\left(o_{b}\right)=\frac{\operatorname{Im} R_{z}^{+}\left(o_{b}\right)}{\left|\zeta^{z}(b)\right|^{2}}$. This proves (2.17) for $R_{z}^{+}$. Since $\left|U_{z ; v}^{-}\left(o_{b}\right)\right|^{2} \operatorname{Im} R_{z}^{-}\left(o_{b}\right)=-\operatorname{Im}\left[\overline{U_{z ; v}^{-}\left(o_{b}\right)}\left(U_{z ; v}^{-}\right)^{\prime}\left(o_{b}\right)\right]$, the claim for $R_{z}^{-}$follows similarly.

9 Though it is known that $z \mapsto\left\langle\psi, G^{z} \psi\right\rangle$ is Herglotz for any $\psi \in L^{2}(\mathbf{T})$ by the spectral theorem, we followed this somehow roundabout argument to deduce the same holds for $z \mapsto G^{z}(v, v)$. See the Appendix of [7] for a more general result. 
Finally, if the terms are defined for $\operatorname{Im} z=0$, then equality follows from $J_{z}^{\prime}\left(x_{b}\right)=0$.

Remark A.2. The method above also shows that $z \mapsto-\frac{S_{z}^{\prime}(L)}{S_{z}(L)}$ is Herglotz.

In fact, $\operatorname{Im}\left(\frac{S_{z}^{\prime}(L)}{S_{z}(L)}\right)=\frac{1}{\left|S_{z}(L)\right|^{2}} \operatorname{Im}\left[\overline{S_{z}(L)} S_{z}^{\prime}(L)\right] \leq \frac{1}{\left|S_{z}(L)\right|^{2}} \operatorname{Im}\left[\overline{S_{z}(0)} S_{z}^{\prime}(0)\right]=0$. If the potentials are symmetric, so that $S_{z}^{\prime}(L)=C_{z}(L)$, we also get $\operatorname{Im}\left(\frac{C_{z}(L)}{S_{z}(L)}\right) \leq 0$.

Recall that $\operatorname{Im} R_{z}^{+}\left(t_{b}\right)=\sum_{b^{+} \in \mathscr{N}_{b}^{+}} \operatorname{Im} R_{z}^{+}\left(o_{b^{+}}\right) \geq 0$. Using (2.5),

$$
\operatorname{Im}\left(\frac{-1}{S_{z}\left(L_{b}\right) \zeta^{z}(b)}\right)=\operatorname{Im} R_{z}^{+}\left(t_{b}\right)-\operatorname{Im} \frac{S_{z}^{\prime}\left(L_{b}\right)}{S_{z}\left(L_{b}\right)} \geq 0 .
$$

Thus, we also get $\operatorname{Im}\left(S_{z}\left(L_{b}\right) \zeta^{z}(b)\right) \geq 0$ for any $b$.

Above we have shown that $\operatorname{Im} R_{z}^{+}(v)=\operatorname{Im} z\left\|V_{z ; v}^{+}\right\|_{L^{2}\left(\mathscr{T}_{v}^{+}\right)}^{2}$. We would like to replace the $L^{2}$ norm by a lower bound that does not depend on $z, v$ or any of the potentials. This is proved in the following lemma:

Lemma A.2. Let the potential $W=W_{b}$ be bounded, $\|W\|_{\infty}:=\sup _{b \in B}\left\|W_{b}\right\|_{L^{\infty}\left(\left[0, L_{b}\right]\right)}<$ $\infty$, and let $K \subset \mathbb{C}^{+}$be a compact set. Then there exists $C=C\left(K,\|W\|_{\infty}\right)>0$ such that for all $z \in K$ and all $v \in V(\mathbf{T})$, we have $\operatorname{Im} R_{z}^{+}(v) \geq C \operatorname{Im} z$.

Proof. We begin with the following general fact: let $M, \ell>0$. There exists $c>0$ such that for any potential $Q$ on $[0, \ell]$ with $\|Q\|_{\infty} \leq M$, and any solution of $-f^{\prime \prime}+Q f=0$ on $[0, \ell]$ with $f(0)=1$, we have $\|f\|_{L^{2}(0, \ell)}^{2} \geq c$. Indeed, suppose to the contrary there are $Q_{n}, f_{n}$ with $\left\|Q_{n}\right\|_{\infty} \leq M$ and $f_{n}$ solutions with $f_{n}(0)=1$ such that $\left\|f_{n}\right\|_{L^{2}(0, \ell)} \rightarrow 0$. Then $\left\|f_{n}^{\prime \prime}\right\|_{L^{2}} \rightarrow 0$. This implies $\left\|f_{n}\right\|_{W^{2,2}} \rightarrow 0$ (because in one dimension, $\|\cdot\|_{W^{2,2}}$ is equivalent to $\left.\|u\|_{L^{2}}+\left\|u^{\prime \prime}\right\|_{L^{2}}\right)$. But $W^{2,2}(0, \ell) \subset C^{1}[0, \ell]$, in particular this implies $\left\|f_{n}\right\|_{C^{0}[0, \ell]} \rightarrow 0$, so $f_{n}(0) \rightarrow 0$, a contradiction.

Taking $Q=W-z$ and $[0, \ell]$ the edge in $\mathbf{T}_{v}^{+}$outgoing from $v$ we find for $f=V_{z ; v}^{+}$,

$$
\left\|V_{z ; v}^{+}\right\|_{\mathbf{T}_{v}^{+}}^{2} \geq\|f\|_{L^{2}(0, \ell)}^{2} \geq c .
$$

We showed in Remark A.2 that $z \mapsto \frac{-S_{z}^{\prime}(L)}{S_{z}(L)}$ is Herglotz. We also have:

Lemma A.3. If the potentials $W$ are all non-negative, the function $z \mapsto-\frac{S_{z}^{\prime}(L)}{\sqrt{z} S_{z}(L)}$ is Herglotz.

Proof. Consider

$$
\begin{aligned}
\frac{\mathrm{d}}{\mathrm{d} x}\left(\frac{\overline{S_{z}^{\prime}(x)} S_{z}(x)}{\sqrt{\bar{z}}}-\frac{S_{z}^{\prime}(x) \overline{S_{z}(x)}}{\sqrt{z}}\right) \\
\quad=\frac{\overline{S_{z}^{\prime \prime}(x)} S_{z}(x)}{\sqrt{\bar{z}}}+\frac{\left|S_{z}^{\prime}(x)\right|^{2}}{\sqrt{\bar{z}}}-\frac{\left|S_{z}^{\prime}(x)\right|^{2}}{\sqrt{z}}-\frac{S_{z}^{\prime \prime}(x) \overline{S_{z}(x)}}{\sqrt{z}} \\
\quad=\frac{(W(x)-\bar{z})\left|S_{z}(x)\right|^{2}}{\sqrt{\bar{z}}}-2 \mathrm{i}\left|S_{z}^{\prime}(x)\right|^{2} \operatorname{Im}\left\{\frac{1}{\sqrt{z}}\right\}-\frac{(W(x)-z)\left|S_{z}(x)\right|^{2}}{\sqrt{z}}
\end{aligned}
$$




$$
=-2 \mathrm{i} W(x)\left|S_{z}(x)\right|^{2} \operatorname{Im}\left\{\frac{1}{\sqrt{z}}\right\}-2 \mathrm{i}\left|S_{z}^{\prime}(x)\right|^{2} \operatorname{Im}\left\{\frac{1}{\sqrt{z}}\right\}-(\sqrt{\bar{z}}-\sqrt{z})\left|S_{z}(x)\right|^{2} .
$$

Integrating this from 0 to $L$ we get

$$
\begin{aligned}
2 \mathrm{i} \operatorname{Im} \sqrt{z} \int_{0}^{L}\left|S_{z}(x)\right|^{2} \mathrm{~d} x-2 \mathrm{i} \operatorname{Im}\left\{\frac{1}{\sqrt{z}}\right\} \int_{0}^{L}\left(W(x)\left|S_{z}(x)\right|^{2}+\left|S_{z}^{\prime}(x)\right|^{2}\right) \mathrm{d} x \\
=\frac{\overline{S_{z}^{\prime}(L) S_{z}(L)}}{\sqrt{\bar{z}}}-\frac{S_{z}^{\prime}(L) \overline{S_{z}(L)}}{\sqrt{z}}-\frac{\overline{S_{z}^{\prime}(0)} S_{z}(0)}{\sqrt{\bar{z}}}+\frac{S_{z}^{\prime}(0) \overline{S_{z}(0)}}{\sqrt{z}} \\
=-2 \mathrm{i} \operatorname{Im}\left\{\frac{S_{z}^{\prime}(L) \overline{S_{z}(L)}}{\sqrt{z}}\right\}=2 \mathrm{i}\left|S_{z}(L)\right|^{2} \operatorname{Im}\left\{\frac{-S_{z}^{\prime}(L)}{\sqrt{z} S_{z}(L)}\right\} .
\end{aligned}
$$

As $W \geq 0$, we deduce that $\frac{-S_{z}^{\prime}(L)}{\sqrt{z} S_{z}(L)} \in \mathbb{C}^{+}$.

A.2. A criterion for AC spectrum. We recall the following fact [27, Theorem XIII.20].

Suppose $H$ is a self-adjoint operator on a Hilbert space $\mathscr{H}$, with resolvent $G^{z}$. Suppose there exists $p>1$ such that for any $\varphi$ in a dense subset of $\mathscr{H}$, we have

$$
\liminf _{\eta \downarrow 0} \int_{a}^{b}\left|\operatorname{Im}\left\langle\varphi, G^{\lambda+\mathrm{i} \eta} \varphi\right\rangle\right|^{p} \mathrm{~d} \lambda<\infty .
$$

Then $H$ has purely absolutely continuous spectrum in $(a, b)$.

This criterion also appeared later in [22] for $p=2$. In [27], lim inf $\eta_{\eta \downarrow}$ is replaced by $\sup _{0<\eta<1}$, but one sees from the proof that the above statement holds.

Theorem A.1. Suppose a Schrödinger operator $H_{\mathbf{T}}$ on a quantum tree satisfies the following: there exists $p>1$ such that for any $b \in \mathbf{T}$,

$$
\liminf _{\eta \downarrow 0} \int_{I} \frac{1}{\left(\operatorname{Im} R_{\lambda+\mathrm{i} \eta}^{+}\left(o_{b}\right)\left|S_{\lambda+\mathrm{i} \eta}\left(L_{b}\right)\right|^{2}\right)^{p}} \mathrm{~d} \lambda<\infty .
$$

Then $H_{\mathbf{T}}$ has purely absolutely continuous spectrum in $I$.

In particular, if conditions (1.10) and (Green-p) hold, then $\mathbb{P}$-a.e. operator $H_{\mathbf{T}}$ has pure AC spectrum in $I_{1}$.

Proof. Let $\varphi \in L^{2}(\mathscr{T})$ be continuous, compactly supported in a ball $\Lambda \subset \mathscr{T}$.

$$
\begin{aligned}
\int_{E_{0}}^{E_{1}}\left|\operatorname{Im}\left\langle\varphi, G^{\lambda+\mathrm{i} \eta} \varphi\right\rangle\right|^{p} \mathrm{~d} \lambda & =\int_{E_{0}}^{E_{1}}\left|\operatorname{Im} \int_{\Lambda \times \Lambda} \overline{\varphi(x)} G^{\lambda+\mathrm{i} \eta}(x, y) \varphi(y) \mathrm{d} x \mathrm{~d} y\right|^{p} \mathrm{~d} \lambda \\
& \leq\|\varphi\|_{\infty}^{2 p} \cdot|\Lambda|^{2(p-1)} \int_{E_{0}}^{E_{1}} \int_{\Lambda \times \Lambda}\left|G^{\lambda+\mathrm{i} \eta}(x, y)\right|^{p} \mathrm{~d} x \mathrm{~d} y \mathrm{~d} \lambda .
\end{aligned}
$$

Now $\int_{\Lambda \times \Lambda}\left|G^{z}(x, y)\right|^{p} \mathrm{~d} x \mathrm{~d} y=\frac{1}{4} \sum_{b, b^{\prime} \in B(\Lambda)} \int_{0}^{L_{b}} \int_{0}^{L_{b^{\prime}}}\left|G^{z}\left(x_{b}, y_{b^{\prime}}\right)\right|^{p} \mathrm{~d} x_{b} \mathrm{~d} y_{b^{\prime}}$. 
Fix $b, b^{\prime} \in B(\Lambda)$. Since for any $v \in V, y_{b^{\prime}} \mapsto G^{z}\left(v, y_{b^{\prime}}\right)$ is an eigenfunction with eigenvalue $z$, we have ${ }^{10}$

$$
G^{z}\left(v, y_{b^{\prime}}\right)=\frac{S_{z}\left(L_{b^{\prime}}-y_{b^{\prime}}\right) G^{z}\left(v, o_{b^{\prime}}\right)+S_{z}\left(y_{b^{\prime}}\right) G^{z}\left(v, t_{b^{\prime}}\right)}{S_{z}\left(L_{b^{\prime}}\right)},
$$

similarly for the first argument, so we deduce that for $\left(b, x_{b}\right),\left(b^{\prime}, y_{b^{\prime}}\right) \in \mathscr{T}, b \neq b^{\prime}$,

$$
\begin{aligned}
G^{z}\left(x_{b}, y_{b^{\prime}}\right)= & \frac{S_{z}\left(L_{b}-x_{b}\right) G^{z}\left(o_{b}, y_{b^{\prime}}\right)+S_{z}\left(x_{b}\right) G^{z}\left(t_{b}, y_{b^{\prime}}\right)}{S_{z}\left(L_{b}\right)} \\
= & \frac{S_{z}\left(L_{b}-x_{b}\right) S_{z}\left(L_{b^{\prime}}-y_{b^{\prime}}\right) G^{z}\left(o_{b}, o_{b^{\prime}}\right)+S_{z}\left(L_{b}-x_{b}\right) S_{z}\left(y_{b^{\prime}}\right) G^{z}\left(o_{b}, t_{b^{\prime}}\right)}{S_{z}\left(L_{b}\right) S_{z}\left(L_{b^{\prime}}\right)} \\
& +\frac{S_{z}\left(x_{b}\right) S_{z}\left(L_{b^{\prime}}-y_{b^{\prime}}\right) G^{z}\left(t_{b}, o_{b^{\prime}}\right)+S_{z}\left(x_{b}\right) S_{z}\left(y_{b^{\prime}}\right) G^{z}\left(t_{b}, t_{b^{\prime}}\right)}{S_{z}\left(L_{b}\right) S_{z}\left(L_{b^{\prime}}\right)}
\end{aligned}
$$

Let $b_{1}, \ldots, b_{k}$ be a path with $b_{1}=b$ and $b_{k}=b^{\prime}$. We observe that for any $b_{k+1} \in \mathscr{N}_{b_{k}}^{+}$,

$$
\begin{aligned}
\left|\zeta^{z}\left(b_{1}\right) \cdots \zeta^{z}\left(b_{k}\right)\right| & \leq\left[\sum_{\left(b_{2}^{\prime} ; b_{k+1}^{\prime}\right)}\left|\zeta^{z}\left(b_{1}\right) \cdots \zeta^{z}\left(b_{k}^{\prime}\right)\right|^{2} \operatorname{Im} R_{z}^{+}\left(o_{b_{k+1}^{\prime}}\right)\right]^{1 / 2} \frac{1}{\left|\operatorname{Im} R_{z}^{+}\left(o_{b_{k+1}}\right)\right|^{1 / 2}} \\
& \leq\left(\frac{\left|\operatorname{Im} R_{z}^{+}\left(o_{b_{1}}\right)\right|}{\left|\operatorname{Im} R_{z}^{+}\left(o_{b_{k+1}}\right)\right|}\right)^{1 / 2},
\end{aligned}
$$

where we used (2.17) repeatedly in the last step. On the other hand, by (2.13),

$$
\left|G^{z}\left(o_{b_{1}}, o_{b_{1}}\right)\right|=\frac{1}{\left|R_{z}^{+}\left(o_{b_{1}}\right)+R_{z}^{-}\left(o_{b_{1}}\right)\right|} \leq \frac{1}{\operatorname{Im} R_{z}^{+}\left(o_{b_{1}}\right)},
$$

where we used the fact that $R_{z}^{ \pm}(v)$ is Herglotz. Hence,

$$
\left|G^{z}\left(o_{b_{1}} ; t_{b_{k}}\right)\right| \leq \frac{1}{\left|\operatorname{Im} R_{z}^{+}\left(o_{b_{1}}\right) \operatorname{Im} R_{z}^{+}\left(o_{b_{k+1}}\right)\right|^{1 / 2}} .
$$

The other $G^{z}\left(v_{0} ; v_{r}\right)$ appearing in (A.11) are bounded similarly.

For $b=b^{\prime}$, the first equality in (A.11) should be modified as we don't have an eigenfunction at the point $x_{b}=y_{b}$. In fact, assuming without loss that $x_{b} \leq y_{b}$, we have

$$
\begin{aligned}
G^{z}\left(x_{b}, y_{b}\right)= & \frac{1}{S_{z}\left(L_{b}\right)}\left(\left[S_{z}\left(L_{b}\right) C_{z}\left(x_{b}\right)-C_{z}\left(L_{b}\right) S_{z}\left(x_{b}\right)\right] G^{z}\left(o_{b}, y_{b}\right)\right. \\
& \left.+S_{z}\left(x_{b}\right)\left[G^{z}\left(t_{b}, y_{b}\right)+S_{z}\left(L_{b}\right) C_{z}\left(y_{b}\right)-C_{z}\left(L_{b}\right) S_{z}\left(y_{b}\right)\right]\right) .
\end{aligned}
$$

This can be checked by explicit calculation from (A.2) and (2.13): these tell us that

$$
\begin{aligned}
G^{z}\left(o_{b}, y_{b}\right) & =G^{z}\left(o_{b}, o_{b}\right) \phi^{-}\left(o_{b}\right) \phi^{+}\left(y_{b}\right)=G^{z}\left(o_{b}, o_{b}\right) \phi^{+}\left(y_{b}\right), \\
G^{z}\left(t_{b}, y_{b}\right) & =G^{z}\left(o_{b}, o_{b}\right) \phi^{-}\left(y_{b}\right) \phi^{+}\left(L_{b}\right) \\
& =G^{z}\left(o_{b}, o_{b}\right)\left[\phi^{+}\left(y_{b}\right) \phi^{-}\left(L_{b}\right)-\left(R^{+}+R^{-}\right)\left(C_{z}\left(L_{b}\right) S_{z}\left(y_{b}\right)-S_{z}\left(L_{b}\right) C_{z}\left(y_{b}\right)\right)\right]
\end{aligned}
$$

10 This holds if the potentials $W$ are symmetric, otherwise one should replace $S_{z}\left(L_{b}-x_{b}\right)$ by $S_{z}\left(L_{b}\right) C_{z}\left(x_{b}\right)-$ $C_{z}\left(L_{b}\right) S_{z}\left(x_{b}\right)$. This does not affect the argument. 


$$
=G^{z}\left(o_{b}, o_{b}\right)\left[C_{z}\left(L_{b}\right)-R^{-} S_{z}\left(L_{b}\right)\right] \phi^{+}\left(y_{b}\right)+C_{z}\left(L_{b}\right) S_{z}\left(y_{b}\right)-S_{z}\left(L_{b}\right) C_{z}\left(y_{b}\right) .
$$

Here we passed from $\phi^{-}\left(y_{b}\right) \phi^{+}\left(L_{b}\right)$ to $\phi^{+}\left(y_{b}\right) \phi^{-}\left(L_{b}\right)$ as in Lemma A.1. Inserting this into the RHS of (A.12) gives $G^{z}\left(o_{b}, o_{b}\right) \phi^{-}\left(x_{b}\right) \phi^{+}\left(y_{b}\right)=G^{z}\left(x_{b}, y_{b}\right)$ as asserted.

In any case, for any $b \in B, \int_{0}^{L_{b}}\left|S_{z}\left(x_{b}\right)\right|^{p} \mathrm{~d} x_{b} \leq c$, uniformly in $\lambda+\mathrm{i} \eta \in\left(E_{0}, E_{1}\right)+$ $\mathrm{i}(0,1)$ (in fact, $\lambda \mapsto S_{\lambda}(x)$ is analytic). Recalling (A.10), (A.11), we have proved that

$$
\begin{aligned}
& \int_{E_{0}}^{E_{1}}\left|\operatorname{Im}\left\langle\varphi, G^{\lambda+\mathrm{i} \eta} \varphi\right\rangle\right|^{p} \mathrm{~d} \lambda \\
& \quad \leq \tilde{c}\|\varphi\|_{\infty}^{2 p} \cdot|\Lambda|^{2(p-1)} \sum_{b, b^{\prime} \in B(\Lambda)} \int_{E_{0}}^{E_{1}} \frac{\mathrm{d} \lambda}{\left|\operatorname{Im} R_{z}^{+}\left(o_{b}\right) \operatorname{Im} R_{z}^{+}\left(o_{b^{\prime \prime}}\right)\right|^{p / 2}\left|S_{z}\left(L_{b}\right) S_{z}\left(L_{b^{\prime}}\right)\right|^{p}},
\end{aligned}
$$

where $b^{\prime \prime}$ is any edge with $o_{b^{\prime \prime}}=t_{b^{\prime}}$. Applying Cauchy-Schwarz and (A.9), we see that (A.8) is satisfied for any continuous $\varphi$ of compact support. Hence, $H_{\mathbf{T}}$ has pure AC spectrum in $\left(E_{0}, E_{1}\right)$.

In particular, if (Green- $\boldsymbol{p}$ ) holds for a random tree, then by Fatou's lemma and Fubini, we have

$$
\begin{aligned}
& \mathbb{E}\left(\liminf _{\eta \downarrow 0} \int_{I_{1}} \sum_{t_{b} \sim o_{b}} \frac{1}{\left(\operatorname{Im} R_{\lambda+\mathrm{i} \eta}^{+}\left(o_{b}\right)\left|S_{\lambda+\mathrm{i} \eta}\left(L_{b}\right)\right|^{2}\right)^{p}} \mathrm{~d} \lambda\right) \\
& \leq \liminf _{\eta \downarrow 0} \int_{I_{1}} \mathbb{E}\left(\sum_{t_{b} \sim o_{b}} \frac{1}{\left(\operatorname{Im} R_{\lambda+\mathrm{i} \eta}^{+}\left(o_{b}\right)\left|S_{\lambda+\mathrm{i} \eta}\left(L_{b}\right)\right|^{2}\right)^{p}}\right) \mathrm{d} \lambda<\infty,
\end{aligned}
$$

where we applied (Green- $\boldsymbol{p}$ ) in the last step. It follows that the lim inf on the left-hand side is finite for $\mathbb{P}$-a.e. operator $H_{\mathbf{T}}$. Hence, $\mathbb{P}$-a.e. $H_{\mathbf{T}}$ has pure $\mathrm{AC}$ spectrum in $I_{1}$.

\section{B. The Uniform Contraction Estimate}

The proof of Proposition 5.2 goes by analyzing different cases. Following [20] we introduce "visible sets of vertices" $\operatorname{Vis}_{\gamma}(g, \varepsilon)$ and $\operatorname{Vis}_{\operatorname{Im}}^{i}(g, \varepsilon)$. The idea is that we seek a strict contraction for $\kappa_{*}^{(\pi)}$ by estimating some terms in the weighted sum (5.26). By controlling the visibility we ensure that estimates on $Q_{x, y}^{(\pi)}(g) \cos \alpha_{x, y}^{(\pi)}(g)$ do not become "invisible", for example by the weightings $q_{y}(g)$ or $\gamma_{x}(g)$ becoming too small (which would jeopardize an implied control over $c_{x}^{(\pi)}$ and $\kappa_{*}^{(p)}$, respectively [recall (5.11)(5.12)].

One sees that Lemmas 4 and 5 in [20] hold verbatim for quantum graphs.

We then study three cases to prove Proposition 5.2. In the first case, certain $\gamma_{x}^{(\pi)}(g)$ is very small. This implies the terms in the sum defining $\kappa_{*}^{(p)}(g)$ have different magnitudes, and the result follows from (an improved) Jensen inequality. In the second case it is assumed that all $\gamma_{x}^{(\pi)}(x)$ have essentially the same size, but certain $\operatorname{Im} g_{y}^{(\pi)}, y \in S_{*^{\prime}}$ is very small. In this case one proves that certain $c_{x}^{(\pi)}(g)$ must be small, so the result follows from [20, Lemma 5]. Finally in the third case, it is proved that if $g \notin B_{r}(H)$, then there are always $\pi, x, y$ such that $Q_{x, y}^{(\pi)}(g) \cos \alpha_{x, y}^{(\pi)}(g)$ is uniformly smaller than 
one. Assuming we are neither in the first nor the second case, one deduces that some $c_{x}^{(\pi)}(g)$ is small, concluding the proof again using [20, Lemma 5]. See [20, Sect. 4.7] for more details as to how these cases are put together to prove Proposition 5.2.

For quantum graphs, the first and second cases are the same as in [20]. Up to this point we are only using calculus and the definition of $\kappa_{*}^{(p)}(g)$ and $c_{x}(g)$. So more precisely, Propositions 4 and 5 from [20] hold without change to quantum graphs.

For the third case however, we need some effort to carry out the adaptation.

We start with the following lemma.

Lemma B.1. Given $z \in \mathbb{H}$, we have that $-\cot (\sqrt{z} L) \in \mathbb{H}$. In particular, assuming $W \equiv 0$, we have that $\zeta^{z}(b) \sin \left(\sqrt{z} L_{b}\right) \in \mathbb{H}$.

Proof. In Lemma A.3 the more general fact that $-\frac{S_{z}^{\prime}(L)}{\sqrt{z} S_{z}(L)}$ is Herglotz is proved. Specialising to $W \equiv 0$ we get that $-\cot \sqrt{z} L$ is Herglotz.

For the second claim, from (2.5) it follows that

$$
\frac{-1}{\sqrt{z} S_{z}\left(L_{b}\right) \zeta^{z}(b)}=\frac{R_{z}^{+}\left(t_{b}\right)}{\sqrt{z}}-\frac{S_{z}^{\prime}\left(L_{b}\right)}{\sqrt{z} S_{z}\left(L_{b}\right)},
$$

so by the previous part and the fact that $\frac{R_{z}^{+}\left(t_{b}\right)}{\sqrt{z}} \in \mathbb{H}$, we get that $\frac{-1}{\sqrt{z} S_{z}\left(L_{b}\right) \zeta^{z}(b)} \in \mathbb{H}$. Again, specialising to $W \equiv 0$ completes the proof.

In analogy to (2.4), saying that $\zeta^{z}(b)=C_{z}\left(L_{b}\right)+S_{z}\left(L_{b}\right) R_{z}^{+}\left(o_{b}\right)$, given $g \in \mathbb{H}^{S_{o, o^{\prime}}}$, we define $\zeta_{g}^{z}\left(*^{\prime}\right)=\zeta_{g}^{z}\left(*^{\prime}, \alpha, L\right)$ by

$$
\zeta_{g}^{z}\left(*^{\prime}\right)=\cos \sqrt{z} L+g_{*^{\prime}} \sin \sqrt{z} L
$$

Then $\zeta_{H}^{z}\left(*^{\prime}, \alpha_{*^{\prime}}, L_{*^{\prime}}\right)=\zeta_{0}^{z}\left(*, *^{\prime}\right)$ as expected. Moreover, from (5.23), one easily checks that $\zeta_{g}^{z}\left(*^{\prime}\right)=\frac{1}{-\phi_{*^{\prime}}(g) \sin \sqrt{z} L+\cos \sqrt{z} L}$, so that $\frac{-1}{\sin (\sqrt{z} L) \zeta_{g}^{z}\left(*^{\prime}\right)}=\phi_{*^{\prime}}(g)-\cot \sqrt{z} L$, which implies that $\sin (\sqrt{z} L) \zeta_{g}^{z}\left(*^{\prime}\right) \in \mathbb{H}$ by Lemma B.1.

We consider the argument of vectors related to the operator's Green function. The convention is that $\arg z \in(-\pi, \pi]$. Moreover, we denote by $d_{\mathbb{S}^{1}}(\cdot, \cdot)$ the translation invariant metric in $\mathbb{S}^{1}$ which is normalized by $d_{\mathbb{S}^{1}}(0, \pi)=\pi$.

Denote $Z_{v}^{z}=\zeta_{0}^{z}\left(v_{-}, v\right) \sin \sqrt{z} L_{v}^{0}$, the unperturbed $\zeta^{z} \sin \sqrt{z} L$ of the edge $\left(v_{-}, v\right)$. We define a quantity, related to the 'minimal angle' of this with the real axis, by

$$
\theta_{0}:=\frac{1}{10} \min \left\{d_{\mathbb{S}^{1}}\left(\arg Z_{k}^{z}, \beta\right) \mid \beta \in\{0, \pi\}, z \in I+\mathrm{i}[0,1], k \in \mathfrak{A}\right\},
$$

where we denoted $Z_{k}^{z}=Z_{v}^{z}$ if $v$ has label $k$. In view of Lemma B.1, since $I \subset \Sigma$ is chosen compact, the minimum exists and we have $\theta_{0}>0$. We also let

$$
\begin{gathered}
\varsigma_{0}=\min \left\{\operatorname{Im} H_{k}^{z}: z \in I+\mathrm{i}[0,1], k \in \mathfrak{A}\right\}, \\
\varsigma_{1}=\max \left\{\left|\Gamma_{k}^{z}\right|: z \in I+\mathrm{i}[0,1], k \in \mathfrak{A}\right\},
\end{gathered}
$$

where we denoted $H_{k}^{z}=H_{v}^{z}$ for $v$ of label $k$ and similarly for $\Gamma$. Again $\varsigma_{0}>0$. 
We shall also need the function

$$
\varepsilon_{1}=\varepsilon_{1}(r)=\min _{x \in S_{*, *^{\prime}}} \inf _{z \in I+\mathrm{i}[0,1]} \inf _{g_{x} \in \mathbb{H} \backslash B_{r}\left(H_{x}^{z}\right)}\left|g_{x}-H_{x}^{z}\right| .
$$

We may now state the main result of this "Appendix" (corresponding to case 3 in the above discussion).

Proposition B.1. Let $I \subset \Sigma$ be compact and satisfy (5.28). There is $c=c\left(\theta_{0}\right)<1$, $\varepsilon_{*}\left(\theta_{0}, \varsigma_{0}, I, \varepsilon_{D}\right)>0$ and $R:\left[0, \varepsilon_{*}\right) \rightarrow[0, \infty)$ with $\lim _{\varepsilon \rightarrow 0} R(\varepsilon)=0$ such that for all $\varepsilon \in\left[0, \varepsilon_{*}\right)$, if

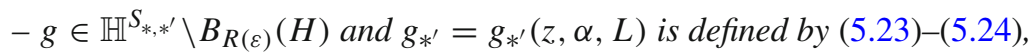

$-\left|\alpha-\alpha_{*^{\prime}}^{0}\right| \leq \varepsilon$ and $\left|L-L_{*^{\prime}}^{0}\right| \leq \varepsilon$,

$-\operatorname{Im} z \in\left[0, \eta_{*}\right]$ for some $\eta_{*}\left(\theta_{0}, \varepsilon_{D}\right)$,

then there is $\pi \in \Pi$ with

$$
Q_{x, y}^{(\pi)}(g) \leq c \quad \text { or } \quad \cos \alpha_{x, y}^{(\pi)}(g) \leq c,
$$

either for some $x, y \in S_{*^{\prime}}$ or for $x=*^{\prime}$ and all $y \in S_{*} \backslash\left\{*^{\prime}\right\}$.

Proof. First recall that for $\xi_{i} \in \mathbb{C}$

$$
d_{\mathbb{S}^{1}}\left(\arg \left(\xi_{1}\right), \arg \left(\xi_{2}\right)\right)=d_{\mathbb{S}^{1}}\left(\arg \left(\xi_{1} \bar{\xi}_{2}\right), 0\right)=d_{\mathbb{S}^{1}}\left(\arg \left(\frac{\xi_{1}}{\xi_{2}}\right), 0\right) .
$$

Also, for $\alpha, \beta, \gamma \in \mathbb{S}^{1}$, we have by the triangle inequality and translation invariance,

$$
d_{\mathbb{S}^{1}}(\alpha+\beta, \gamma) \geq d_{\mathbb{S}^{1}}(\alpha, 0)-d_{\mathbb{S}^{1}}(\beta, \gamma)
$$

Moreover, as $\alpha_{x, y}(g)=\arg \left(\left(g_{x}-H_{x}^{z}\right) \overline{\left(g_{y}-H_{y}^{z}\right)}\right)$, we have by (B.5),

$$
d_{\mathbb{S}^{1}}\left(\alpha_{x, y}(g), 0\right)=d_{\mathbb{S}^{1}}\left(\arg \left(g_{x}-H_{x}^{z}\right), \arg \left(g_{y}-H_{y}^{z}\right)\right) .
$$

By Lipschitz continuity, we may find $c_{I}$ such that for all $z \in I+\mathrm{i}[0,1]$ and $L \in$ $\bigcup_{i \in \mathfrak{A}}\left[L_{i}^{0}-1, L_{i}^{0}+1\right]$, we have

$$
\left|\sin \sqrt{z}\left(L-L_{i}^{0}\right)\right| \leq c_{I} \cdot\left|L-L_{i}^{0}\right| .
$$

We also take $c_{I}^{\prime}$ such that

$$
\left|\frac{\alpha-\alpha_{i}^{0}}{\sqrt{z}}\right| \leq c_{I}^{\prime} \cdot\left|\alpha-\alpha_{i}^{0}\right|
$$

We then choose

$$
\begin{aligned}
\varepsilon_{*} & :=\min \left(\frac{\theta_{0}}{c_{I}^{\prime}\left(1+\theta_{0}\right)} \varsigma_{0}, \frac{\theta_{0} \varepsilon_{D}}{4 c_{I} \varsigma_{1}\left(1+\theta_{0}\right)}, \frac{\theta_{0} \varepsilon_{D}}{2 c_{I}\left(1+\varsigma_{1}^{2}\right)\left(1+\theta_{0}\right)} \varsigma_{0}\right), \\
M_{I, D} & =\max \left(c_{I}^{\prime}, \frac{2 c_{I}\left(1+\varsigma_{1}^{2}\right)}{\varepsilon_{D}}\right)
\end{aligned}
$$


and define $R:\left[0, \varepsilon_{*}\right) \rightarrow[0, \infty)$ by

$$
R(\varepsilon)=\varepsilon_{1}^{-1}\left(\theta_{\varepsilon}\right), \quad \text { with } \theta_{\varepsilon}=\frac{\left(1+\theta_{0}\right)}{\theta_{0}} M_{I, D^{\varepsilon}},
$$

where $\varepsilon_{1}(r)$ is defined in (B.4). By [20, Lemma 10] or [18, Lemma 2.18], $R(\varepsilon)=$ $\frac{\theta_{\varepsilon}^{2}}{\varsigma_{0}\left(\varsigma_{0}-\theta_{\varepsilon}\right)}$ is well-defined, since $\varsigma_{0}-\theta_{\varepsilon}>0$ by the choice of $\varepsilon_{*}$.

Take $g \in \mathbb{H}^{S_{*, *^{\prime}}} \backslash B_{R(\varepsilon)}$ and $\varepsilon \in\left[0, \varepsilon_{*}\right)$. If there is $x \in S_{*, *^{\prime}}$ such that $g_{x}=H_{x}^{z}$, then $Q_{x, y}(g)=0$ by definition for all $y \in S_{*, *^{\prime}}$ and we are done. So assume that $g_{x} \neq H_{x}^{z}$ for all $x \in S_{*, *^{\prime}}$. We also assume

$$
d_{\mathbb{S}^{1}}\left(\alpha_{x, y}^{(\pi)}(g), 0\right) \leq \theta_{0} \quad \text { for all } \pi \in \Pi \text { and } x, y \in S_{*^{\prime}}
$$

since otherwise we are done. Our aim is to show $d_{\mathbb{S}^{1}}\left(\alpha_{*^{\prime}, y}^{(\pi)}(g), 0\right)>\theta_{0}$ for some $\pi \in \Pi$ and all $y \in S_{*} \backslash\left\{*^{\prime}\right\}$.

Recall that $H_{\pi(x)}^{z}=H_{x}^{z}$ for $\pi \in \Pi$ and $x \in S_{*, *^{\prime}}$. We set

$$
\tau_{*^{\prime}}^{(\pi)}:=\sum_{y \in S_{*^{\prime}}}\left(g_{y}^{(\pi)}-H_{y}^{z}\right)
$$

Recalling (B.7), our assumption (B.12) implies that

$$
\left|\tau_{*^{\prime}}^{(\pi)}\right| \geq\left|g_{x}^{(\pi)}-H_{x}^{z}\right|
$$

for all $\pi \in \Pi$ and $x \in S_{*^{\prime}}$, and

$$
d_{\mathbb{S}^{1}}\left(\arg \left(\tau_{*^{\prime}}^{(\pi)}\right), \arg \left(g_{x}^{(\pi)}-H_{x}^{z}\right)\right) \leq 2 \theta_{0}
$$

for all $\pi \in \Pi$ and $x \in S_{*, *^{\prime}}$. See [20, Lemma 9] or [18, Lemma 4.19] for a proof.

We now proceed with two claims.

Claim 1. There exists $\pi \in \Pi$ such that $\left|\tau_{*^{\prime}}^{(\pi)}\right| \geq \frac{\left(1+\theta_{0}\right)}{\theta_{0}} M_{I, D} \varepsilon$.

Proof of Claim 1. We assumed that $g \notin B_{R(\varepsilon)}$, so there is $\hat{x} \in S_{*, *^{\prime}}$ such that $\gamma_{\hat{x}}(g)>$ $R(\varepsilon)$. By the choice of $*^{\prime}$ w.r.t. (C2) there is $\pi$ such that $\pi(\hat{x}) \in S_{*^{\prime}}$. By (B.13) and the definitions (B.4) of $\varepsilon_{1}$ and $R$ above, we obtain

$$
\left|\tau_{*^{\prime}}^{\left(\pi^{-1}\right)}\right| \geq\left|g_{\pi(\hat{x})}^{\left(\pi^{-1}\right)}-H_{\pi(\hat{x})}^{z}\right|=\left|g_{\hat{x}}-H_{\hat{x}}^{z}\right| \geq \varepsilon_{1}(R(\varepsilon))=\theta_{\varepsilon}=\frac{\left(1+\theta_{0}\right)}{\theta_{0}} M_{I, D} \varepsilon .
$$

Claim 2. Denote $Z^{z}(v)=\zeta^{z}\left(v_{-}, v\right) \sin \sqrt{z} L_{v}$ and $w=\frac{\alpha-\alpha_{*^{\prime}}^{0}}{\sqrt{z}}$. Then we have

$$
\begin{aligned}
& g_{*^{\prime}}^{(\pi)}-H_{*^{\prime}}^{z}=Z_{g(\pi)}^{z}\left(*^{\prime}\right) Z_{0}^{z}\left(*^{\prime}\right)\left[( \tau _ { * ^ { \prime } } ^ { ( \pi ) } - w ) \left\{1+\cot \sqrt{z} L \cot \sqrt{z} L_{*^{\prime}}^{0}\right.\right. \\
& \left.\left.+\Gamma_{*^{\prime}}^{z} \cdot \frac{\sin \sqrt{z}\left(L-L_{*^{\prime}}^{0}\right)}{\sin \sqrt{z} L \sin \sqrt{z} L_{*^{\prime}}^{0}}\right\}+\left(\left(\Gamma_{*^{\prime}}^{z}\right)^{2}+1\right) \frac{\sin \sqrt{z}\left(L-L_{*^{\prime}}^{0}\right)}{\sin \sqrt{z} L \sin \sqrt{z} L_{*^{\prime}}^{0}}\right] .
\end{aligned}
$$


Proof of Claim 2. We have by (5.23),

$$
\begin{aligned}
g_{*^{\prime}}^{(\pi)}-H_{*^{\prime}}^{z}= & \frac{\phi_{*^{\prime}}\left(g^{(\pi)}\right) \cos \sqrt{z} L+\sin \sqrt{z} L}{-\phi_{*^{\prime}}\left(g^{(\pi)}\right) \sin \sqrt{z} L+\cos \sqrt{z} L}-\frac{\Gamma_{*^{\prime}}^{z} \cos \sqrt{z} L_{*^{\prime}}^{0}+\sin \sqrt{z} L_{*^{\prime}}^{0}}{-\Gamma_{*^{\prime}}^{z} \sin \sqrt{z} L_{*^{\prime}}^{0}+\cos \sqrt{z} L_{*^{\prime}}^{0}} \\
= & \frac{1}{\left(-\phi_{*^{\prime}}(g(\pi)) \sin \sqrt{z} L+\cos \sqrt{z} L\right)\left(-\Gamma_{*^{\prime}}^{z} \sin \sqrt{z} L_{*^{\prime}}^{0}+\cos \sqrt{z} L_{*^{\prime}}^{0}\right)} \\
& \times\left[\phi _ { * ^ { \prime } } ( g ^ { ( \pi ) } ) \left\{\cos \sqrt{z} L \cos \sqrt{z} L_{*^{\prime}}^{0}+\sin \sqrt{z} L \sin \sqrt{z} L_{*^{\prime}}^{0}\right.\right. \\
& \left.+\Gamma_{*^{\prime}}^{z}\left(\sin \sqrt{z} L \cos \sqrt{z} L_{*^{\prime}}^{0}-\cos \sqrt{z} L \sin \sqrt{z} L_{*^{\prime}}^{0}\right)\right\} \\
& -\Gamma_{*^{\prime}}^{z}\left(\cos \sqrt{z} L \cos \sqrt{z} L_{*^{\prime}}^{0}+\sin \sqrt{z} L \sin \sqrt{z} L_{*^{\prime}}^{0}\right) \\
& \left.+\sin \sqrt{z} L \cos \sqrt{z} L_{*^{\prime}}^{0}-\cos \sqrt{z} L \sin \sqrt{z} L_{*^{\prime}}^{0}\right] \\
= & \frac{\left(\phi_{*^{\prime}}\left(g^{(\pi)}\right)-\Gamma_{*^{\prime}}^{z}\right) \cos \sqrt{z}\left(L-L_{*^{\prime}}^{0}\right)+\left(\phi_{*^{\prime}}(g(\pi)) \Gamma_{*^{\prime}}^{z}+1\right) \sin \sqrt{z}\left(L-L_{*^{\prime}}^{0}\right)}{\left(-\phi_{*^{\prime}}\left(g^{(\pi)}\right) \sin \sqrt{z} L+\cos \sqrt{z} L\right)\left(-\Gamma_{*^{\prime}}^{z} \sin \sqrt{z} L_{*^{\prime}}^{0}+\cos \sqrt{z} L_{*^{\prime}}^{0}\right)} .
\end{aligned}
$$

But by reverse Möbius transformation, $\phi_{*^{\prime}}\left(g^{(\pi)}\right)=\frac{g_{*^{\prime}}^{(\pi)} \cos \sqrt{z} L-\sin \sqrt{z} L}{g_{*^{\prime}}^{(\pi)} \sin \sqrt{z} L+\cos \sqrt{z} L}$. Hence,

$$
\begin{aligned}
-\phi_{*^{\prime}}\left(g^{(\pi)}\right) \sin \sqrt{z} L+\cos \sqrt{z} L & =\frac{\sin ^{2} \sqrt{z} L-g_{*^{\prime}}^{(\pi)} \sin \sqrt{z} L \cos \sqrt{z} L}{g_{*^{\prime}}^{(\pi)} \sin \sqrt{z} L+\cos \sqrt{z} L}+\cos \sqrt{z} L \\
& =\frac{1}{g_{*^{\prime}}^{(\pi)} \sin \sqrt{z} L+\cos \sqrt{z} L}=\frac{1}{\zeta_{g^{(\pi)}}^{z}\left(*^{\prime}\right)}
\end{aligned}
$$

by definition (B.1). Thus,

$$
\begin{aligned}
g_{*^{\prime}}^{(\pi)}-H_{*^{\prime}}^{z}= & \zeta_{g^{(\pi)}}^{z}\left(*^{\prime}\right) \zeta_{0}^{z}\left(*, *^{\prime}\right)\left[( \phi _ { * ^ { \prime } } ( g ^ { ( \pi ) } ) - \Gamma _ { * ^ { \prime } } ^ { z } ) \left\{\cos \sqrt{z}\left(L-L_{*^{\prime}}^{0}\right)\right.\right. \\
& \left.+\Gamma_{*^{\prime}}^{z} \sin \sqrt{z}\left(L-L_{*^{\prime}}^{0}\right)\right\} \\
& \left.+\left(\left(\Gamma_{*^{\prime}}^{z}\right)^{2}+1\right) \sin \sqrt{z}\left(L-L_{*^{\prime}}^{0}\right)\right] .
\end{aligned}
$$

Next, $\phi_{*^{\prime}}\left(g^{(\pi)}\right)=\sum_{x \in S_{*^{\prime}}} g_{x}^{(\pi)}-\frac{\alpha}{\sqrt{z}}$, so $\left(\phi_{*^{\prime}}\left(g^{(\pi)}\right)-\Gamma_{*^{\prime}}^{z}\right)=\tau_{*^{\prime}}^{(\pi)}-w$. Claim 2 follows.

Conclusion: By Claim 2, using $\arg (\xi \zeta)=\arg (\xi)+\arg (\zeta)$ and $\arg (\xi+\zeta)=\arg (\xi)+$ $\arg (1+\zeta / \xi)$, we get

$$
\begin{aligned}
\arg \left(g_{*^{\prime}}^{(\pi)}-H_{*^{\prime}}^{z}\right)= & \arg \left(Z_{g^{(\pi)}}^{z}\left(*^{\prime}\right) Z_{0}^{z}\left(*^{\prime}\right)\right)+\arg \left[( 1 - \frac { w } { \tau _ { * ^ { \prime } } ^ { ( \pi ) } } ) \left\{1+\cot \sqrt{z} L \cot \sqrt{z} L_{*^{\prime}}^{0}\right.\right. \\
& \left.\left.+\Gamma_{*^{\prime}}^{z} \cdot \frac{\sin \sqrt{z}\left(L-L_{*^{\prime}}^{0}\right)}{\sin \sqrt{z} L \sin \sqrt{z} L_{*^{\prime}}^{0}}\right\}+\frac{\left(\Gamma_{*^{\prime}}^{z}\right)^{2}+1}{\tau_{*^{\prime}}^{(\pi)}} \cdot \frac{\sin \sqrt{z}\left(L-L_{*^{\prime}}^{0}\right)}{\sin \sqrt{z} L \sin \sqrt{z} L_{*^{\prime}}^{0}}\right] \\
& +\arg \left(\tau_{*^{\prime}}^{(\pi)}\right)
\end{aligned}
$$


We thus get for the permutation $\pi \in \Pi$ taken from Claim 1 and all $y \in S_{*} \backslash\left\{*^{\prime}\right\}$

$$
\begin{aligned}
d_{\mathbb{S}^{1}}\left(\alpha_{*^{\prime}, y}^{(\pi)}(g), 0\right)= & d_{\mathbb{S}^{1}}\left(\arg \left(g_{*^{\prime}}^{(\pi)}-H_{*^{\prime}}^{z}\right), \arg \left(g_{y}^{(\pi)}-H_{y}^{z}\right)\right) \\
\geq & d_{\mathbb{S}^{1}}\left(\arg \left(Z_{g^{(\pi)}}^{z}\left(*^{\prime}\right) Z_{0}^{z}\left(*^{\prime}\right)\right), 0\right)-d_{\mathbb{S}^{1}}\left(\operatorname { a r g } \left[1-\frac{w}{\tau_{*^{\prime}}^{(\pi)}}\right.\right. \\
& +\left(1-\frac{w}{\tau_{*^{\prime}}^{(\pi)}}\right) \Gamma_{*^{\prime}}^{z} \cdot \frac{\sin \sqrt{z}\left(L-L_{*^{\prime}}^{0}\right)}{\sin \sqrt{z} L \sin \sqrt{z} L_{*^{\prime}}^{0}} \\
& +\frac{\left(\Gamma_{*^{\prime}}^{z}\right)^{2}+1}{\tau_{*^{\prime}}^{(\pi)}} \cdot \frac{\sin \sqrt{z}\left(L-L_{*^{\prime}}^{0}\right)}{\sin \sqrt{z} L \sin \sqrt{z} L_{*^{\prime}}^{0}} \\
& \left.\left.+\left(1-\frac{w}{\tau_{*^{\prime}}^{(\pi)}}\right) \cot \sqrt{z} L \cot \sqrt{z} L_{*^{\prime}}^{0}\right], 0\right) \\
& -d_{\mathbb{S}^{1}}\left(\arg \left(\tau_{*^{\prime}}^{(\pi)}\right), \arg \left(g_{y}^{(\pi)}-H_{y}^{z}\right)\right)
\end{aligned}
$$

where we used (B.6) twice. To bound the first distance, note that we have $Z_{0}^{z}\left(*^{\prime}\right) \in \mathbb{H}$ and $d_{\mathbb{S}^{1}}\left(\arg Z_{0}^{z}\left(*^{\prime}\right), \beta\right) \geq 10 \theta_{0}$ for $\beta \in\{0, \pi\}$, by the definition (B.2) of $\theta_{0}$. Moreover, since $Z_{g^{(\pi)}}^{z}\left(*^{\prime}\right) \in \mathbb{H}$ by the argument after (B.1), we have $d_{\mathbb{S}^{1}}\left(\arg \left(Z_{g(\pi)}^{z}\left(*^{\prime}\right)\right), \beta\right)>0$ for $\beta \in\{0, \pi\}$. Hence, $d_{\mathbb{S} 1}\left(\arg \left(Z_{0}^{z}\left(*^{\prime}\right) Z_{g(\pi)}^{z}\left(*^{\prime}\right)\right), 0\right)>10 \theta_{0}$. The last distance can be estimated by (B.14).

For the second distance we begin by noticing $\left|\frac{w}{\tau_{*^{\prime}}^{(\pi)}}\right| \leq \frac{\theta_{0}}{1+\theta_{0}}$. In fact, $|w| \leq c_{I}^{\prime} \varepsilon$ by (B.9), so this follows from Claim 1 and (B.11). Also, using (B.8),

$$
\left|\frac{\left(\Gamma_{*^{\prime}}^{z}\right)^{2}+1}{\tau_{*^{\prime}}^{(\pi)}} \cdot \frac{\sin \left(\sqrt{z}\left(L-L_{*^{\prime}}^{0}\right)\right)}{\sin (\sqrt{z} L) \sin \left(\sqrt{z} L_{*^{\prime}}^{0}\right)}\right| \leq \frac{\left(\varsigma_{1}^{2}+1\right) \theta_{0}}{M_{I, D} \varepsilon\left(1+\theta_{0}\right)} \frac{c_{I} \varepsilon}{\varepsilon_{D}}
$$

and

$$
\left|\left(1-\frac{w}{\tau_{*^{\prime}}^{(\pi)}}\right) \Gamma_{*^{\prime}}^{z} \cdot \frac{\sin \left(\sqrt{z}\left(L-L_{*^{\prime}}^{0}\right)\right)}{\sin (\sqrt{z} L) \sin \left(\sqrt{z} L_{*^{\prime}}^{0}\right)}\right| \leq 2 \varsigma_{1} \frac{c_{I} \varepsilon}{\varepsilon_{D}} .
$$

Now as noted in $[20$, Lemma 8$]$, for any $\zeta, \xi \in \mathbb{C},|\zeta|<1$, we have

$$
d_{\mathbb{S}^{1}}(\arg (1+\xi+\zeta), 0) \leq \begin{cases}\frac{|\zeta|}{1-|\zeta|} & \text { if } \xi=0 \\ d_{\mathbb{S}^{1}}(\arg \xi, 0)+\frac{|\zeta|}{1-|\zeta|} & \text { if } \xi \neq 0\end{cases}
$$

We use this with $\xi=\left(1-\frac{w}{\tau_{*^{\prime}}^{(\pi)}}\right) \cot (\sqrt{z} L) \cot \left(\sqrt{z} L_{*^{\prime}}^{0}\right)$ and

$$
\begin{aligned}
\zeta= & \frac{-w}{\tau_{*^{\prime}}^{(\pi)}}+\left(1-\frac{w}{\tau_{*^{\prime}}^{(\pi)}}\right) \Gamma_{*^{\prime}}^{z} \cdot \frac{\sin \left(\sqrt{z}\left(L-L_{*^{\prime}}^{0}\right)\right)}{\sin (\sqrt{z} L) \sin \left(\sqrt{z} L_{*^{\prime}}^{0}\right)} \\
& +\frac{\left(\Gamma_{*^{\prime}}^{z}\right)^{2}+1}{\tau_{*^{\prime}}} \cdot \frac{\sin \left(\sqrt{z}\left(L-L_{*^{\prime}}^{0}\right)\right)}{\sin (\sqrt{z} L) \sin \left(\sqrt{z} L_{*^{\prime}}^{0}\right)} .
\end{aligned}
$$


From the estimates above,

$$
\begin{aligned}
|\zeta| & \leq \frac{\theta_{0}}{1+\theta_{0}}+\frac{c_{I}}{\varepsilon_{D}} \frac{\left(\varsigma_{1}^{2}+1\right) \theta_{0}}{M_{I, D}\left(1+\theta_{0}\right)}+\frac{2 c_{I} \varepsilon \varsigma_{1}}{\varepsilon_{D}} \\
& \leq \frac{\theta_{0}}{1+\theta_{0}}+\frac{\theta_{0}}{2\left(1+\theta_{0}\right)}+\frac{\theta_{0}}{2\left(1+\theta_{0}\right)} \leq \frac{3 \theta_{0}}{1+3 \theta_{0}},
\end{aligned}
$$

using (B.10), (B.11). Here, we used $\theta_{0} \leq \frac{1}{3}$ (which holds since $\theta_{0} \leq \frac{\pi}{10}$ ).

Applying (B.15) thus yields

$$
\begin{aligned}
& d_{\mathbb{S}^{1}}\left(\alpha_{*^{\prime}, y}^{(\pi)}(h), 0\right)>10 \theta_{0}-3 \theta_{0}-2 \theta_{0} \\
& \quad-d_{\mathbb{S}^{1}}\left(\arg \left[\left(1-\frac{w}{\tau_{*^{\prime}}^{(\pi)}}\right) \cot \sqrt{z} L \cot \sqrt{z} L_{*^{\prime}}^{0}\right], 0\right) \\
& \geq 5 \theta_{0}-d_{\mathbb{S}^{1}}\left(\arg \left(1-\frac{w}{\tau_{*^{\prime}}^{(\pi)}}\right), 0\right)-d_{\mathbb{S}^{1}}\left(\arg \left[\cot \sqrt{z} L \cot \sqrt{z} L_{*^{\prime}}^{0}\right], 0\right)
\end{aligned}
$$

The first distance is controlled again by (B.15) with $\xi=0$. For the second distance, we observe that $\operatorname{Re} z$ is not a Dirichlet value for $L_{*^{\prime}}^{0}, L$ by assumption (5.28), so the argument tends to 0 as $\operatorname{Im} z \downarrow 0$. So for $\operatorname{Im} z$ small enough, the last distance is $\leq \theta_{0}$. This yields

$$
d_{\mathbb{S}^{1}}\left(\alpha_{*^{\prime}, y}^{(\pi)}(g), 0\right)>5 \theta_{0}-\theta_{0}-\theta_{0}>\theta_{0} .
$$

The assertion follows by letting $c:=\cos \theta_{0}$.

\section{References}

1. Aizenman, M., Sims, R., Warzel, S.: Absolutely continuous spectra of quantum tree graphs with weak disorder. Commun. Math. Phys. 264, 371-389 (2006)

2. Aizenman, M., Warzel, S.: Absolutely continuous spectrum implies ballistic transport for quantum particles in a random potential on tree graphs. J. Math. Phys. 53, 095205 (2012)

3. Anantharaman, N., Sabri, M.: Quantum ergodicity on graphs: from spectral to spatial delocalization. Anna. Math. 189, 753-835 (2019)

4. Anantharaman, N., Sabri, M.: Quantum ergodicity for the Anderson model on regular graphs. J. Math. Phys. 58, 091901-01-091901-11 (2017)

5. Anantharaman, N., Sabri, M.: Recent results of quantum ergodicity on graphs and further investigation. Ann. Fac. Sci. Toulouse Math. 28, 559-592 (2019)

6. Anantharaman, N., Ingremeau, M., Sabri, M., Winn, B.: Quantum ergodicity for expanding quantum graphs in the regime of spectral delocalization. arXiv:2102.04169

7. Anantharaman, N., Ingremeau, M., Sabri, M., Winn, B.: Empirical spectral measures of quantum graphs in the Benjamini-Schramm limit. arXiv:2008.05709

8. Anderson, P.W.: Absence of diffusion in certain random lattices. Phys. Rev. 109, 1492-1505 (1958)

9. Avni, N., Breuer, J., Simon, B.: Periodic Jacobi matrices on trees. Adv. Math. 370, 107241 (2020)

10. Bordenave, C., Sen, A., Virág, B.: Mean quantum percolation. J. Eur. Math. Soc. 19, 3679-3707 (2017)

11. Brüning, J., Geyler, V., Pankrashkin, K.: Spectra of self-adjoint extensions and applications to solvable Schrödinger operators. Rev. Math. Phys. 20, 1-70 (2008)

12. Carlson, R.: Hill's equation for a homogeneous tree. Electron. J. Differ. Equ. 23, 1-30 (1997)

13. Carlson, R.: Quantum Cayley graphs for free groups. J. Spectr. Theory 7, 907-937 (2017)

14. Donoghue, W.F.: Monotone Matrix Functions and Analytic Continuation, Die Grundlehren der mathematischen Wissenschaften 207. Springer, Berlin (1974)

15. Exner, P.: A duality between Schrödinger operators on graphs and certain Jacobi matrices. Ann. 1'I.H.P. Sect. A 66, 359-371 (1997) 
16. Froese, R., Hasler, D., Spitzer, W.: Absolutely continuous spectrum for the Anderson model on a tree: a geometric proof of Klein's theorem. Commun. Math. Phys. 269, 239-257 (2007)

17. Hislop, P.D., Post, O.: Anderson localization for radial tree-like random quantum graphs. Waves Random Complex Media 19, 216-261 (2009)

18. Keller, M.: On the spectral theory of operators on trees. Ph.D. Thesis, 2010, Friedrich Schiller University Jena. arXiv:1101.2975

19. Keller, M., Lenz, D., Warzel, S.: On the spectral theory of trees with finite cone type. Israel J. Math. 194, 107-135 (2013)

20. Keller, M., Lenz, D., Warzel, S.: Absolutely continuous spectrum for random operators on trees of finite cone type. J. Anal. Math. 118, 363-396 (2012)

21. Kesten, H.: Symmetric random walks on groups. Trans. Am. Math. Soc. 92, 336-354 (1959)

22. Klein, A.: Extended states in the Anderson model on the Bethe lattice. Adv. Math. 133, 163-184 (1998)

23. Kuchment, P.: Quantum graphs: I. Some basic structures. Waves Random media 14, S107-128 (2004)

24. Lang, S.: Algebra, Revised Third Edition, GTM 211. Springer, Berlin (2002)

25. Ortner, R., Woess, W.: Non-backtracking random walks and cogrowth of graphs. Canad. J. Math. 59, 828-844 (2007)

26. Pöschel, J., Trubowitz, E.: Inverse Spectral Theory. Academic Press, London (1987)

27. Reed, M., Simon, B.: Methods of Modern Mathematical Physics. Vol 4.: Analysis of Operators. Academic Press, London (1978)

28. Rohleder, J., Seifert, C.: Absolutely continuous spectrum for laplacians on radial metric trees and periodicity. Integr. Equ. Oper. Theory 89, 439-453 (2017)

29. Simon, B.: Basic Complex Analysis. A Comprehensive Course in Analysis Part 2A, AMS (2015)

30. Simon, B.: Schrödinger operators in the twenty-first century. In: Fokas, A., Grigoryan, A., Kibble, T., Zegarlinski, B. (eds.) Mathematical Physics 2000, pp. 283-288. Imperial College Press, London (2000)

31. Solomyak, M.: On the spectrum of the Laplacian on regular metric trees. Waves Random Media 14, S155-S171 (2004)

32. Teschl, G.: Mathematical Methods in Quantum Mechanics. With Applications to Schrödinger Operators, Second Edition, GSM 157, AMS (2014)

Communicated by J. Marklof 